\title{
THE ROLE OF APOPTOSIS DURING INFLAMMATION RESOLUTION IN LYME ARTHRITIS
}

\author{
A Dissertation \\ Presented to \\ The Faculty of the Graduate School \\ at the \\ University of Missouri \\ In Partial Fulfillment \\ of the Requirements for the Degree
}

Doctor of Philosophy

in Microbiology

\author{
By \\ KINSEY ALYSS HILLIARD \\ Dr. Charles Robert Brown, Dissertation Advisor
}

May 2019 
The undersigned, appointed by the dean of the Graduate School, have examined the dissertation entitled:

\section{THE ROLE OF APOPTOSIS DURING INFLAMMATION RESOLUTION IN} LYME ARTHRITIS

Presented by Kinsey A. Hilliard,

a candidate for the degree of doctor of philosophy, and hereby certify that, in their opinion, is worthy of acceptance,

Certified

by

Charles R. Brown

Professor of Veterinary Pathobiology

Accepted

by

Jerod A. Skyberg

Assistant Professor of Veterinary Pathobiology

Accepted

by

Guoquan Q. Zhang

Associate Professor of Veterinary Pathobiology

Accepted

by

Mark A. Daniels

Associate Professor of Molecular Microbiology and Immunology

Accepted

by

Bumsuk Hahm

Associate Professor of Molecular Microbiology and Immunology 


\section{Dedication}

To Mom and Dad, thank you for supporting me and helping me find my own path. And to Aaron, thanks for going on this journey with me. 


\section{Acknowledgements}

I would first like to thank my parents for always encouraging me to do what makes me happy and to follow my dreams. I would not be the person or scientist I am today without your love and support. I also would like to thank my Blackburn family for believing in me and showing me the path to graduate school. I can honestly say I would have never considered this career path without your guidance, and I am forever thankful you saw my potential and gave me a push in the right direction.

A huge thank you to Aaron is also needed. I would not have made it through this journey without your love and support. Thank you for helping me get through the stressful times and always being there for me. Thanks for putting up with my crazy ideas, costumes, and obsessions and letting me be my nerdy self. We have a long way to go in life, but I am forever grateful I get to go through it with you. A big thank you is in order for my dog, Korkie, who is my number one fan. Thank you for always putting a smile on my face and being the best dog I could ever ask for.

I also owe a thank you to my fellow graduate students, especially those in Connaway Hall. Thank you so much for your friendship and support. Whether it was discussing experiments, sharing techniques and reagents, or giving mental breaks and pep talks when I needed them, I am glad I was able to go through this journey with such a supportive group of friends. Thanks for making graduate 
school more bearable. I would also like to thank my committee members and other faculty in MPT for their guidance and support throughout my graduate career. I am lucky to have been surrounded by great minds and such supportive scientists to give me the confidence to be proud of my research and my capabilities.

Last but not least, I would like to thank my mentor, Dr. Charles Brown, for all of your guidance and support during my time here at Mizzou. I am glad you taught me to "think like a scientist" and what it really means to do research. Thanks for giving me the confidence to "run with the big dogs" and to think of myself as a great scientist, even though at times I was not always confident in my own abilities. While we might not agree on the best Led Zeppelin album, I can honestly say I am a better scientist and person since I joined your lab. Thanks for believing in me and giving me the chance to make you famous. 


\section{Table of Contents}

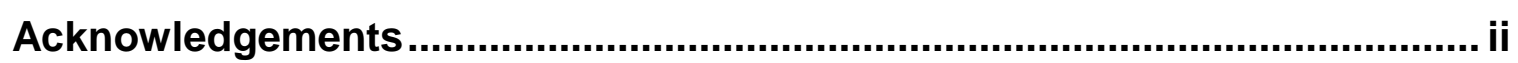

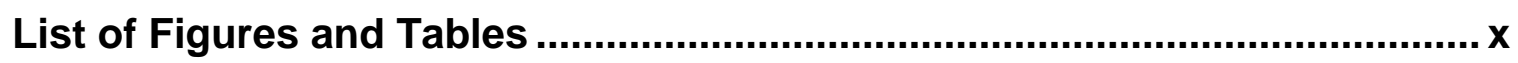

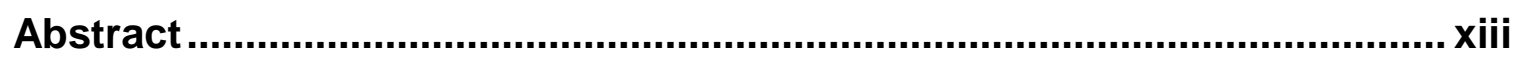

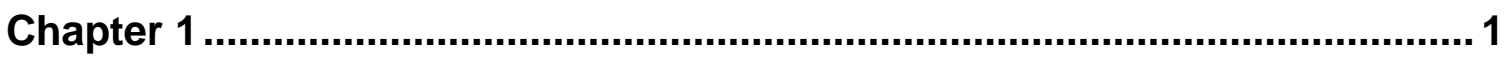

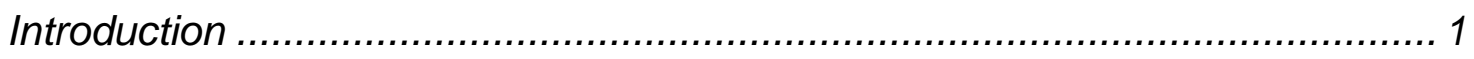

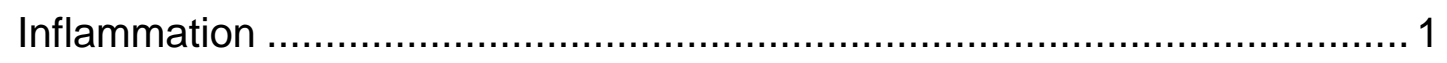

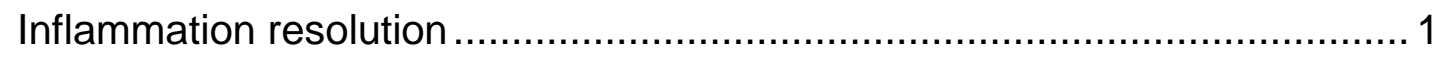

Non-resolving inflammation................................................................. 2

Apoptosis and other forms of cell death ................................................ 3

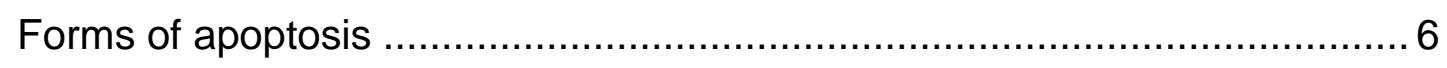

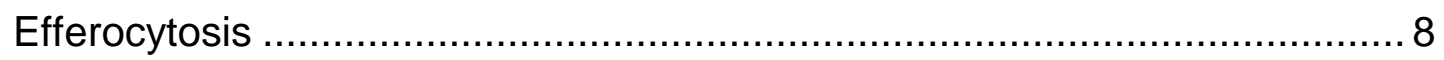

Apoptosis during bacterial infection ..................................................... 11

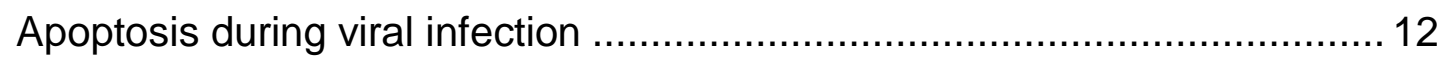

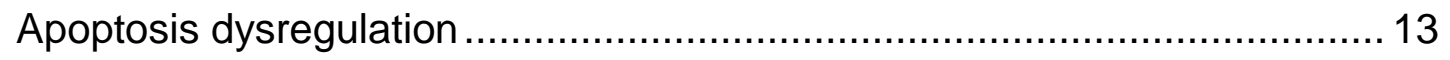

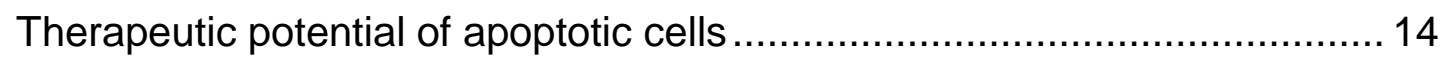

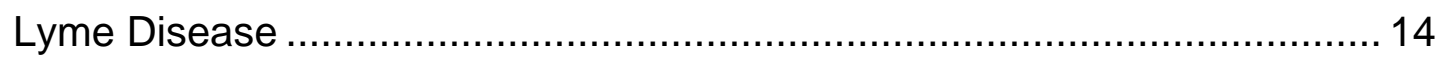

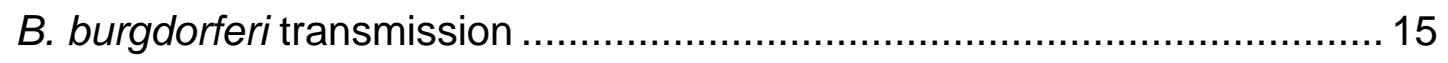

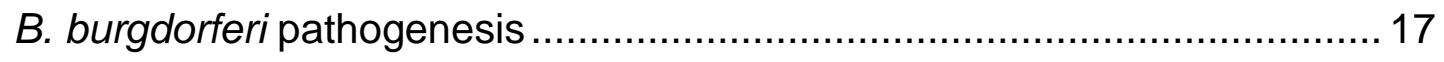

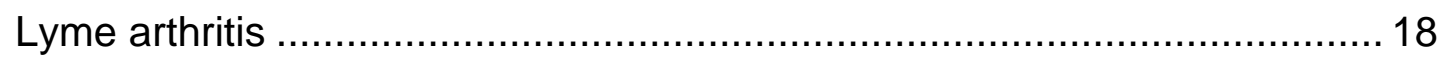

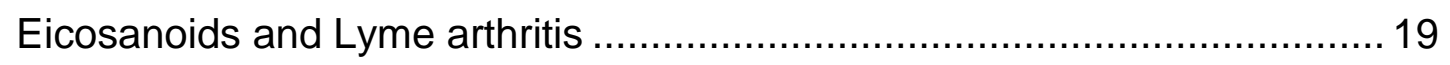

Purpose and experimental approach ................................................ 20 


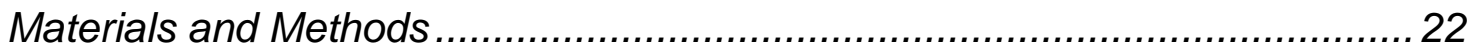

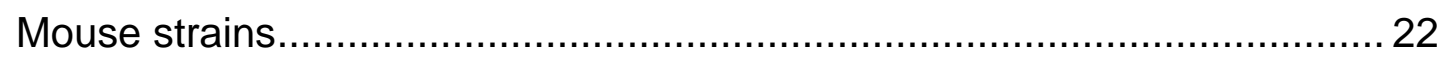

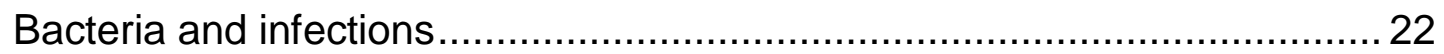

Assessment of arthritis pathology ……............................................... 23

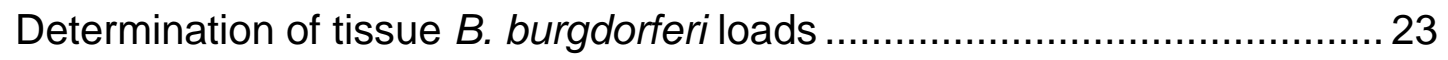

Isolation of bone marrow-derived macrophages ...................................... 24

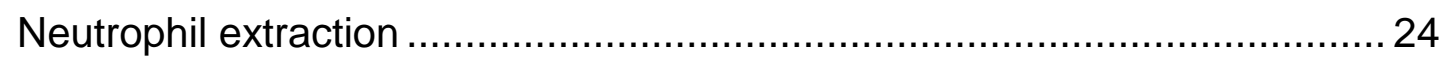

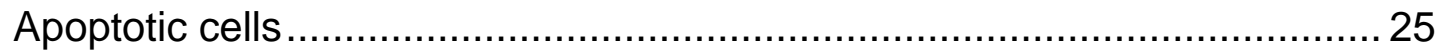

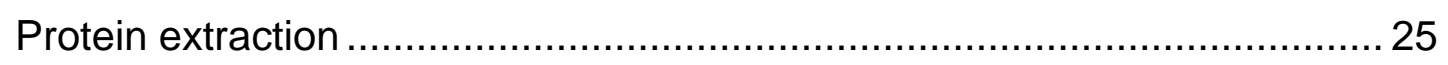

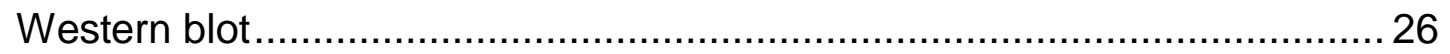

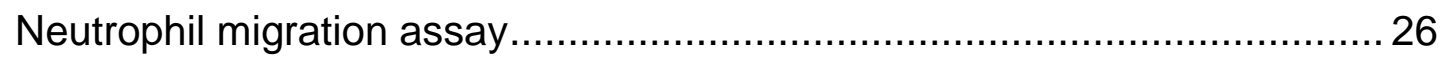

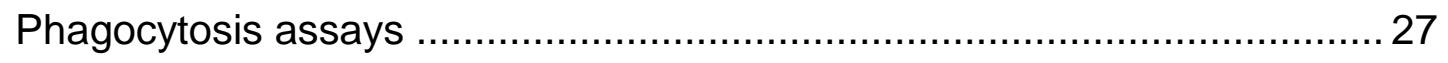

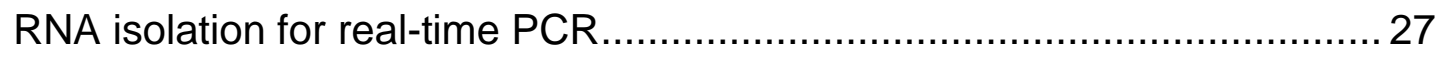

Cell isolation for flow cytometry and apoptotic cell staining.......................... 28

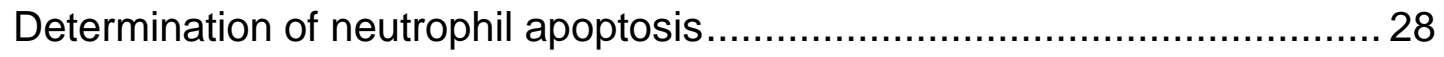

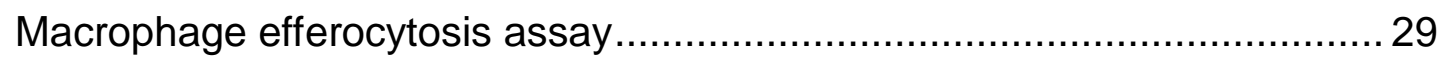

Quantification of cytokines by ELISA …………................................... 29

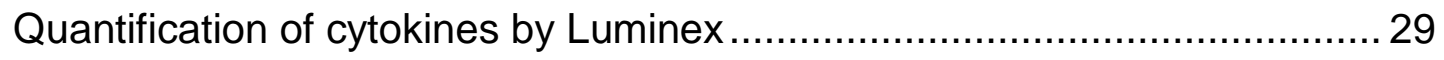

PPAR-y agonist and antagonist administration ......................................... 30

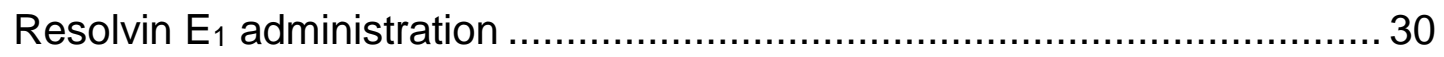

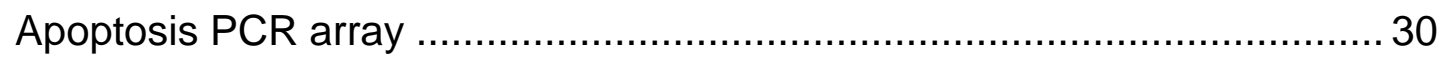

FasL and CD36 expression by flow cytometry ……..................................... 30 
CAMP ELISA

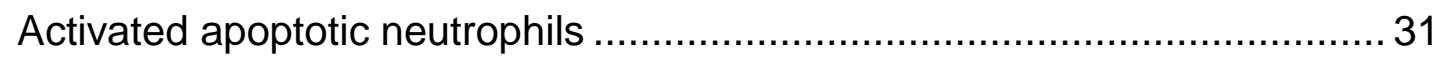

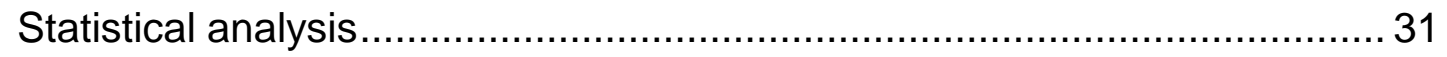

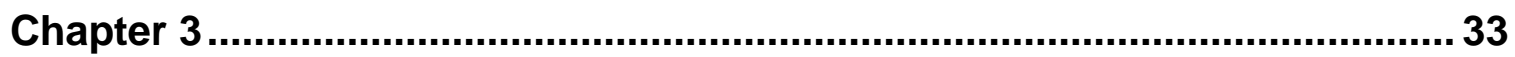

Treatment of Borrelia burgdorferi-Infected Mice with Apoptotic Cells Attenuates

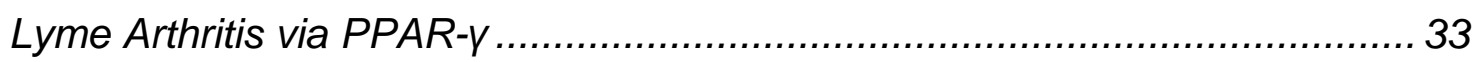

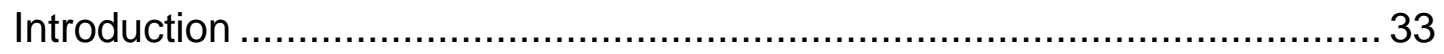

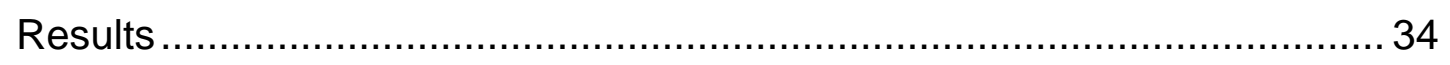

Apoptotic neutrophil and macrophage populations mimic changes in ankle

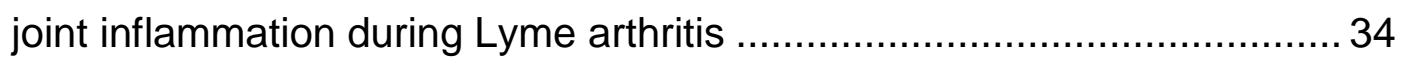

Apoptotic cell treatment limits Lyme arthritis severity and promotes

resolution but does not impact host response …................................... 36

Apoptotic cells alter macrophage and neutrophil responses in vitro .......... 39 Apoptotic cells increase PPAR-y in vitro and in vivo during B. burgdorferi infection

The PPAR-y agonist rosiglitazone alters macrophage cytokine production

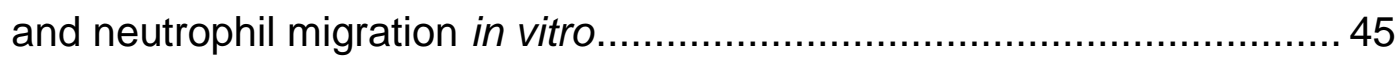

Rosiglitazone treatment reduces ankle swelling during Lyme arthritis and suppresses inflammatory cell recruitment............................................ 48

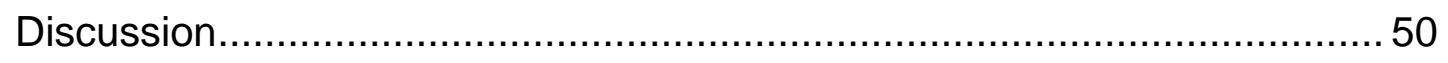

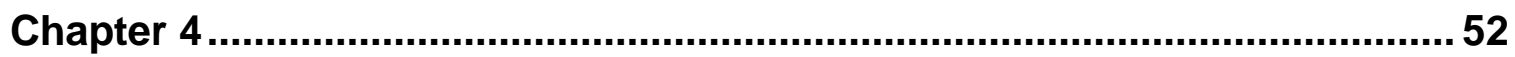


Leukotriene $B_{4}$ Signaling Through BLT1 Promotes Efferocytosis and Inflammation Resolution During Lyme Arthritis ........................................ 52

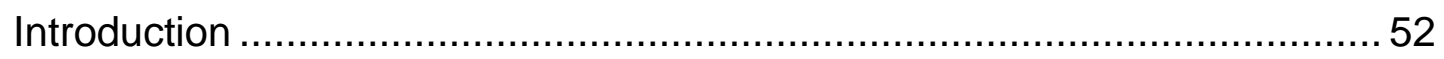

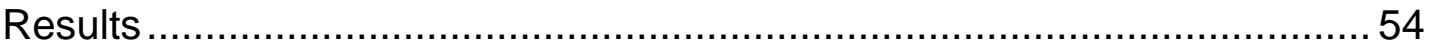

Assessment of $B$. burgdorferi infection of $\mathrm{BLT} 11^{-/-}$mice ............................ 54

Assessment of joint cellular infiltrate in $\mathrm{WT}$ and $\mathrm{BLT} 1^{-/}$mice during $B$.

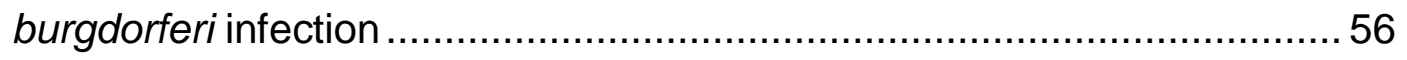

In vitro characterization of $\mathrm{BLT} 1{ }^{-/}$neutrophils .......................................... 58

Resolvin $\mathrm{E}_{1}$ restores neutrophil apoptosis and $B$. burgdorferi phagocytosis in $\mathrm{BLT1}$ - neutrophils, however it does not affect macrophage efferocytosis

BLT1 ${ }^{-/}$apoptotic BMN have decreased TNF- $\alpha$ production and DISC

formation

$\mathrm{BLT1}{ }^{-/}$apoptotic neutrophils have increased cAMP, preventing DISC

formation 67

Discussion.

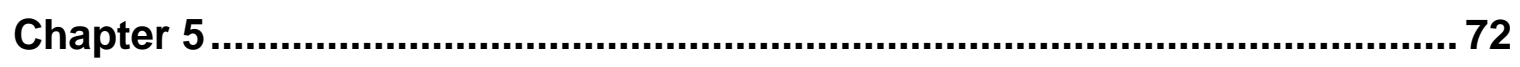

The Effects of Apoptotic Cell Secretions and Activated Apoptotic Cells on Innate Immune Cell Function .............................................................. 72

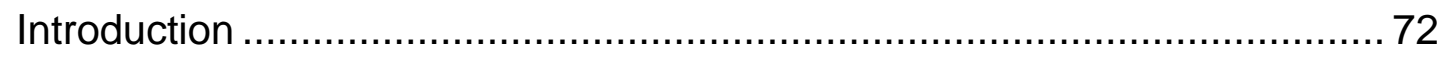

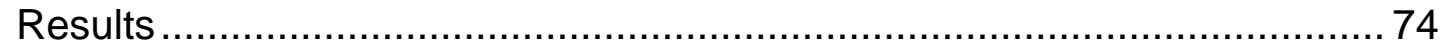

Apoptotic cell supernatant alters macrophage and neutrophil responses in vitro. 
B. burgdorferi and E. coli activated apoptotic neutrophils alter macrophage cytokine production in vitro

Activated apoptotic neutrophil supernatant alters macrophage cytokine production in vitro

B. burgdorferi-activated apoptotic neutrophils have altered cytokine production

B. burgdorferi apoptotic neutrophils are efferocytosed at the same rate as unstimulated neutrophils. 86

Discussion. 86

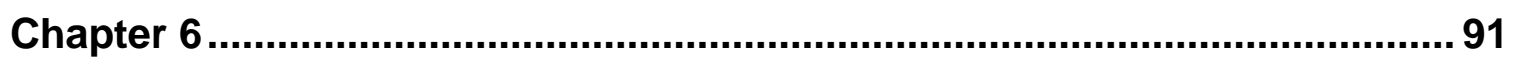

The Role of Prostaglandin $E_{2}$ during Lyme Arthritis Resolution .................... 91

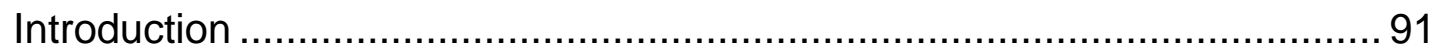

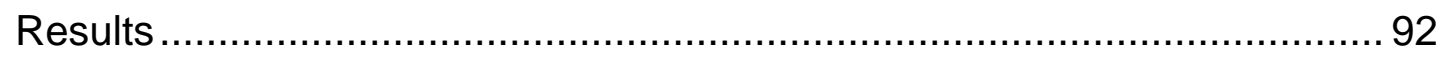
Macrophages produce $\mathrm{PGE}_{2}$ in response to $\mathrm{AC}$ and $\mathrm{AC}$ produce $\mathrm{PG} \mathrm{E}_{2}$ in vitro. 92 mPGES-1/- mice have decreased swelling and increased cellular infiltrate during inflammation resolution 94 mPGES-1/- macrophages have altered responses to apoptotic neutrophils in vitro. 99

Discussion. 104

Chapter 7 106

Discussion. 106 
Apoptotic cell detection and administration ............................................. 106

Localized administration of apoptotic cell-associated therapeutics ............ 109

Ankle swelling and cellular infiltrate are not always correlated.................. 110

The role of $\mathrm{LTB}_{4}$ signaling and $\mathrm{PGE}$ 2 production during inflammation

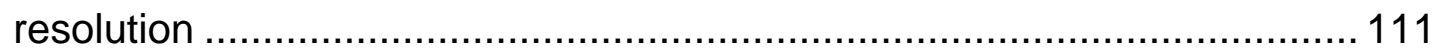

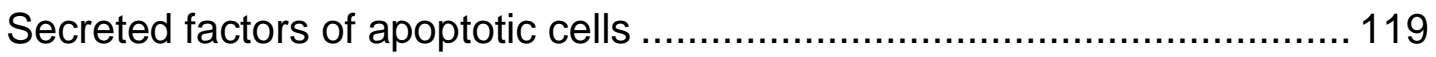

B. burgdorferi activated apoptotic neutrophils on macrophage functions in

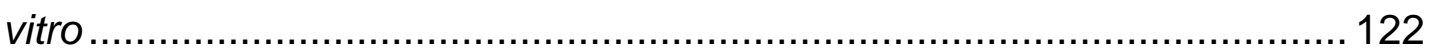

Apoptotic cells for therapeutic strategies............................................. 124

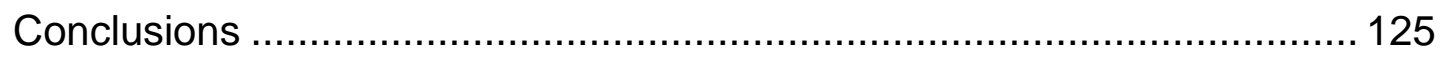

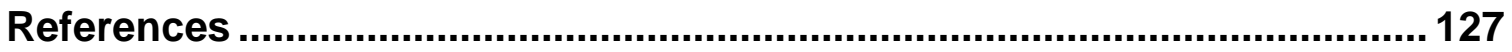

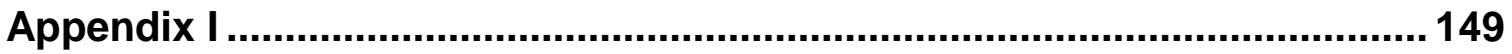

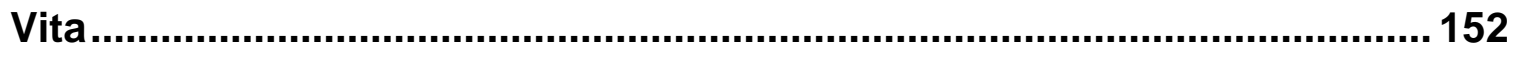




\section{List of Figures and Tables}

\section{Figures}

Figure 1: Intrinsic and extrinsic apoptosis pathways.

Figure 2: B. burgdorferi transmission cycle.

Figure 3: Quantification of apoptotic neutrophils and macrophages within $B$. burgdorferi-infected ankle joints.

Figure 4: AC treatment of $B$. burgdorferi-infected $\mathrm{C} 3 \mathrm{H}$ mice

Figure 5: In vitro quantification of cytokines in C3H BMDM supernatant cocultured with AC.

Figure 6: In vitro quantification of cytokines in supernatant of C3H BMDM co-cultured with apoptotic neutrophils.

Figure 7: In vitro AC modulation of bacterial clearance and neutrophil migration to $\mathrm{LTB}_{4}$.

Figure 8: AC induction of PPAR-y in BMDM and C3H infected mouse joints.

Figure 9: Effect of PPAR-y modulation on BMDM cytokine production. ..... 46

Figure 10: Effect of PPAR-y modulation of bacterial clearance, neutrophil migration to $\mathrm{LTB}_{4}$, and efferocytosis.

Figure 11: Rosiglitazone treatment of B. burgdorferi-infected C3H mice.... 49

Figure 12: Ankle swelling, B. burgdorferi loads and arthritis severity in $B$. burgdorferi-infected C3H WT and $\mathrm{BLT}^{-/-}$mice.

Figure 13: Quantification of neutrophils within the ankle joints of $B$. burgdorferi-infected WT and BLT1 ${ }^{-/-}$mice. 
Figure 14: Quantification of macrophages within the ankle joints of $B$. burgdorferi-infected WT and $\mathrm{BLT1} 1^{-/-}$mice.

Figure 15: Effector functions of WT and BLT1\% neutrophils and macrophages in vitro.

Figure 16: Effector functions of WT and BLT1 ${ }^{-/-}$neutrophils and macrophages in vitro with Resolvin $\mathrm{E}_{1}$

Figure 17: Cytokine production of WT and BLT1-- apoptotic neutrophils in vitro.

Figure 18: FasL and CD36 expression on WT and BLT1/-- apoptotic neutrophils in vitro and $B$. burgdorferi-infected joint neutrophils on day 14 pi in vivo.

Figure 19: Levels of cAMP in WT and BLT1 ${ }^{-/-}$apoptotic neutrophils. 69

Figure 20: In vitro quantification of cytokines in C3H BMDM supernatant cocultured with $A C$ and $A C$ supernatant.

Figure 21: In vitro $A C$ and $A C$ supernatant modulation of bacterial clearance and neutrophil migration to LTB $_{4}$

Figure 22: $A C$ and $A C$ supernatant induction of PPAR-y in BMDM. 78

Figure 23: In vitro quantification of cytokines in C3H BMDM co-cultured with apoptotic neutrophils and $B$. burgdorferi-activated apoptotic neutrophils.

Figure 24: In vitro quantification of cytokines in C3H BMDM co-cultured with apoptotic neutrophils and E.coli-activated apoptotic neutrophils.

Figure 25: In vitro quantification of cytokines in C3H BMDM co-cultured with apoptotic neutrophil supernatant and B. burgdorferi-activated apoptotic neutrophil supernatant.

Figure 26: In vitro quantification of apoptotic neutrophil and B. burgdorferiactivated apoptotic neutrophil supernatant. 
Figure 27: Effect of B. burgdorferi-activated apoptotic neutrophils on macrophage efferocytosis.

Figure 28: In vitro quantification of $\mathrm{PGE}_{2}$ in WT and Cox-2-- BMDM supernatant co-cultured with apoptotic Jurkat cells.

Figure 29: Ankle swelling in B. burgdorferi-infected WT and mPGES-1 ${ }^{-/-}$ mice.

Figure 30: Quantification of neutrophils and apoptotic neutrophils within B. burgdorferi-infected ankle joints in C3H WT and mPGES-1/- mice.

Figure 31: Quantification of macrophages and apoptotic macrophages within $B$. burgdorferi-infected ankle joints in C3H WT and mPGES-1// mice.

Figure 32: In vitro quantification of cytokines in C3H WT and mPGES-1/BMDM supernatant co-cultured with WT or MPGES-1//- apoptotic neutrophils.

Figure 33: C3H WT and mPGES-1//- BMDM efferocytosis of WT and mPGES$1^{-/-}$apoptotic neutrophils.

Figure 34: C3H WT and mPGES-1//- apoptotic neutrophil induction of PPAR$Y$ in WT and $\mathrm{mPGES}-1^{-/-}$BMDM.

Tables

Table1: PCR array of apoptosis associated gene expression in BLT1 ${ }^{-/-}$ apoptotic neutrophils compared to WT. 


\begin{abstract}
Experimental Lyme arthritis is induced by the infection of $\mathrm{C} 3 \mathrm{H}$ mice with the spirochete, Borrelia burgdorferi, and is a valuable model to study the regulation of inflammation during bacterial infection. While the lab has previously focused on how inflammation is initiated, this thesis focuses on the role of apoptotic cells during inflammation resolution in Lyme arthritis. We show administration of apoptotic cells can be used to initiate earlier resolution in $B$. burgdorferi-infected mice by decreased neutrophil recruitment, and this may be due to increased PPAR- $\gamma$ activation. Additionally, the PPAR- $\gamma$ agonist, rosiglitazone, can be used as a cell-free treatment to elicit a similar effect as the apoptotic cells. We also define the role of various eicosanoids during inflammation resolution. If apoptosis or efferocytosis, the phagocytosis of apoptotic cells, is dysregulated, it can cause exacerbated inflammation and arthritis. BLT1\% neutrophils have a defect in apoptosis and efferocytosis recognition to due decreased DISC formation. This is a result of increased cAMP in BLT1/- neutrophils due to the inability for LTB/BLT1 signaling to downregulate cAMP. Additionally, macrophages must be able to produce $\mathrm{PGE}_{2}$ to be affected by and efferocytose apoptotic cells efficiently. Deficiencies in either of these eicosanoid signaling pathways results in increased cellular infiltrate during arthritis resolution, prolonging inflammation. We also show apoptotic cells actively secrete mediators to aid in their alteration of the inflammatory response. These results demonstrate the importance of apoptosis and efferocytosis during inflammation resolution in Lyme arthritis and several mechanisms involved.
\end{abstract}




\section{Chapter 1}

\section{Introduction}

\section{Inflammation}

Inflammation is a protective reaction of the microcirculation initiated after an infection or injury [1]. Local and systemic inflammatory responses are activated to aid in the elimination of the stimulus, promote tissue repair, and establish immune memory during an infection. The acute inflammatory response involves the production and signaling of soluble mediators, including cytokines, chemokines, and eicosanoids by resident immune cells, including macrophages and fibroblasts already residing within the tissue [2, 3]. Additionally, cell adhesion molecules on circulating leukocytes are upregulated and promote an influx of granulocytes from the blood [1]. Upon arrival to the site of infection or injury, the granulocytes, primarily neutrophils, perform their primary function to phagocytose and eliminate microbes or tissue debris [1].

\section{Inflammation resolution}

Inflammation resolution is defined as the time between peak inflammatory cell influx and the clearance of these cells from the tissue site and restoration of homeostasis [4]. The recruited immune cells undergo apoptosis, while phagocytic cells, macrophages and dendritic cells, are able to phagocytose the apoptotic cells by efferocytosis, the phagocytosis of apoptotic cells [5]. This process was once thought to be passive, but increasing evidence suggest the efferocytosis of 
apoptotic cells plays a role in inflammation resolution $[3,5,6]$. Not only can efferocytosis have a negative regulation of pro-inflammatory nuclear factor kappa-light-chain-enhancer of activated B cells (NF-kB) signaling [7] and Toll-like receptor (TLR)4 expression [8], it can also increase pro-resolution signaling of peroxisome proliferator-activated receptor (PPAR)- $\mathrm{Y}[5,6]$ and prostaglandin (PG)E2 [9]. Today, many anti-inflammatory therapies are based upon inhibiting the factors responsible for driving inflammation to help combat pro-inflammatory signaling, however, this may treat the symptoms, rather than causing resolution. In addition, this limits the host's immune response, leaving treated individuals vulnerable to microbial infection. New therapeutic strategies are focusing on inhibiting pathways that breakdown pro-resolution mediators or activate proresolving mediators [1].

\section{Non-resolving inflammation}

While inflammation is important during a response to injury or a pathogen, failure to resolve inflammation can cause deleterious effects to the surrounding tissue and further immune responses. The acute inflammatory response can be divided into three phases: the productive phase, the transition phase, and the resolving phase. The productive phase involves cellular recruitment by increasing blood flow to the site of injury or infection [10]. The transition phase allows for recruited neutrophils and macrophages to perform their function, while the resolving phase returns the inflamed tissue back to homeostasis [11]. If cellular infiltration remains past their function in acute inflammation, chronic inflammation can 
occur. Chronic, or non-resolving inflammation is associated with tissue degeneration mediated by nitrogen species, lipids, cytokines, proteases, and reactive oxygen species produced by inflammatory cells [12]. No single phenomenon contributes more to the medical burden in modern society than non-resolving inflammation [13].

Non-resolving inflammation is not the primary cause of diseases such as obesity, atherosclerosis, cancer, asthma, neurodegenerative disease, multiple sclerosis or rheumatoid arthritis, but it contributes significantly to their pathogenesis [13]. Non-resolution can also occur during persistent infections. Chronic inflammation associated with tuberculosis can persist for decades, and when inflammation is extensive enough can cause severe lung damage and death [13]. The continual recruitment of immune cells, or if infiltrating immune cells fail to undergo apoptosis to limit their pro-inflammatory responses can cause further inflammation during an immune response. In addition, the inability to clear apoptotic infiltrating immune cells in a timely manner also contributes to chronic inflammation. Therefore, understanding apoptosis and the clearance of apoptotic cells is important to understand non-resolving inflammation, which contributes to a wide variety of diseases.

\section{Apoptosis and other forms of cell death}

Apoptosis, or programmed cell death, is a crucial mechanism responsible for normal cell turnover, tissue sculpting during embryogenesis, immune system 
development, and damaged cell removal $[14,15]$. Cells undergoing apoptosis will shrink without compromising the plasma membrane integrity, along with nuclear condensation and fragmentation, and bleb off into smaller pieces called apoptotic bodies [16]. Apoptotic cells and apoptotic bodies are then quickly taken up by efferocytosis, the phagocytosis of apoptotic cells, by professional phagocytes, including macrophages and dendritic cells [17]. Apoptosis is mediated by a set of caspases, which ultimately activate pro-caspase-3 into its active form, caspase3. Caspase-3 is an executioner caspase involved in apoptosis, and is responsible for chromatin condensation, nuclear fragmentation, and cytoskeletal degradation, making active caspase-3 a useful marker for the detection of apoptotic cells [18]. Regardless of how apoptosis is induced, all apoptotic cells have similar physical properties. During apoptosis, phosphatidylserine (PS), normally found on the inside of the cell membrane, is exposed on the outer cell surface. This is accomplished by a caspase-3-dependent flippase and scramblase activity which translocates phosphatidylserine to the external portion of the cellular membrane $[19,20]$.

Apoptosis differs from other forms of cell death, including necrosis, pyroptosis, and NETosis, in that they can cause an inflammatory response. During necrosis, the cells will swell, nuclear DNA will clump up or degrade, plasma membranes rupture, and cellular components are released [16]. Cells become necrotic due to changes in physiological conditions, including hypoxia, toxin exposure, exposure to reactive oxygen species, extreme temperature changes, and nutrient 
depravation [21]. Necrosis is a passive, unregulated cell death, however, necroptosis is a regulated active type of cell death [22]. Necroptosis is induced by the death receptor ligand tumor necrosis factor (TNF), and the kinases, receptor-interacting protein kinases (RIPK)1 and RIPK3, are involved in TNFinduced necroptosis [23]. RIPK1 and RIPK3 are involved in formation of the necrosome, a complex responsible for the induction of necroptosis [24]. Activation of this complex causes production of reactive oxygen species and rapid depletion of cellular adenosine triphosphate (ATP), causing mitochondrial dysfunction and subsequent cell death [25].

Pyroptosis is a caspase-dependent form of inflammatory cell death that depends on the activation of either caspase- 1 or caspase- 11 [26, 27]. Caspase- 1 is responsible for the cleavage of pro-IL-1 $\beta$ and pro-IL-18 into their active proinflammatory cytokine forms of IL-1 $\beta$ and IL-18, respectively [14]. Cells undergoing pyroptosis form a caspase-1 dependent plasma membrane pore, causing a disruption in cellular ionic gradients, increasing osmotic pressure and water influx [28]. This causes the cell to swell and eventually lyse, releasing proinflammatory cellular contents [29]. Additionally, an unidentified caspase-1activated nuclease is responsible for DNA cleavage during pyroptosis.

NETosis is a specific form of neutrophil cell death in which neutrophil extracellular traps (NETs) are released [30]. NETs are large extracellular structures composed of decondensed chromatin that provide a physical barrier to prevent 
microbial escape and recruit anti-microbial effectors [31]. Unlike apoptosis, both the nuclear and granular membranes disintegrate during NETosis, while maintaining plasma membrane integrity [32]. Also, activation of caspases does not occur during NETosis [33]. While NETs can aid in bacterial removal, they also cause a robust inflammatory response. Extracellular neutrophil elastase released by NETosis is responsible for lung tissue damage and cystic fibrosis progression [34].

\section{Forms of apoptosis}

Apoptosis can be initiated by two different pathways, intrinsic and extrinsic (Figure 1). Intrinsic apoptosis, also called mitochondrial-mediated apoptosis, is activated by a range of exogenous and endogenous stimuli, including DNA damage, ischemia, and oxidative stress. This form of apoptosis plays a role in the elimination of damaged cells [35]. The B cell lymphoma (Bcl)-2 family of proteins located on the mitochondrial membrane regulate apoptosis by the expression of pro-apoptotic mediators, like Bax and Bak, and anti-apoptotic mediators, including Bcl-2 and myeloid cell leukemia (Mcl)-1 [16]. During proapoptotic signaling, the mitochondrial membrane is perturbed, and cytochrome C is released into the cytoplasm, where it forms a complex with apoptotic protease activating factor 1 (APAF1) and pro-caspase-9 [35]. The complex cleaves procaspase- 9 into its active form, which then cleaves and activates caspase-3 [36]. 


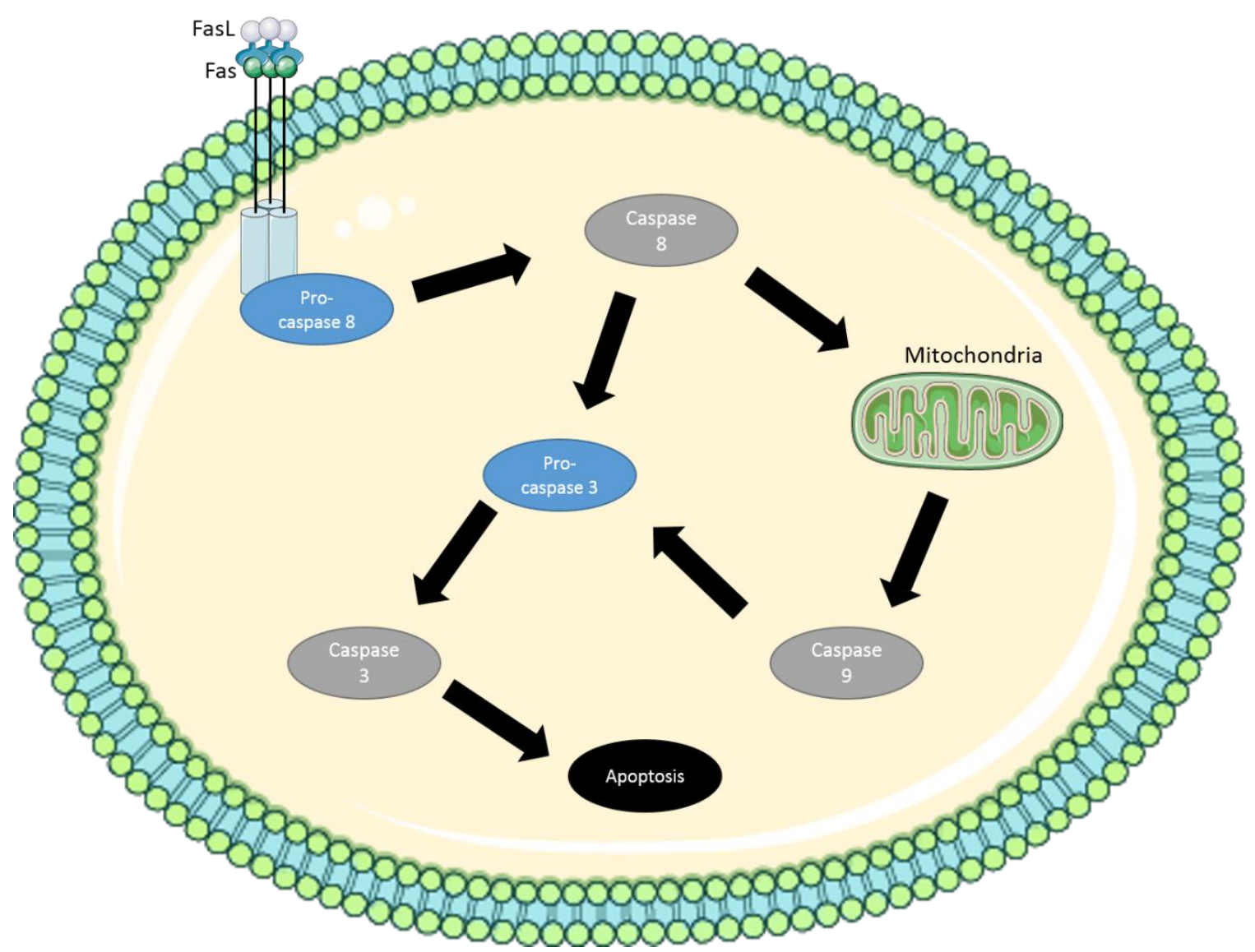

Figure 1: Intrinsic and extrinsic apoptosis pathways.

Intrinsic apoptosis is initiated through the mitochondria and activates caspase 9. Caspase 9 in turn cleaves pro-caspase 3 into its active form. The active cleavedcaspase 3 initiates apoptosis. Alternatively, extrinsic apoptosis is initiated through Fas/FasL interaction. This activates pro-caspase 8 into its active form. If there is a strong signal of caspase 8 , it will cleave pro-caspase 3 directly, or if the caspase 8 signal is weak, it will signal through the mitochondria and then follow the intrinsic apoptosis pathway. 
Extrinsic apoptosis is triggered by the TNF family of death receptors, Fas ligand (FasL), TNF- $\alpha$, and TNF-related apoptosis-inducing ligand (TRAIL) [37]. Their cytoplasmic regions contain a region called the death domain that is required for apoptosis signaling [38]. The activated death domain recruits

Fas-associated protein with death domain (FADD), pro-caspase-8, and cellular FLICE/caspase 8 inhibitory proteins (c-FLIP) [38]. The complex consisting of Fas, FADD, pro-caspase-8, and c-FLIP is called the death-inducing signaling complex (DISC) [39]. After formation of the DISC, apoptosis can be signaled in two different ways. In cells where caspase- 8 is strongly or sufficiently activated at the DISC, caspase-8 directly activates pro-caspase-3 into its active form. In cells where caspase 8 is not strongly activated, caspase- 8 will cleave Bid, another member of the Bcl-2 family, and the cleaved Bid will induce cytochrome C release from the mitochondria into the cytoplasm $[37,38]$. Cytochrome $C$ then actives the intrinsic caspase-9/caspase-3 apoptosis pathway [35].

\section{Efferocytosis}

Efferocytosis, can be broken down into four major steps [40]. First, apoptotic cells release "find me" signals to attract phagocytic cells to the location of the apoptotic cells [41]. Next, the apoptotic cell is taken up by recognition of its "eat me" signals or is not engulfed due to its expression of "do not eat me" signals [42]. During the third step the apoptotic cell is ingested by the phagocytic cell and degraded within the phagolysosome. Lastly, the post-engulfment consequences of efferocytosis, including release of anti-inflammatory cytokines and other 
mediators by the phagocyte, occur following degradation of the apoptotic cell [43].

During early apoptosis, apoptotic cells release "find me" signals, either as soluble molecules or as a part of complex extracellular vesicles [44]. "Find me" signals include: chemokines CX3CL1 and IL-8, nucleotides ATP and UTP, lipids lyso-, such as, phosphatidylcholine and sphingosine-1-phosphate (S1P), and the extracellular vesicle component, intercellular adhesion molecules (ICAM)-3 [45]. It is currently thought these "find me" signals only act over a short range, due to their short half-life due to degradation [46]. "Find me" signals are also postulated to regulate engulfment components in the recruited phagocytes and influence immunogenic vs non-immunogenic responses to apoptotic cells [47]. Cells can also release "keep out" signals, such as lactoferrin, to deter recruitment of phagocytic cells [45].

The specific recognition of dying cells among live cells depends on the identification of apoptotic cells by a class of "eat me" signals. The most well characterized "eat me" signal is PS, which trans-locates to the outside of the cell during apoptosis. PS is directly recognized by several receptors, including T cell immunoglobulin mucin-1 (TIM-1), TIM-4, and receptor for advanced glycation endproducts (RAGE) [45]. All these receptors are not ubiquitously expressed, however, therefore phagocytic cells will have variable expression of these receptors [44]. Additionally, these receptors do not bind to PS with the same 
affinity or avidity. Other "eat me" signals include annexin I and ICAM-3, which is also a "find me" signal [45]. PS can also be modified by oxidation, which allows for additional further recognition by phagocytic cells via scavenger receptors, such as CD36, CD68, and LOX-1 [48-50]. Calreticulin, which is upregulated during apoptosis, can also be recognized by the phagocytic receptor CD91 (LDLreceptor-related protein) [51]. Even in the presence of PS, there are still "don't eat me" signals that can prevent apoptotic cell recognition, including CD300a, CD31, and CD47. CD300a inhibits apoptotic cell engulfment by competitive phospholipid binding on apoptotic cells [52]. CD31 prevents ingestion of viable cells, but has an altered function when disabled [53]. CD47 inhibits apoptosis via SIRP $\alpha$, and apoptosis clearance only occurs with its disruption [51].

Following the initial contact and recognition between the phagocytic and apoptotic cell, tethering of the cells can occur to prevent detachment of the apoptotic cell. TIM-4, disabled CD31, and CD14 are all involved in the tethering process $[53,54]$. After engulfment, the phagosome becomes acidic and fuses with the phagolysosome, resulting in degradation of the apoptotic cell [55]. This stimulates the phagocytic cell to release anti-inflammatory mediators, such as IL10 , TGF- $\beta$, and $P \mathrm{PE}_{2}$, while suppressing released pro-inflammatory cytokines TNF- $\alpha$, IL-1, IL-12, and IL-8 $[6,56,57]$.

If there is a breakdown during any process of efferocytosis, the result can be chronic inflammation. If inflammatory cells, such as neutrophils, fail to undergo 
apoptosis, their inflammatory effector functions of producing pro-inflammatory cytokines and recruiting additional immune cells to the site of inflammation can be prolonged, driving further inflammation [43]. Additionally, if there is a breakdown in phagocytic cell recruitment to apoptotic cells, recognition of apoptotic cells by phagocytes, or the phagocytosis of apoptotic cells, then the apoptotic cells can undergo a secondary necrosis, furthering the inflammatory response [43]. Not only is apoptosis important for limiting inflammatory responses, but the prompt clearance of apoptotic cells contributes to the prevention of further inflammatory responses.

\section{Apoptosis during bacterial infection}

The process of apoptosis can be altered by bacteria to increase their pathogenicity. By blocking or delaying cell death, bacteria can promote their own intracellular replication and survival. For example, Legionella pneumophila replicates within alveolar macrophages, where the pathogen can deliver effectors, including Sdha and SidF, by its type IV secretion system to delay cell death. Sdha inhibits mitochondrial disruption and caspase activation of intrinsic apoptosis [58]. SidF also inhibits mitochondrial-mediated apoptosis by targeting BNIP3 and Bcl-rambo [59]. Anaplasma phagocytophilym infects neutrophils and limits apoptosis by preventing the loss of mitochondrial membrane potential, blocking the intrinsic apoptosis pathway [60]. Helicobacter pylori can block both intrinsic and extrinsic apoptosis, by upregulating pro-survival ERK signaling and Mcl-1, an anti-apoptotic factor, to aid in persistent colonization of the stomach 
mucosa [61]. Mycobacterium tuberculosis uses a two-step strategy in regulating host apoptosis. Early in infection, the pathogen post-pones cell death by upregulation of FLIP, an inhibitor of death receptor signaling, while later during infection promotes apoptosis and cell death in order to promote infection of other cells [62].

\section{Apoptosis during viral infection}

Viruses can also alter apoptosis of host cells to increase viral pathogenesis. Respiratory syncytial virus (RSV) inhibits neutrophil apoptosis by increasing expression of the anti-apoptotic Mcl-1 protein and NF-kB signaling [63]. Human cytomegalovirus (HCMV)-infected neutrophils increase their expression of extracellular adhesion molecules and ROS production to prolong inflammation, and in addition, inhibit caspase-8 by viral protein UL36 to prevent Fas-ligandmediated apoptosis [64]. During HIV/SIV infection, the frequency of neutrophil apoptosis is correlated to disease severity. Neutrophil apoptosis increases when

CD4 T cell numbers decrease, and introduction of retro-viral therapies reduces neutrophil apoptosis $[65,66]$. Influenza A virus also accelerates neutrophil apoptosis through increased expression of Fas/FasL and oxidative stressors [67]. The increase in neutrophil apoptosis may also contribute to impairment of protection against secondary bacterial infections often associated with these diseases. 


\section{Apoptosis dysregulation}

Dysregulation of apoptosis is thought to lead to several diseases with either too much or too little apoptosis, and may contribute to nearly half of all human diseases [68]. Autoimmune lymphoproliferative syndrome (ALPS) is the result of CD95 dysfunction [69]. During this disease, the immune system is unable to eliminate self-reactive lymphocytes by apoptosis and results in auto-immunity [69]. Patients with Systemic Lupus Erythematosis (SLE) have a defect in the clearance of apoptotic cells displaying autoantigens on their cell surface. This prevents the elimination of autoreactive $\mathrm{T}$ and $\mathrm{B}$ cells, resulting in tissue destruction [70]. The failed clearance of apoptotic cells also contributes to prolonged inflammatory responses during chronic obstructive pulmonary disease (COPD), pulmonary fibrosis, and cystic fibrosis [71]. Increased elastase cleavage of CD14 on the surface of macrophages contributes to reduced engulfment of macrophages in these diseases [72]. Apoptosis is induced in spinocerebellar ataxia types 1 and 3 in Huntington's disease patients, driven by increased caspase 3 expression from the Huntington protein [73]. In amyotrophic lateral sclerosis (ALS) patients, mutations causing an increase in caspase-3 expression, leads to motor neuron apoptosis [74]. Finally, most cancer cells contain changes or mutations in genes that regulate apoptosis, leading to excessive proliferation and decreased apoptosis or apoptosis resistance [75]. 


\section{Therapeutic potential of apoptotic cells}

Since apoptosis and efferocytosis play a role in the downregulation of inflammation and the anti-inflammatory nature of apoptotic cells, it has been proposed to utilize apoptotic cells as a potential therapeutic for the treatment of chronic inflammatory disease [76]. In streptococcal-cell wall induced arthritis, a model for rheumatoid arthritis, an injection of apoptotic cells was able to reduce the severity of disease by increasing levels of T regulatory cells [77]. In an inflammatory model of lung fibrosis, an instillation of apoptotic cells within the lung promoted inflammation resolution and a more positive disease outcome [5]. Additionally, therapeutics have been developed by utilizing apoptotic signaling pathways to treat diseases where apoptosis is known to be dysregulated. CD95/CD95L has been used to treat multiple sclerosis [78], and Bcl-2 proteins have been targeted for breast cancer, leukemia, and Non-Hodgkins lymphoma treatment [79]. Further characterization of the apoptotic pathway and how apoptosis and efferocytosis contributes to inflammation resolution will aid in the development of future therapeutics.

\section{Lyme Disease}

Lyme Disease is the most prevalent vector-borne disease in the United States with an estimated 300,000 new cases each year [80]. The etiological agent of Lyme Disease, Borrelia burgdorferi, is a spirochete and is transmitted to humans and animals through the bite of infected Ixodes ticks. Ixodes scapularis ticks are the primary vectors of Lyme Disease and are found in the Eastern and Central 
United States, and in addition, Ixodes pacificus ticks are located in the Western United States [81]. While the majority of Lyme Disease cases are reported from the New England and upper Midwestern states, the expansion of the geographical range of the Ixodes ticks has contributed to a 25 -fold increase in cases in humans since $1982[80,82]$. Currently, there is no vaccine available for Lyme Disease prevention in humans [83].

\section{B. burgdorferi transmission}

During the enzootic cycle of $B$. burgdorferi, Ixodes ticks undergo a three-stage life cycling, larva, nymph, and adult, to which a bloodmeal is taken at each of these stages (Figure 2) [84]. B. burgdorferi cannot be passed via transovarial transmission, from adult to egg, so each generation of tick must acquire $B$. burgdorferi after hatching [85]. Larval ticks feed on many different animals, including birds, mice, and squirrels, and B. burgdorferi is acquired through the bloodmeal from an infected animal. The tick will remain infected with $B$. burgdorferi for the remainder of its life [84]. Following the first bloodmeal, larval ticks will molt into the nymph stage and feed on its second host, which includes a similar range of animals as in the larval stage. Infected nymphal ticks can now transmit $B$. burgdorferi to new hosts, and nymphal ticks are the primary transmitter of $B$. burgdorferi to humans and companion animals, who are considered dead end or incidental hosts [85]. Shortly after the second bloodmeal, the tick will molt again into the adult stage. Adult ticks are not critical for maintaining $B$. burgdorferi in the wild due to the fact they mainly feed on larger 


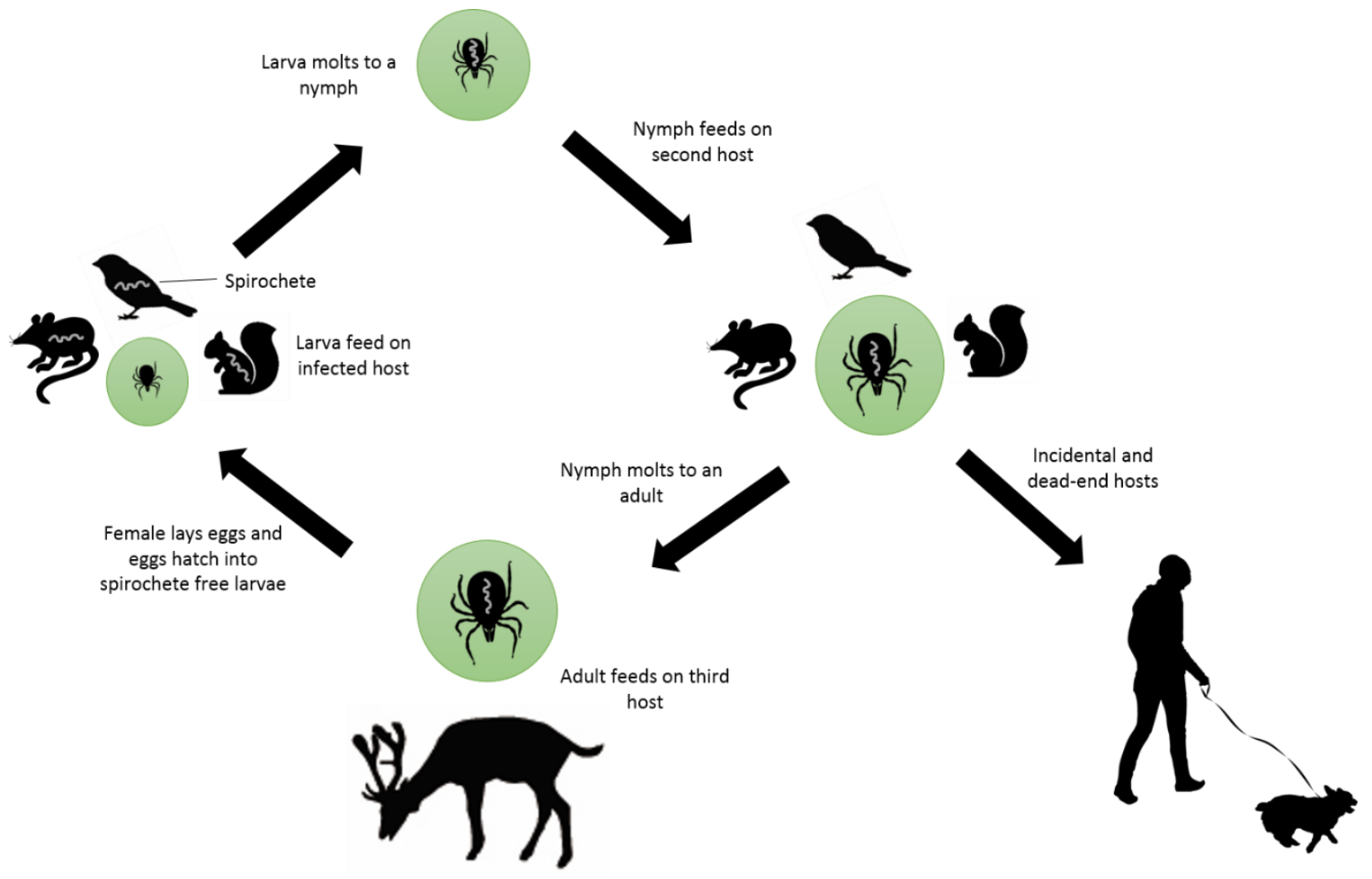

Figure 2: B. burgdorferi transmission cycle.

Adult female ticks will lay eggs, and the eggs will hatch into spirochete free larvae. Larva will feed on small animals for their first bloodmeal and will become infected if they feed on spirochete infected animals. Larva will then drop from the host and molt into nymph stage ticks. Infected nymph ticks will infect additional animals or incidental or dead-end hosts, including humans and dogs. This is the most common size of tick that transmits Lyme to humans. After their second bloodmeal, nymphs drop from their host and will molt into adult ticks. This stage of tick continues to feed on larger animals, including deer, for the remainder of their life. 
animals like deer, which are incompetent hosts for the spirochete [85]. Deer are important, however, for maintaining the tick population as, adult ticks mate on them [84].

\section{B. burgdorferi pathogenesis}

B. burgdorferi pathogenesis is determined by its expression of various outer surface proteins (Osps). While OspA is required for adherence in the tick midgut, its expression decreases upon leaving the midgut and injection into a mammalian host [86]. Meanwhile, OspC expression increases during tick transmission and is thought to play a role in the migration and survival of the spirochete [86]. Once transmitted into the host, $B$. burgdorferi replicate within the skin near the site of transmission for a short time before migrating throughout the entire body. Spirochetes will localize in various tissues throughout the body, including the joints and heart in humans [87] and the kidneys in dogs [88]. Nearly $80 \%-90 \%$ of infected humans will develop a bull's eye rash, called erythema migrans, around the site of transmission accompanied by mild flu-like symptoms [89]. Upon diagnosis, doxycycline or amoxicillin are prescribed to combat spirochete growth and to prevent more advanced symptom development [90]. Prompt treatment is critical as B. burgdorferi spread occurs rapidly within the host [87]. Around $60 \%$ of individuals which do not receive prompt antibiotic treatment, develop debilitating long-term complications, including chronic fatigue, neurological problems, carditis, and chronic arthritis $[80,91]$. B. burgdorferi has been shown to form antibiotic resistant persister cells after initial antibiotic treatment, which 
could contribute to the rise in antibiotic resistant strains found in humans and an increase in chronic Lyme symptom prevalence in treated individuals [92].

\section{Lyme arthritis}

Lyme arthritis in humans can be severe and is characterized by an increase in inflammatory cell infiltrate, usually within the weight-bearing joints of the knees and ankles, and subsequent tendonitis [93]. In the murine model of experimental Lyme arthritis, arthritis-susceptible $\mathrm{C} 3 \mathrm{H} / \mathrm{He}(\mathrm{C} 3 \mathrm{H})$ are infected with $B$. burgdorferi via the foot-pad injection in each of the hind legs, which results in swelling of the ankle joints, peaking around $3-4$ weeks post-infection [94]. C3H mice are susceptible as they develop disease, however, C57BL/6 and DBA/2 mice are resistant and display minimal inflammation [95]. Greater susceptibility to Lyme arthritis in the $\mathrm{C} 3 \mathrm{H}$ mouse strain is not due to increased infection, as spirochete clearance is similar between susceptible and resistant strains [96]. This model recapitulates a portion of the disease found in infected Lyme patients, and disease pathogenesis correlates between Lyme disease patients and this experimental mouse model [97].

Lyme arthritis development as studied in mice is mediated by innate immune cells [98], because mice without the adaptive $T$ and $B$ cells remain susceptible [99]. Development of arthritis correlates with KC/CXCR2-mediated recruitment of neutrophils into the infected joint, followed closely by inflammatory monocytes [2, 100]. A variety of pro-inflammatory mediators contribute to arthritis induction, as 
blocking these mediators resulted in decreased severity of disease [101-103]. While antibody-mediated clearance of $B$. burgdorferi from the infected joints is likely to play a role during inflammation resolution [94], the inhibition of several immune pathways resulted in poor arthritis resolution despite normal $B$. burgdorferi antibody production $[3,98,104]$. The mechanism by which inflammation resolution occurs in Lyme arthritis is currently unknown.

\section{Eicosanoids and Lyme arthritis}

Eicosanoids are locally acting bioactive signaling lipids derived from arachidonic acid (AA) and are related to polyunsaturated fatty acids (PUFA) that regulate a variety of inflammatory processes [105]. AA is released from the cellular membrane upon immune cell activation and is then metabolized into various bioactive lipids by three enzymatic pathways: lipoxygenase (LO), cyclooxygenase (COX), and cytochrome P450 (CYTP) [106]. The LO pathway is responsible for the production of pro-inflammatory leukotrienes and the proresolving lipoxins [3]. Leukotrienes are important for neutrophil recruitment and promote bronchoconstriction during airway inflammation. Lipoxins are proresolving mediators that aid in returning tissue homeostasis after an inflammatory event [4]. The COX pathway is responsible for the synthesis of the prostaglandins (PG) and thromboxanes (TX). These lipids are responsible for promoting the cardinal signs of inflammation, redness, pain, heat, and swelling, and many anti-inflammatory drugs are inhibitors of COX inhibitors, including aspirin and ibuprofen $[104,106]$. The CYTP pathway produces EETs, 
responsible for vasodilation, angiogenesis, and cellular proliferation during an infection [105].

The prostaglandin $\mathrm{PGE}_{2}$ has been previously only thought of as proinflammatory, as it seems to be an important driver of rheumatoid arthritis, however, more recent studies have determined $\mathrm{PGE}_{2}$ can either be pro- or antiinflammatory depending on which of its receptors is utilized [107]. This corresponds to upregulation of $\mathrm{PGE}_{2}$ within the joints during arthritis induction and resolution during Lyme arthritis as determined by lipidomics [108]. Additionally, both COX-2-- $[104]$ and 5 - lipoxygenase $(5-\mathrm{LO})^{-/-}$mice fail to resolve during Lyme arthritis, characterized by increased cellular infiltrate during resolution and decreased efferocytosis of apoptotic neutrophils by macrophages in 5- $\mathrm{LO}^{-/}$mice [3]. This suggests that while both pathways are responsible for pro-inflammatory mediators, they also have a secondary role during inflammation resolution. Defining the dual role of these lipid mediators during Lyme arthritis resolution will aid in a better understanding of how inflammation resolution is initiated and regulated.

\section{Purpose and experimental approach}

While much is known about how inflammation is induced in the Lyme arthritis model, very little is known how the resolution of inflammation occurs. Human Lyme arthritis patients develop a recurring, remittent arthritis with symptoms that may last for months. Lyme arthritis is an inflammatory arthritis model in which 
disease develops and then undergoes spontaneous resolution. Therefore, mechanisms of disease development and resolution can be studied in a single system using a relevant infectious organism. Understanding how inflammation resolution is initiated and the signals involved is of utmost importance for the future development of therapeutics for chronic inflammatory diseases. This research focuses on apoptotic cells and understanding their role in inflammation resolution during experimental Lyme arthritis.

The first research focus describes how administration of apoptotic cells in mice infected with B. burgdorferi promotes earlier resolution of inflammation via activation of PPAR- $\gamma$, showcasing how understanding the role of apoptotic cells can lead to potential therapeutics for inflammatory disease. The second set of data shows the importance of the pro-inflammatory eicosanoid LTB 4 and its highaffinity receptor BLT1 in signaling apoptosis and how the lack of these signals can lead to exacerbated inflammation and disease. Subsequent data showcases other important aspects of apoptotic cells, including their secretory products and their activation state prior to apoptosis. Additionally, the role of $\mathrm{PGE}_{2}$ is accessed during Lyme arthritis resolution. 


\section{Chapter 2}

\section{Materials and Methods}

\section{Mouse strains}

$\mathrm{C} 3 \mathrm{H} / \mathrm{HeJ}$ mice, 4-6 weeks of age were used as the wild type (WT) mice for all

experiments. Additionally, breeding pairs of BLT1/- (B6.129S4-Ltb4r1tm1Ald/J) (Jackson Laboratory) and mPGEs-1/- on a C57BL/6 background were backcrossed for 10 generations onto a $\mathrm{C} 3 \mathrm{H} / \mathrm{HeJ}$ background for some experiments. Both males and females were used for in vivo experiments and as a source of bone marrow cells. The mice were bred and housed in a specific pathogen-free facility and given continuous access to food and water. At specific time points following infection, mice were humanely euthanized via $\mathrm{CO}_{2}$ inhalation as accepted by American Veterinary Medical Association guidelines. All studies were conducted in accordance with the guidelines of ACUC of the University of Missouri and performed under an approved protocol.

\section{Bacteria and infections}

Spirochetes from the N40 strain of $B$. burgdorferi were used for all infections [109]. Frozen stocks stored at $-80^{\circ} \mathrm{C}$ were added to $7 \mathrm{ml}$ of complete BarbourStoenner-Kelly-H medium containing 6\% rabbit serum (Sigma) and grown for 6 days to log phase at $32^{\circ} \mathrm{C}$. Spirochetes were enumerated using dark-field microscopy and a Petroff-Hausser counting chamber (Hausser Scientific, Horsham, PA). For all in vivo infections, dilutions were made in sterile Barbour- 
Stoenner-Kelly-H medium so that each hind footpad was inoculated with $50 \mu \mathrm{l}$ of medium containing $5 \times 10^{4}$ B. burgdorferi. GFP-expressing B. burgdorferi (a kind gift from J. Carroll [110], NIH), was used for all phagocytosis assays. $B$. burgdorferi was given at a multiplicity of infection (MOI) of 10 for all in vitro studies.

\section{Assessment of arthritis pathology}

Progression of the arthritis was monitored by measuring the ankle swelling of the thickest cranio-caudal portion of the joint by use of a metric caliper. The increase in diameter of the ankle was determined by measuring the joint one day prior to infection and subtracting this value from the measurement post-infection. Ankles were harvested on indicated days and fixed in $10 \%$ zinc-formalin, paraffin embedded and stained with hematoxylin and eosin. Sections were evaluated in a blinded manner for disease severity on scale of 0 to 4 , with 0 representing no inflammation and 4 representing severe inflammation, as previously described [2].

\section{Determination of tissue B. burgdorferi loads}

Ankles were harvested and snap-frozen in liquid nitrogen and homogenized in TRIzol reagent according to the manufacturer's protocol. Multiplex real-time PCR was performed using SYBR Green PCR Master Mix (Applied Biosystems) according to the manufacturer's instructions. Reactions for $B$. burgdorferi flagellin were normalized to copies of nidogen DNA within the same sample, as 
previously described [100]. Bacterial loads are expressed as copies of $B$. burgdorferi flagellin per 1000 copies of mouse nidogen [2].

\section{Isolation of bone marrow-derived macrophages}

Bone marrow was isolated from mouse tibias and femurs by flushing the bones with sterile Hank's Balanced Salt Solution (HBSS) (Life Technologies) containing $2 \%$ fetal bovine serum (FBS) (Sigma). Isolated bone marrow was cultured for 6 days in RPMI 1640 supplemented with 30\% L929 cell-conditioned medium, 10\% FBS, and $2 \%$ penicillin-streptomycin (Life Technologies) at $37^{\circ} \mathrm{C}$ in $5 \% \mathrm{CO}_{2}$. Adherent cells were scraped, washed, plated, and left overnight at $37^{\circ} \mathrm{C}$ in $5 \%$ $\mathrm{CO}_{2}$ in RPMI 1640 supplemented with 10\% FBS and 2\% penicillin-streptomycin.

\section{Neutrophil extraction}

Neutrophils were isolated as previously described [2]. Briefly, bone marrow was harvested as stated above from mouse tibias and femurs and separated on a 1-

$\mathrm{ml}$ Histopaqe-1119 and 5-ml Histopaque-1038 (Sigma) gradient at $700 \times \mathrm{g}$ for 30 minutes at room temperature (RT). ACK lysis buffer was added to the last $1.5 \mathrm{ml}$ of the gradient, incubated for 3 minutes at RT, and spun down at $500 \times \mathrm{g}$ for 5 minutes. For CFSE-labeled neutrophils, the CellTrace Far Red Cell Proliferation Kit (Thermo Fisher) was used according to the manufacturer's instructions. 


\section{Apoptotic cells}

For indicated experiments, Jurkat cells were grown in complete media containing RPMI $1640,10 \% \mathrm{FBS}$, and $2 \%$ penicillin-streptomycin at $37^{\circ} \mathrm{C}$ in $5 \% \mathrm{CO}_{2}$ until needed. Jurkat cells were pelleted at $300 \times \mathrm{g}$ at $4^{\circ} \mathrm{C}$ for 5 minutes, and the supernatant was discarded. Cells were resuspended in complete media and washed three times. Cells were resuspended in $1 \mathrm{ml}$ of RPMI 1640 containing $10 \%$ FBS and CD95 Fas Ab (Biolegend) at $10 \mu \mathrm{l} / \mathrm{ml}$. After 6 hours, cells were counted and diluted to a concentration of $1 \times 10^{6}$ apoptotic cells (AC)/ml. Ten microliters was injected into the tibio-tarsal joint synovial cavities of each mouse on indicated days for in vivo experiments. For apoptotic neutrophils, bone marrow neutrophils (BMN) were harvested as described above and left for 24 hours in complete media containing RPMI 1640, 10\% FBS, and $2 \%$ penicillinstreptomycin at $37^{\circ} \mathrm{C}$ in $5 \% \mathrm{CO}_{2}$. AC were verified via flow cytometry by cleavedcaspase $3^{+}$cells. AC were used at a 2:1 (AC:cells) concentration for the in vitro studies.

\section{Protein extraction}

Protein from joints was harvested as previously described [100], and tissues were harvested and snap-frozen in liquid nitrogen. The frozen samples were wrapped in aluminum foil and pulverized with a hammer, then placed into $1 \mathrm{ml}$ of cold homogenization buffer, HBSS containing $0.2 \%$ protease inhibitor cocktail (Sigma). Samples were homogenized for 2 minutes on a bead beater and then sonicated on ice for 20 seconds at $60 \%$ amplitude. Centrifugation $(2000 \mathrm{xg}$ for 
20 minutes, $4^{\circ} \mathrm{C}$ ) and filtration through a $0.45-\mu \mathrm{m}$ filter were then performed.

Protein quantity was then assessed via a BCA protein quantification assay (Thermo Scientific). Samples were stored at $-20^{\circ} \mathrm{C}$ until used for analysis.

\section{Western blot}

Total protein was isolated as described above, and sample proteins $(25 \mu \mathrm{g})$ were separated on a $10 \%$ SDS-PAGE gel and transferred electrophoretically to a Trans-Blot nitrocellulose membrane (Bio-Rad). Membranes were blocked with 5\% milk in TBST, then incubated with a 1:1000 dilution of anti-mouse PPAR- $\nu$ E8 (Santa Cruz Biotechnology) or 1:1000 $\beta$-actin C4 (Santa Cruz Biotechnology) antibody (Ab). Secondary Ab was used as a 1:2000 dilution of goat $\alpha$-mouse (Santa Cruz Biotechnology). The blot was developed using a Pierce ECL Western Blotting Substrate (Thermo Scientific) and imaged via x-ray film. Densitometry on bands was performed using ImageJ software.

\section{Neutrophil migration assay}

Neutrophil migration assays were performed as previously described [111]. BMN were harvested as described above and were incubated with $A C$ at a 2:1 concentration for 2 hours. Fifty nanograms per milliliter of leukotriene $\mathrm{B}_{4}\left(\mathrm{LTB}_{4}\right.$; Cayman Chemical) was added to the bottom of a 24-well Trans-well plate with a 3.0- $\mu$ m polyester membrane (Greiner). Neutrophils were added to the top of the Trans-well and allowed to migrate for 2 hours. Cells were then collected from the bottom of the well, and the number of neutrophils migrating through the 
membrane was determined by flow cytometry staining for CD45.2 (eBioscience) and Ly6G (BD Biosciences).

\section{Phagocytosis assays}

Neutrophil and macrophage phagocytosis of $B$. burgdorferi was determined by isolating bone marrow-derived macrophages (BMDM) and BMN as previously described and preincubating with $\mathrm{AC}$ at a 2:1 concentration for 1 hour prior to the addition of GFP-expressing B. burgdorferi for the indicated times at a MOI of 10 . Cells were washed and stained for CD45.2 (eBioscience), F4/80 (eBioscience) for macrophages, and Ly6G (BD Biosciences) for neutrophils for flow cytometry analysis. The percentage of cells phagocytosing $B$. burgdorferi was determined by the number of $\mathrm{GFP}^{+}$cells over the total number of cells, expressed as a percentage.

\section{RNA isolation for real-time PCR}

RNA was isolated from cells using the RNeasy Mini Kit (Qiagen) according to the manufacturer's protocol. RNA was synthesized into cDNA using the SuperScript III First-Strand Synthesis system for RT-PCR (Invitrogen) according to the manufacturer's instructions. Sample cDNA was then diluted to $50 \mathrm{ng} / \mu \mathrm{l}$ and realtime PCR performed using SYBR Green (Life Technologies). PPAR $-\mu$ and CD36 cycle threshold (CT) values were normalized to GAPDH CT values, with $\triangle \triangle C T$ values shown as fold change compared with unstimulated cells. 


\section{Cell isolation for flow cytometry and apoptotic cell staining}

Inflammatory cells were isolated from ankle joints as previously described [112]. Ankles were removed and placed in $15-\mathrm{ml}$ conical tubes containing $5 \mathrm{ml}$ of $1 \mathrm{X}$ PBS with $4 \% \mathrm{FBS}, 0.03 \mathrm{mg}$ of DNase I (Sigma), and $30 \mu \mathrm{l}$ of $100 \mathrm{mg} / \mathrm{ml}$ stock collagenase/dispase (Roche). Samples were placed on a rocker for 1 hour at RT. Samples were then placed into sterile petri dishes with RPMI 1640 containing $10 \%$ FBS and flayed apart using sterile forceps. Cells were strained through a 70- $\mu \mathrm{m}$ filter (Greiner) and washed three times. Live cells were determined by counting using trypan blue exclusion. A total of $1 \times 10^{6}$ cells were stained in a 96well U-bottom plate (Greiner), with all samples treated with Fc Block (antiCD16/CD32; eBioscience), and stained for CD45.2 PE (eBiosciences), F4/80 APC (eBioscience) and Ly6G PE-Cy7 (BD Biosciences). Cells were washed and fixed in $4 \%$ paraformaldehyde for 15 minutes. For intra-cellular stains, cells were permeabilized with saponin prior to staining with cleaved caspase-3 Alexa Fluor 488 (Cell Signaling Technology). Joint cellular infiltrate was analyzed using a Dako CyAn Flow Cytometer and Summit V5.0 software.

\section{Determination of neutrophil apoptosis}

$\mathrm{BMN}$ were harvested as described above and left for indicated times at $37^{\circ} \mathrm{C}$ in $5 \% \mathrm{CO}_{2}$. Apoptotic neutrophils were determined via flow cytometry by cleaved caspase- $3^{+}$neutrophils. 


\section{Macrophage efferocytosis assay}

BMN were harvested as described and labeled with CellTrace Far Red Cell Proliferation Kit (APC) (Thermo Fisher), used according to the manufacturer's instructions, and left for 24 hours at $37^{\circ} \mathrm{C}$ in $5 \% \mathrm{CO}_{2}$. Apoptotic neutrophils were verified via flow cytometry by cleaved caspase $-3^{+}$cells. Apoptotic BMN were

given to BMDM at a 2:1 concentration and spun at $300 \times g$ for 5 minutes at $4^{\circ} \mathrm{C}$. After 4 hours, cells were washed and stained for CD45.2 PE (eBioscience) and F4/80 APC-Cy7 (eBioscience) for flow cytometry analysis. The percentage of macrophages efferocytosing an apoptotic neutrophil was determined by the number of $\mathrm{APC}^{+}$macrophages over the total number of macrophages, expressed as a percentage.

\section{Quantification of cytokines by ELISA}

Cytokine levels were measured from cell supernatants or ankle joint homogenates [100] using TNF- $\alpha$ (applied Biosystems), IL-10 (BD Biosciences), and KC (R\&D Biosystems) ELISA kits. Results were reported in picograms per milliliter or picograms per milligram protein.

\section{Quantification of cytokines by Luminex}

A Luminex assay (R\&D Biosystems) was used to measure apoptotic neutrophil production of KC, TNF- $\alpha$, and IL-10 per manufacturer's instructions. 


\section{PPAR- $\gamma$ agonist and antagonist administration}

The PPAR- $\gamma$ agonist, rosiglitazone, and antagonist, GW 9662 (Cayman

Chemical) were used at a $10 \mu \mathrm{M}$ concentration for all in vitro experiments. For in vivo experiments, stock rosiglitazone was diluted in sterile PBS to $1 \mathrm{mg} / \mathrm{ml}$, and $10 \mu \mathrm{l}$ was injected into the tibio-tarsal joint synovial cavities of each mouse on 11 and 18 days post-infection.

\section{Resolvin $\mathrm{E}_{1}$ administration}

Resolvin $\mathrm{E}_{1}$ (Cayman Chemical) was used at 10nM for all in vitro experiments and given 1 hour prior to the addition of GFP $B$. burgdorferi during the neutrophil phagocytosis assay. During macrophage efferocytosis, apoptotic neutrophils were cultured in 10nM Resolvin $E_{1}$, and BMDM were given 10nM Resolvin $E_{1}, 3$ hours prior to the addition of the apoptotic neutrophils.

\section{Apoptosis PCR array}

RNA was isolated from cells and synthesized into cDNA as stated above. cDNA was used in the $\mathrm{RT}^{2}$ Profiler PCR Array for Apoptosis genes (Qiagen) according to the manufacturer's instructions. Gene expression values are expressed as fold change compared to WT apoptotic neutrophils.

\section{FasL and CD36 expression by flow cytometry}

In vitro, $\mathrm{BMN}$ were harvested as described above, and left for 24 hours at $37^{\circ} \mathrm{C}$ in $5 \% \mathrm{CO}_{2}$. In vivo, mice were infected as stated above, and joints were harvested 
and processed as described above on indicated days post-infection. Cells were stained in a 96-well U bottom plate (Greiner) with all samples treated with Fc Block (anti-CD16/CD32) (eBioscience) for 15 minutes at $4^{\circ} \mathrm{C}$. Cells were then stained with the following antibodies: Ly6G PE-Cy7 (BD Biosciences), CD178/FasL PE (Biolegend) and CD36 Alexa 488 (Biolegend). MFI of both FasL and CD36 was measured on CD45.2+, Ly6G+ cells.

\section{CAMP ELISA}

WT and BLT1 ${ }^{-}$BMN were harvested as described and left for 24 hours at $37^{\circ} \mathrm{C}$ in $5 \% \mathrm{CO}_{2}$. cAMP Complete ELISA kit (Enzo) was used to measure intracellular cAMP according to the manufacturer's instructions.

\section{Activated apoptotic neutrophils}

Neutrophils were harvested as stated above and given B. burgdorferi or Escherichia coli at a $\mathrm{MOI}$ of 10 . The cells were left for 24 hours at $37^{\circ} \mathrm{C}$ in $5 \%$ $\mathrm{CO}_{2}$ in a 96-well U bottom plate (Greiner). Activated apoptotic neutrophils were given to BMDM at a 2:1 concentration along with B. burgdorferi at a MOI of 10 .

\section{Statistical analysis}

In vivo experiments have groups of $n=4$ and were performed in duplicate or triplicate, and in vitro experiments were performed in duplicate or triplicate with $n=3$. Statistical significance for comparing multiple groups with a single control was assessed by ANOVA followed by a Dunnett test, whereas significance 
among multiple groups was assessed by ANOVA followed by a Bonferroni posttest. Additional data consisting of two groups used an unpaired, two-tailed, Student $t$ test. Statistical significance of differences in histology scores was determined by a Mann-Whitney $U$ test. Significance levels are set at $p<0.05$. 


\section{Chapter 3}

\section{Treatment of Borrelia burgdorferi-Infected Mice with Apoptotic Cells Attenuates Lyme Arthritis via PPAR- $\gamma$}

\section{Introduction}

Murine Lyme arthritis develops as the result of the infiltration of innate immune cells, primarily neutrophils and macrophages. These phagocytic cells are efficient in their uptake and killing of $B$. burgdorferi both in vitro and in vivo $[113,114]$. Development of arthritis correlates with KC/CXCR2-mediated recruitment of neutrophils to the infection joint, followed by inflammatory monocytes [100]. While much is known about arthritis induction, little is known about how inflammation resolution occurs. Neutrophils are short-lived inflammatory cells programmed to undergo apoptosis to limit their pro-inflammatory response. The clearance of apoptotic neutrophils by macrophages, efferocytosis, switches them from a pro- to an anti-inflammatory state. Additionally, apoptotic cells (AC) have been shown to exhibit anti-inflammatory properties and aid in the induction of resolution in other models of inflammation $[5,77]$. Quantification of AC in the joints of $B$. burgdorferi-infected mice has not been reported, however, the clearance of these cells is likely to play an important role in mediating arthritis resolution and aid in returning the tissue to homeostasis.

The mechanism by which AC elicit an anti-inflammatory effect is still being determined, however, PPAR-y may be involved. PPAR-y is a nuclear receptor 
that mediates transcriptional activation and suppression and is expressed in a variety of immune cells, including neutrophils and macrophages [115]. It has been shown to suppress inflammatory gene expression in macrophages and chemotaxis in neutrophils among other factors $[116,117]$. PPAR-y activation has been shown to increase after $A C$ administration in a lung fibrosis model, contributing to the down-regulation of pro-inflammatory cytokines, promoting inflammation resolution and decreased disease severity [5]. Thus, this study will focus on the impact of exogenous administration of AC to promote earlier inflammation resolution in $B$. burgdorferi-infected mice and examine the role of AC-mediated PPAR- $\gamma$ activation during Lyme arthritis resolution.

\section{Results}

\section{Apoptotic neutrophil and macrophage populations mimic changes in ankle joint inflammation during Lyme arthritis}

To determine if the number of $A C$ within the infected joint changes over the course of infection, ankle joints of $\mathrm{C} 3 \mathrm{H}$ B. burgdorferi-infected mice were processed for flow cytometry and stained for apoptotic neutrophils and macrophages. The number and percentage of apoptotic neutrophils within the infected ankle joints significantly increased over the course of infection (Figure $3 \mathrm{~A}, 3 \mathrm{C}$ ), as did the number and percentage of apoptotic macrophages (Figure 3B, 3D). Both apoptotic neutrophils and macrophages increased significantly around day 21 post-infection (pi) and peaked at day 28 . This correlated well with the peak of inflammation, which usually occurs around day 21 in this model. By 

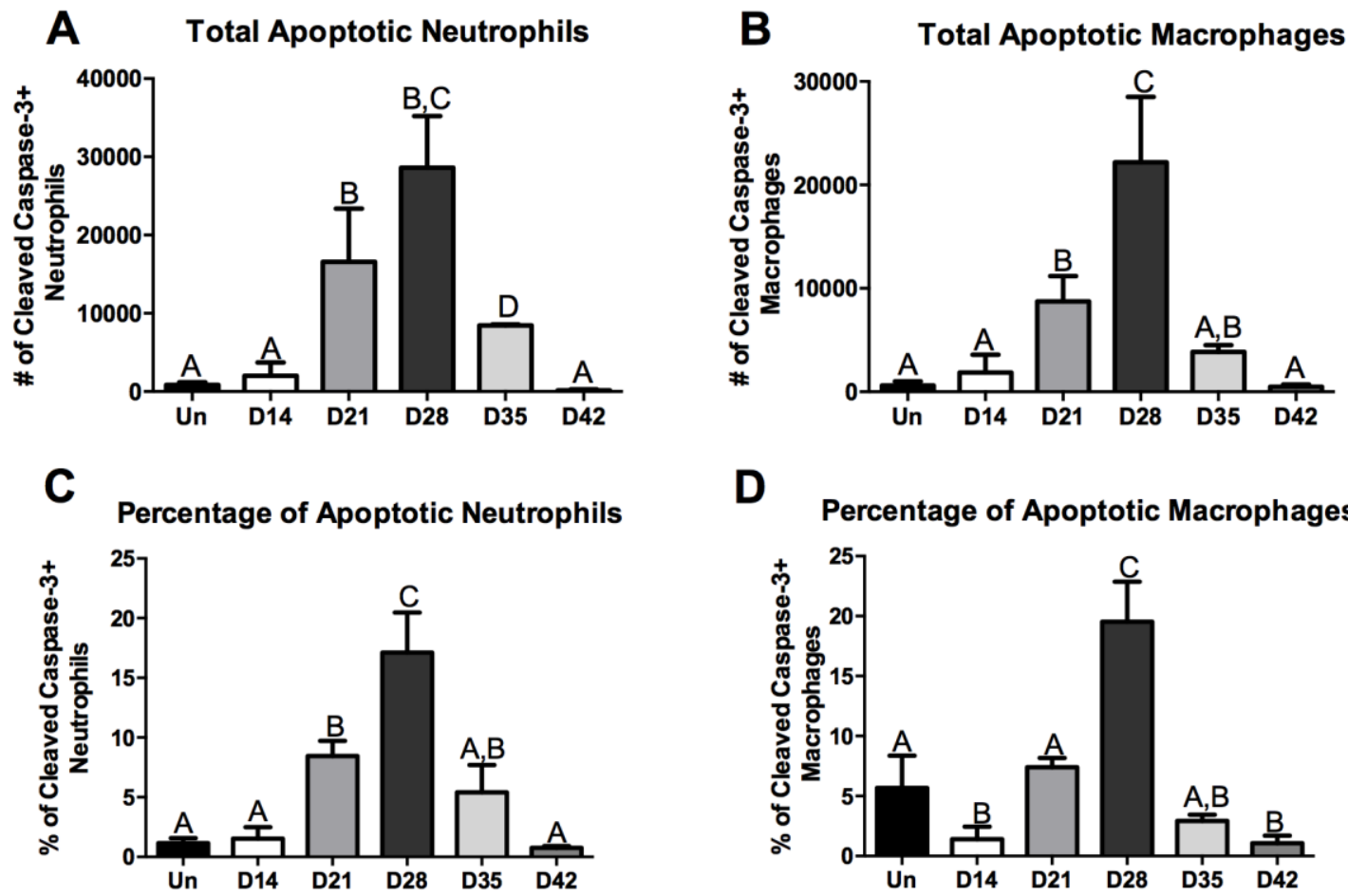

Figure 3: Quantification of apoptotic neutrophils and macrophages within B. burgdorferi-infected ankle joints.

$\mathrm{C} 3 \mathrm{H}$ mice were infected with $1 \times 10^{5} \mathrm{~B}$. burgdorferi and sacrificed at indicated days for flow cytometry analysis of joint inflammatory cells. Total cleavedcaspase $-3^{+}$neutrophils (A) and macrophages (B) and percentages of cleavedcaspase $-3^{+}$neutrophils (C) and macrophages (D) of total CD45.2+ inflammatory cells were evaluated on $14,21,28,35$, and 42 days pi. $n=4$ per group. Bars with different letters are significantly different from others at the $p<0.05$ level. 
day 35 , most of the AC had been cleared, which correlates well with arthritis resolution.

\section{Apoptotic cell treatment limits Lyme arthritis severity and promotes resolution but does not impact host response}

Ankle swelling is a convenient way to monitor inflammatory changes within the $B$. burgdorferi-infected joint [99]. To determine if AC administration would alter Lyme arthritis progression, AC, (apoptotic Jurkat cells), were administered via intraarticular ankle injection on 11 and 18 days pi. These timepoints were chosen to avoid downregulation of neutrophil and macrophage effector functions during times of $B$. burgdorferi clearance within the ankle joints [3] and to be given earlier than when the increase of apoptotic neutrophils and macrophages occurs during resolution (Figure 3). Ankle swelling, B. burgdorferi loads within the ankle, and inflammatory infiltrates and cytokines within the ankle joints were assessed at various time points throughout the infection. As shown in Figure 4, AC or live Jurkat cell administration had little or no effect on ankle swelling in uninfected mice (Figure 4A), whereas the administration of $A C$ to $B$. burgdorferi-infected mice significantly reduced ankle swelling at 21,28 , and 35 days pi compared with infected controls (Figure 4B). The addition of live Jurkat cells had no impact on ankle swelling in infected mice throughout the infection (Figure 4B). Similarly, the addition of exogenous $A C$ did not impact the host immune response to $B$. burgdorferi infection, as clearance of $B$. burgdorferi from tissue within the ankle joints throughout infection was not altered (Figure 4C). However, analysis of the 
A

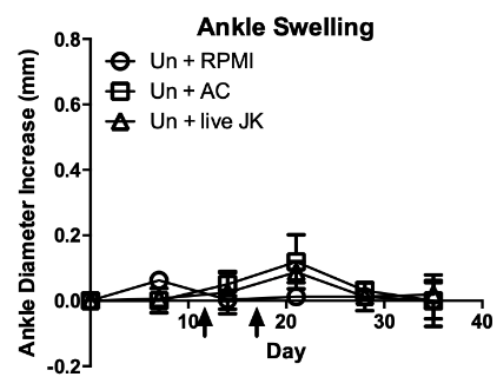

B

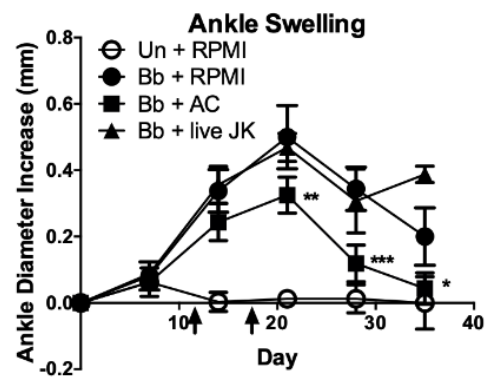

C

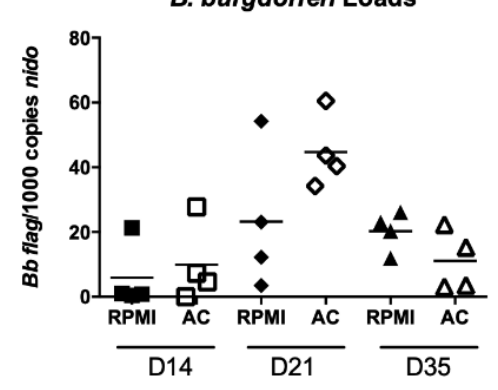

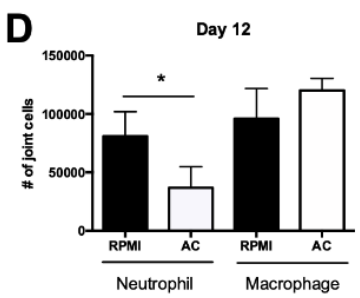
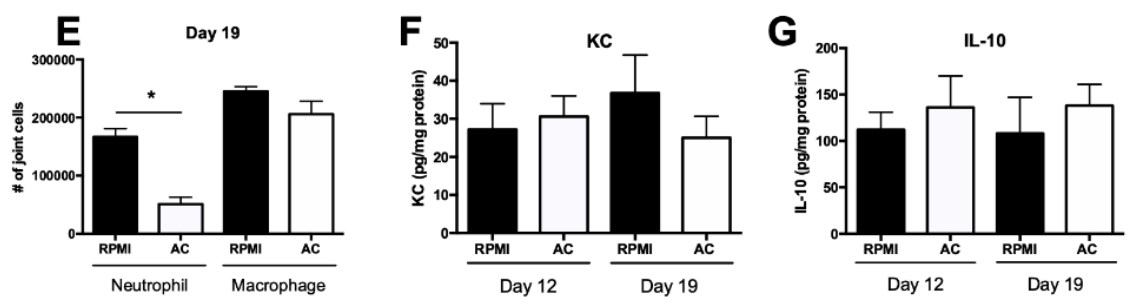

Figure 4: AC treatment of $B$. burgdorferi-infected $C 3 \mathrm{H}$ mice.

$\mathrm{C} 3 \mathrm{H}$ mice were uninfected or infected with $1 \times 10^{5} \mathrm{~B}$. burgdorferi and given apoptotic Jurkat cells (AC), live Jurkat cells (live JK) or RPMI 1640 control on days 11 and 18 pi. Ankle swelling was monitored throughout the experimental time course in uninfected (A) or infected (B) mice. Quantification of $B$. burgdorferi loads in the ankle joints was assessed at indicated days pi (C). Infiltrating neutrophils and macrophages were quantified on 12 (D) and 19 (E) days pi by flow cytometry. Levels of $K C(F)$ and IL-10 $(G)$ were quantified from joint tissue on days 12 and 19 pi. $n=4$ per group. Asterisk (*) symbols indicate differences from infected controls animals at the same time point. ${ }^{*} p<0.05,{ }^{* *} p<0.01$, and ${ }^{\star * *} p<0.001$. 
joint inflammatory infiltrate on days 12 and 19 pi showed a significant decrease in neutrophils within the joints of AC-treated mice compared with control-treated animals, whereas macrophage number remained unchanged (Figure 4D, 4E), suggesting the presence of the increased number of $A C$ within the joint can limit inflammatory processes by decreased neutrophil recruitment and promote earlier resolution.

To gain more insight into the mechanism, we attempted to measure cytokine production within the infected joint [100]. While the joints expressed no detectable levels of TNF- $\alpha$, similar to a previous report from our lab [100], even though TNF- $\alpha$ is typically produced by activated macrophages and is reduced in the presence of $A C$ [118]. We have similarly shown that the production of $K C$ is critical for neutrophil recruitment and the development of Lyme arthritis [2, 119]. However, we did not find an effect of $\mathrm{AC}$ treatment on $\mathrm{KC}$ levels in the joint (Figure 4F). Efferocytosis is known to increase macrophage production of IL-10 and promote inflammation resolution [120]. We found a trend toward higher IL-10 levels in the joints of AC-treated mice, but these were not statistically significant (Figure 4G). Altogether, these data show that treatment of B. burgdorferi-infected mice with $\mathrm{AC}$ attenuated Lyme arthritis development and promoted disease resolution without a detrimental effect on host response. 


\section{Apoptotic cells alter macrophage and neutrophil responses in vitro}

We next wanted to determine the effect of $A C$ clearance on unactivated bone marrow-derived macrophages (BMDM) or those activated by co-culture with live B. burgdorferi. Unactivated BMDM made no detectable levels of TNF- $\alpha$, or KC and only low levels of IL-10, and these were not influenced by the addition of AC to the culture (Figure 5A-C). BMDM activated by co-culture with $B$. burgdorferi, however, made high levels of TNF- $\alpha$ and KC and moderate levels of IL-10. The addition of $A C$ to these cultures significantly decreased TNF- $\alpha$ and $K C$ levels and increased IL-10 levels (Figure 5A-C). Nearly identical results were seen when apoptotic neutrophils were used rather than apoptotic Jurkat cells (Figure 6A-C), similar to a previous report using bleomycin-activated cells [5]. Therefore, even when $B$. burgdorferi are present, the uptake of $A C$ can significantly decrease macrophage inflammatory responses.

We next wanted to determine how AC impacted macrophage and neutrophil function, including phagocytosis of $B$. burgdorferi and chemotaxis. As shown in Figure $7 \mathrm{~A}$, the presence of $\mathrm{AC}$ decreased the percentage of macrophages phagocytosing B. burgdorferi. This was true for neutrophils as well (Figure 7B), with a significant decrease in the percentage of neutrophils phagocytosing $B$. burgdorferi compared with controls. Live Jurkat cells had no effect on macrophage or neutrophil phagocytosis of B. burgdorferi (Figure 7A, 7B). Additionally, we wanted to see if AC would impact neutrophil migration to the neutrophil chemoattractant Leukotriene $\mathrm{B}_{4}\left(\mathrm{LTB}_{4}\right)$, known to play an important 

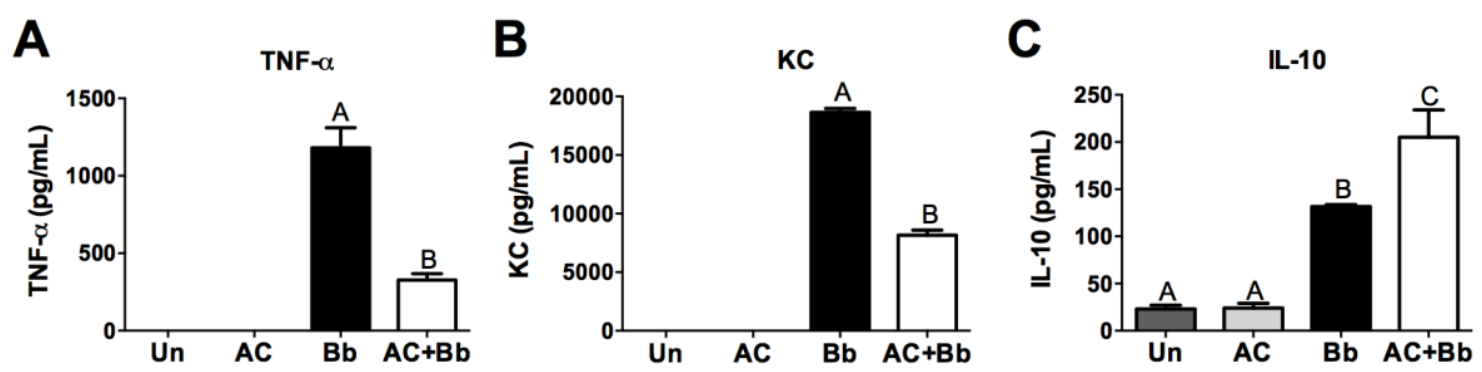

Figure 5: In vitro quantification of cytokines in C3H BMDM supernatant cocultured with AC.

C3H BMDM were co-cultured with apoptotic Jurkat cells and B. burgdorferi for 24 hours, and cytokine levels of TNF- $\alpha(A), K C(B)$, and IL-10 (C) were quantified by ELISA. $n=3$ per group. Bars with different letters are significantly different from each other at the $p<0.05$ level. 

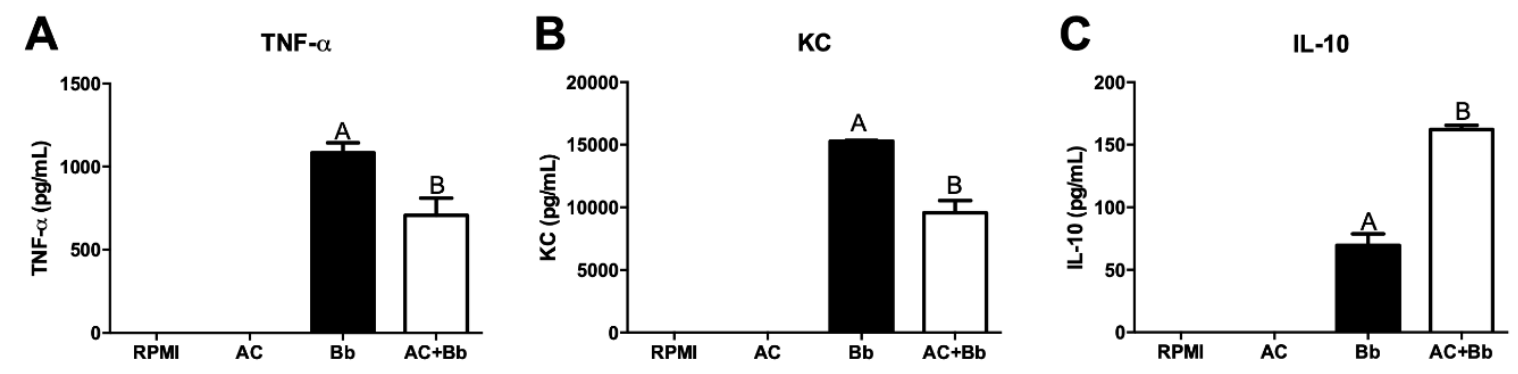

Figure 6: In vitro quantification of cytokines in supernatant of C3H BMDM co-cultured with apoptotic neutrophils.

C3H BMDM were co-cultured with apoptotic neutrophils $(A C)$ and $B$. burgdorferi $(\mathrm{Bb})$ for 24 hours and cytokine levels of TNF- $\alpha(A), K C(B)$, and IL-10 (C) were quantified by ELISA. $n=3$ per group. Bars with different letters are significantly different from each other at $p<0.05$ level. 

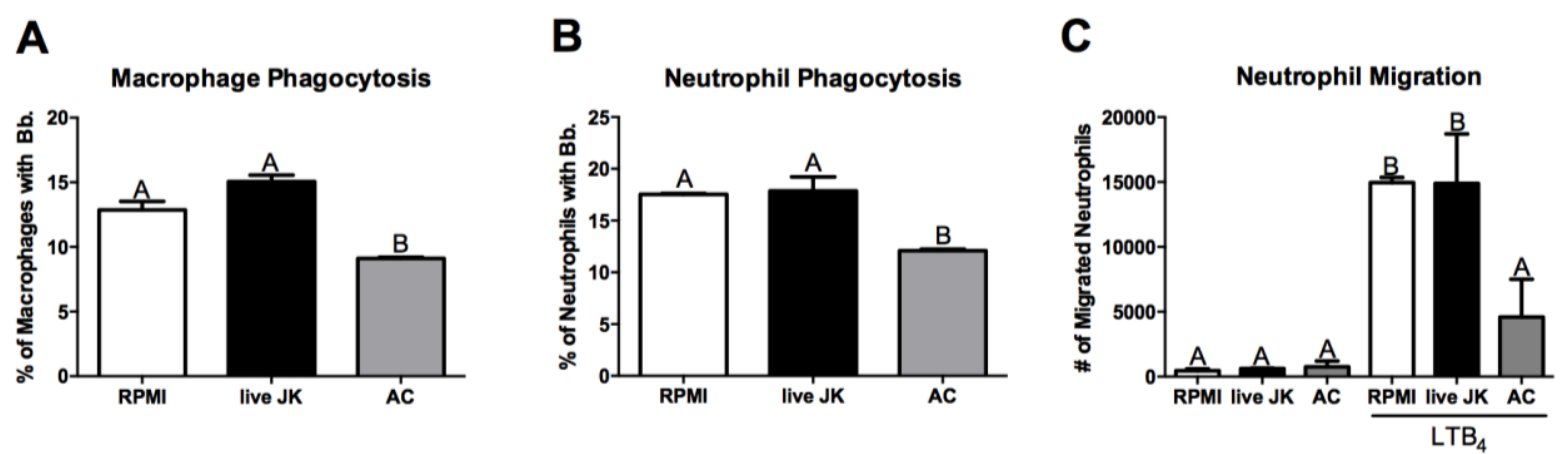

Figure 7: In vitro AC modulation of bacterial clearance and neutrophil migration to $\mathrm{LTB}_{4}$.

$\mathrm{C} 3 \mathrm{H}$ BMDM $(\mathrm{A})$ or $\mathrm{BMN}(\mathrm{B})$ were co-cultured with $\mathrm{AC}$ for 1 hour prior to the addition of GFP B. burgdorferi. After 4 hours (A) or 2 hours (B), flow cytometry was used to determine the number of $\mathrm{GFP}^{+}$cells. $\mathrm{C} 3 \mathrm{H}$ BMN were co-cultured with $\mathrm{AC}$ for 2 hours prior to the migration to $\mathrm{LTB}_{4}$ for an additional 2 hours (C). $n=3$ per group. Bars with different letters are significantly different from each other at the $p<0.05$ level. 
role in neutrophil recruitment during Lyme arthritis [3]. Neutrophil incubation with AC significantly decreased the number of neutrophils migrating to $\mathrm{LTB}_{4}$ compared with controls (Figure 7C). These data show that $A C$ can also alter macrophage and neutrophil functions in vitro in the presence of $B$. burgdorferi.

\section{Apoptotic cells increase PPAR- $\psi$ in vitro and in vivo during B. burgdorferi} infection

The nuclear transcription factor PPAR- $\gamma$ is a known negative regulator of macrophage activation and plays an important role in AC-mediated antiinflammatory processes $[5,116]$. We therefore wanted to determine if PPAR- $\nu$ was being upregulated by $A C$ in our model. We first measured PPAR- - mRNA levels in BMDM cultures alone or in the presence of AC (Figure 8A). Addition of AC alone had no effect on macrophage PPAR-y transcription levels. Activation of BMDM by co-culture with $B$. burgdorferi alone increased PPAR- $ү$ mRNA levels by 2-fold, but this was not statistically significant compared with BMDM alone. Addition of both $B$. burgdorferi and AC, however, significantly increased macrophage PPAR-y transcription levels in vitro by almost 5 -fold (Figure $8 \mathrm{~A})$. We then determined if PPAR-y expression within the ankle joint increased in vivo during $B$. burgdorferi infection following the administration of AC. Administration of $\mathrm{AC}$ directly into the tibio-tarsal joint at 13 days pi significantly increased PPAR$\checkmark$ levels on 14 days pi as measured by western blot (Figure 8B, 8C). These data indicate that PPAR- $\gamma$ is upregulated when AC are cleared during $B$. burgdorferi 

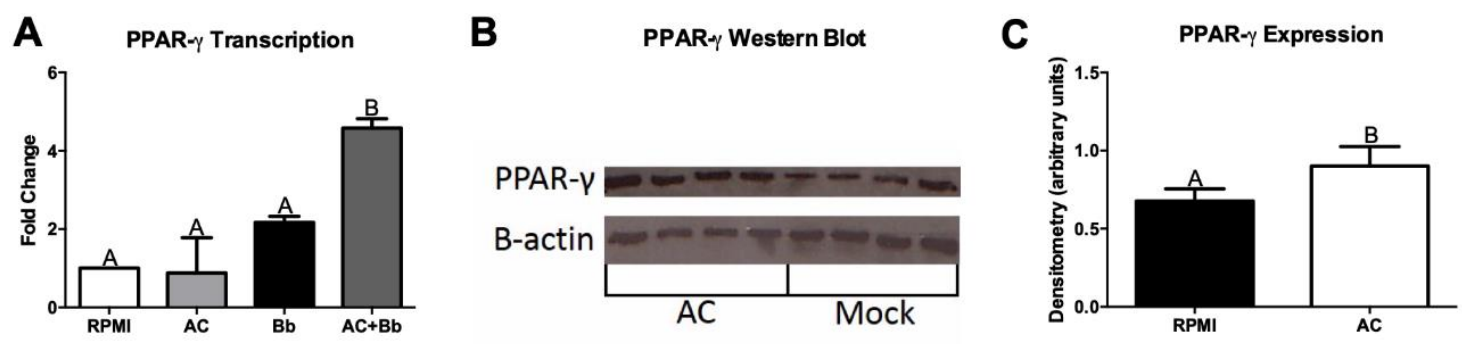

Figure 8: AC induction of PPAR-Y in BMDM and C3H infected mouse joints.

C3H BMDM were co-cultured with AC and/or $B$. burgdorferi for 24 hours, and PPAR-y transcription was determined by real-time PCR (A). $n=3$ per group. $\mathrm{C} 3 \mathrm{H}$ mice were infected with $1 \times 10^{5} \mathrm{~B}$. burgdorferi and given apoptotic Jurkat cells or RPMI 1640 (mock) on day 13 pi. Mice were sacrificed 24 hours later, and PPAR$Y$ expression in the ankle joints was determined via western blot $(B)$ and densitometry (C). $n=4$ per group. Bars with different letters are significantly different from each other at the $p<0.05$ level. 
infection and could possibly explain how AC contribute to inflammation resolution.

\section{The PPAR- $\gamma$ agonist rosiglitazone alters macrophage cytokine production and neutrophil migration in vitro}

To further confirm the role of PPAR- $\gamma$ in AC alteration of immune cells, we next determined if using the PPAR- $\gamma$ agonist rosiglitazone would cause a similar alteration in cytokine production of $B$. burgdorferi co-cultured macrophages as AC. B. burgdorferi co-cultured macrophages treated with rosiglitazone had significantly reduced levels of TNF- $\alpha$ (Figure 9A) and KC (Figure 9B) and significantly increased levels of IL-10 (Figure 9C), which were similar to trends shown with AC (Figure $5 \& 6$ ). In contrast, treatment of BMDM with the PPAR-y inhibitor GW 9662 partially but significantly counteracted the effect of AC on macrophage TNF- $\alpha$ and KC production (Figure 9D, 9E) and completely inhibited the AC induction of IL-10 (Figure 9F).

We next investigated the effect of PPAR- $\gamma$ on neutrophil migration and macrophage and neutrophil phagocytosis of $B$. burgdorferi. In contrast to the inhibitory effects of $A C$ on macrophage and neutrophil phagocytosis of $B$. burgdorferi, treatment of BMDM and bone marrow neutrophils (BMN) with rosiglitazone or GW9662 had no effect on their ability to phagocytose $B$. burgdorferi (Figure 10A, 10B, 10E, 10F). These data suggest that $\mathrm{AC}$ induce other signals besides PPAR- $\gamma$ activation, which impacts bacterial phagocytosis. 

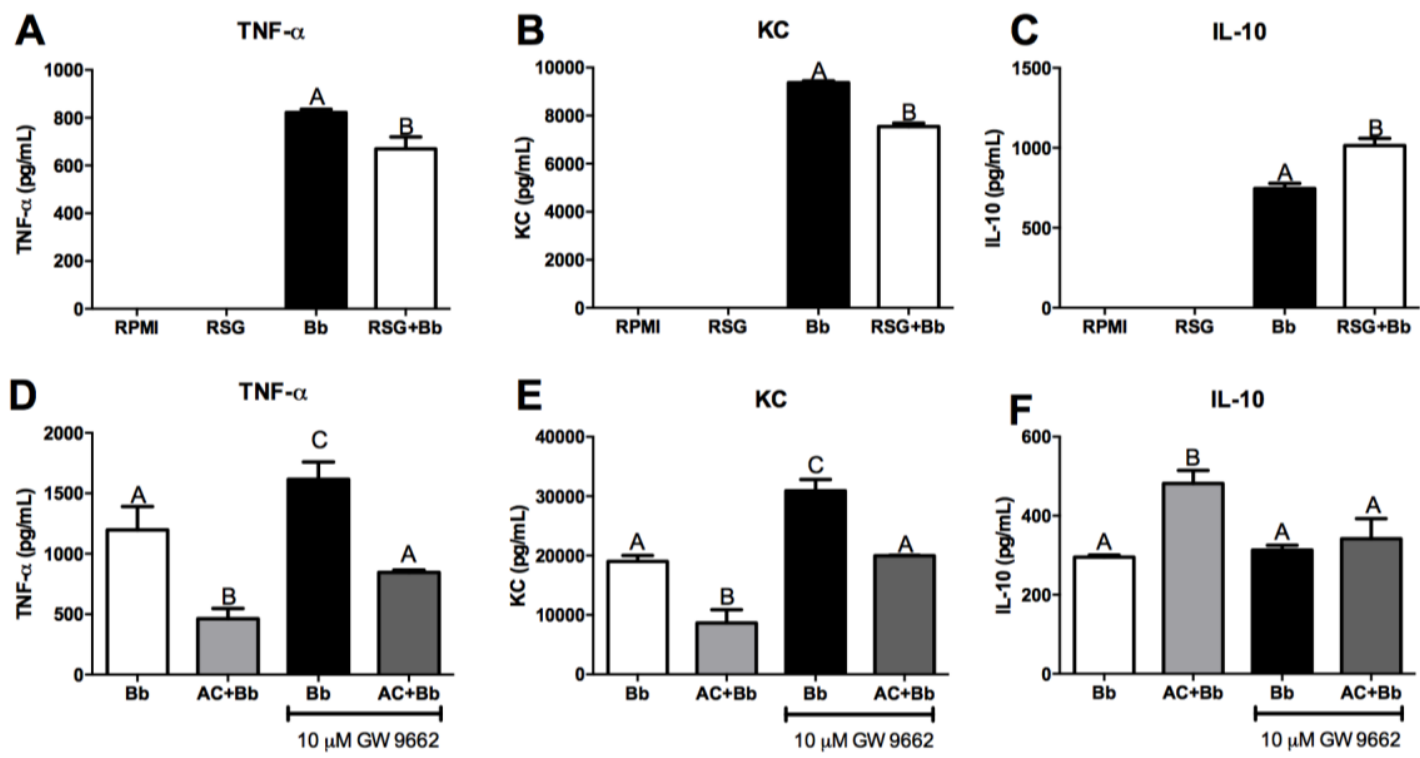

Figure 9: Effect of PPAR-y modulation on BMDM cytokine production.

C3H BMDM were co-cultured with the PPAR-y agonist rosiglitazone (RSG) and/or B. burgdorferi (A-C) or B. burgdorferi and AC and/or the PPAR-y antagonist GW 9662 (D-F) for 24 hours, and levels of TNF- $\alpha$ (A and D), KC (B and $\mathrm{E}$ ), and IL-10 ( $\mathrm{C}$ and $\mathrm{F}$ ) were quantified by ELISA. $n=3$ per group. Bars with different letters are significantly different from each other at the $p<0.05$ level. 

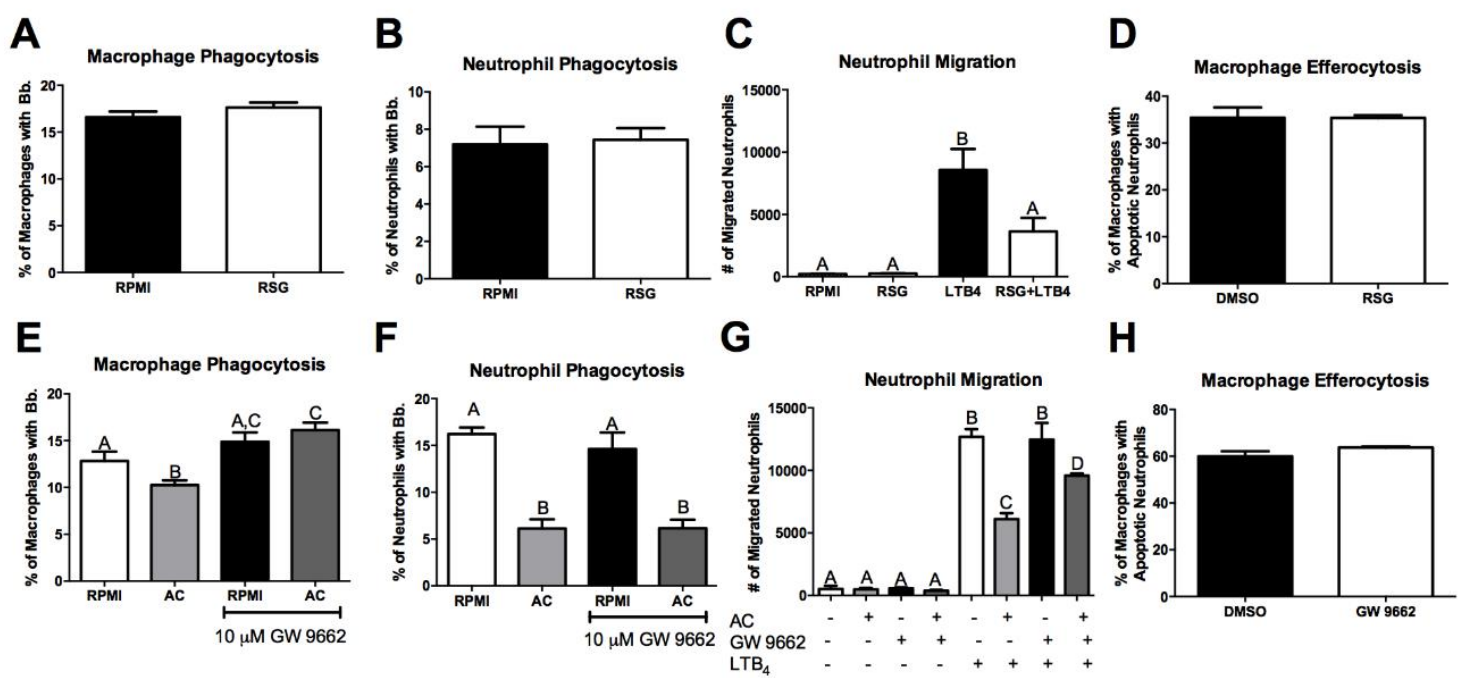

G

H
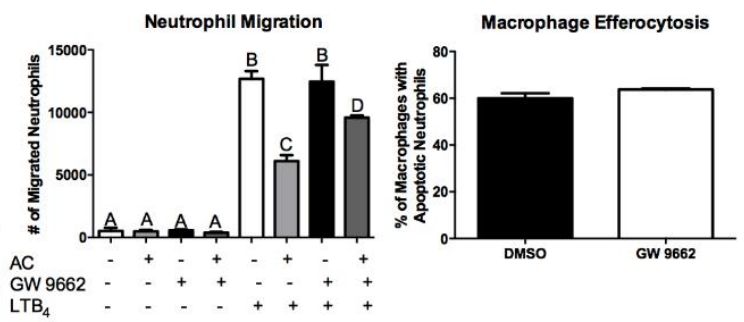

Figure 10: Effect of PPAR-y modulation of bacterial clearance, neutrophil migration to $L_{T B}$, and efferocytosis.

C3H BMDM ( $A$ and $E$ ) or BMN ( $B$ and F) were given rosiglitazone (RSG) or GW 9662 and/or AC for 2 hours (BMN) or 4 hours (BMDM) prior to the addition of GFP $B$. burgdorferi for 4 hours. Flow cytometry was used to determine the number of $\mathrm{GFP}^{+}$cells. $n=3$ per group. C3H BMN were given RSG (C) or GW 9662 and or $A C(G)$ for 2 hours prior to migration to $\mathrm{LTB}_{4}$ for an additional 2 hours. C3H BMDM were treated with RSG (D) or GW $9662(\mathrm{H})$ for 1 hour prior to the addition of CFSE-labeled AC. The percentage of cells containing AC was determined after 4 hours by flow cytometry. $n=3$ per group. Bars with different letters are significantly different from each other at the $p<0.05$ level. 
Similar to the effect of $A C$, treatment of neutrophils with rosiglitazone inhibited their migration toward LTB4 (Figure 10C), and treatment with GW 9662 modulated the effects of AC on neutrophil migration (Figure 10G), suggesting neutrophil recruitment is primarily impacted by PPAR $-\gamma$ mediated mechanisms.

Finally, PPAR- $\gamma$ activation appears to have little effect on efferocytosis, treatment of BMDM with either rosiglitazone or GW 9662 did not alter macrophage clearance of $\mathrm{AC}$ in vitro (Figure 10D, 10H).

\section{Rosiglitazone treatment reduces ankle swelling during Lyme arthritis and suppresses inflammatory cell recruitment}

Finally, we wanted to determine if administration of rosiglitazone in vivo during $B$. burgdorferi infection would impact ankle swelling and immune cell recruitment to the ankle joints. By administering rosiglitazone directly into the ankle joint, as we previously did with $\mathrm{AC}$, ankle swelling was significantly reduced at 21 days pi compared to controls (Figure 11A), similar to treatments with AC. Additionally, both neutrophil (Figure 11B, 11E) and macrophage (Figure 11C, 11F) numbers were significantly reduced in the rosiglitazone-treated mice on both 28 and 35 days pi. These data show that rosiglitazone treatment decreases ankle swelling and inflammatory cell recruitment to the ankle joints during $B$. burgdorferi infection. Unexpectedly, rosiglitazone treatment decreased B. burgdorferi loads in the ankle joints on day 28 pi and significantly lowered spirochete loads on day $35 \mathrm{pi}$ (Figure 11D). This effect was not seen during AC treatment of $B$. 

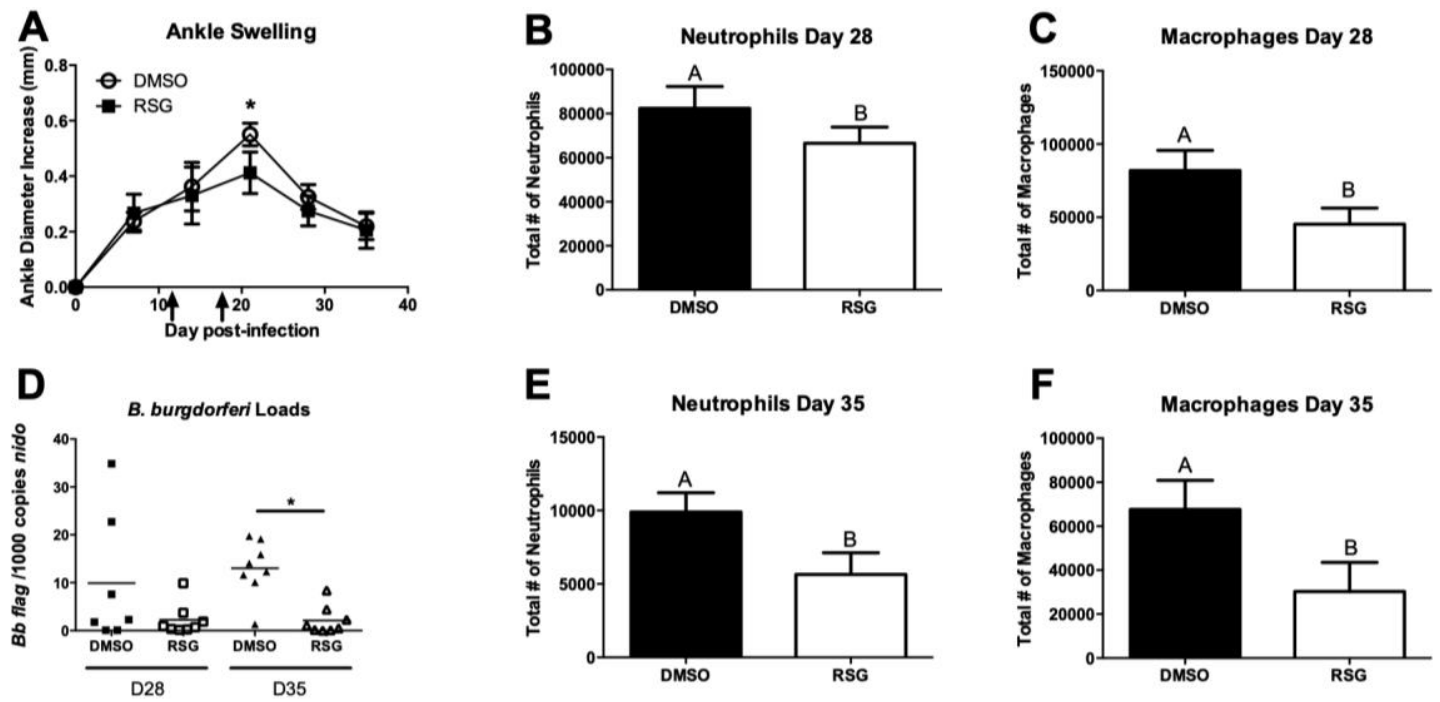

Figure 11: Rosiglitazone treatment of $B$. burgdorferi-infected $\mathrm{C} 3 \mathrm{H}$ mice.

$\mathrm{C} 3 \mathrm{H}$ mice were infected with $1 \times 10^{5} \mathrm{~B}$. burgdorferi and given rosiglitazone or DMSO on days 11 and 18 pi. Ankle swelling was monitored throughout the infection time course $(A)$. Numbers of neutrophils (B and $E$ ) and macrophages (C and $F$ ) within the ankle joints were evaluated on days 28 and 35 pi. B. burgdorferi levels in ankle joints were determined by real-time PCR (D). $n=4$ per group. An asterisk $\left({ }^{*}\right)$ indicates statistical difference from DMSO control at the same time point at the $p<0.05$ level. Bars with different letters are significantly different from each other at $p<0.05$ level. ${ }^{*}=p<0.05$. 
burgdorferi-infected mice (Figure 4C) and suggests that rosiglitazone may induce bacterial clearance by other mechanisms besides PPAR- $\gamma$ activation [121].

\section{Discussion}

The recruitment of neutrophils is known to drive the development of murine Lyme arthritis, however, the mechanisms of how disease resolution occurs remains unclear. The efficient clearance of $\mathrm{AC}$ likely plays a role during arthritis resolution; however, this has never been proven in vivo. The purpose of this study was to determine the role of $A C$ during inflammation resolution in $B$. burgdorferi-infected mice and define a mechanism of how this occurs. As shown with the use of flow cytometry, the number of apoptotic neutrophils and macrophages, the main inflammatory infiltrates, increased within the ankle joints of $B$. burgdorferi-infected mice, peaking during inflammation resolution, around 28 days post infection. Additionally, the injection of $A C$ directly into the ankles of B. burgdorferi-infected mice limited ankle swelling and decreased neutrophil recruitment to the joints without interfering with bacterial clearance. This is a similar phenomenon seen in other models of inflammation [5, 77], and further shows the therapeutic potential of apoptotic cells.

In vitro, the addition of $\mathrm{AC}$ to $\mathrm{BMDM}$ cultures decreased $B$. burgdorferi-induced TNF- $\alpha$ and KC and increased IL-10. In addition, phagocytosis of $B$. burgdorferi and neutrophil migration to $\mathrm{LTB}_{4}$ were inhibited by $\mathrm{AC}$, showing that $\mathrm{AC}$ can modulate the pro-inflammatory nature of macrophages and neutrophils into an 
anti-inflammatory state. The administration of exogenous AC increased PPAR- $\gamma$ expression both in vitro and in vivo during $B$. burgdorferi infection, showcasing a possible mechanism to explain the involvement of $A C$ in inflammation resolution. The PPAR- $\gamma$ agonist, rosiglitazone, elicited similar changes in macrophage cytokine production and neutrophil migration as the use of exogenous AC. The addition of the PPAR- $\gamma$ antagonist GW 9662 abrogated the effects of AC in vitro, further demonstrating the probable role of PPAR- $\gamma$ in this system. Injection of rosiglitazone directly into the tibio-tarsal joints of $B$. burgdorferi-infected mice decreased ankle swelling and immune cell recruitment, similar to the injection of $A C$, further explaining how $A C$ are able to elicit their anti-inflammatory response during $B$. burgdorferi infection. These results suggest clearance of $A C$ plays a role in the resolution of inflammation during experimental Lyme arthritis through the activation of PPAR- $\nu$. Therefore, PPAR- $\gamma$ agonists, such as rosiglitazone, may be useful to ameliorate Lyme arthritis and induce resolution in individuals with posttreatment Lyme disease syndrome. This would be an improvement from current therapeutic strategies that treat the symptoms of the disease, rather than inducing inflammation resolution. 


\section{Chapter 4}

\section{Leukotriene B4 Signaling Through BLT1 Promotes Efferocytosis and Inflammation Resolution During Lyme Arthritis}

\section{Introduction}

The clearance of apoptotic immune cells by macrophages, efferocytosis, is now considered a critical component driving the resolution of inflammation [43]. Neutrophils recruited to the site of infection or injury are programmed to undergo apoptosis to limit their pro-inflammatory responses [122]. The uptake and clearance of apoptotic cells has a profound effect on macrophages, including to down-regulate pro-inflammatory cytokine production and increase their production of anti-inflammatory mediators, such as IL-10 and PGE2 [118]. However, failure to clear apoptotic cells in a timely manner may result in the apoptotic cells undergoing a secondary necrosis and prolonging inflammatory responses [123]. Therefore, a breakdown in apoptosis or efferocytosis can increase inflammation, rather than down-regulate it.

Previous work in our lab has shown in B. burgdorferi-infected mice, 5lipoxygenase (5-LO) $)^{-/-}$mice experienced exacerbated and prolonged arthritis [3]. This was despite normal anti-Borrelia antibody production and spirochete clearance from joint tissues. In contrast, mice deficient in 5-LO activating protein were protected from arthritis development in the collagen-induced arthritis (CIA) model of rheumatoid arthritis [124]. During inflammatory processes, arachidonic 
acid $(A A)$ is released from the membrane by cytosolic phospholipase $A_{2}\left(C P L A_{2}\right)$. AA can then be metabolized by 5-LO into the pro-inflammatory lipids, leukotriene (LT)B4 or the cysteinyl LT [125]. However, 5-LO also produces anti-inflammatory lipids, such as AA derived lipoxins and omega-3 fatty acid derived specialized pro-resolving mediators (SPM), such as D-series resolvins from docosahexaenoic acid (DHA) and E-series from eicosapentaenoic acid (EPA) [126]. The production of lipoxins has been correlated with the resolution of arthritis in the CIA model [127], and the therapeutic treatment of mice with a lipoxin receptor agonist was also able to reduce severity in established CIA [128]. Similarly, treatment of mice with Resolvin $D_{1}$ or $E_{3}$ attenuated arthritis severity in the K/BxN serum-transfer model $[129,130]$. These results showcase the complex nature of bioactive lipid regulation of inflammatory responses and demonstrate that 5-LO metabolites have both pro-inflammatory and pro-resolution effects on inflammation.

Previous work demonstrated a defect in efferocytosis by 5-LO-deficient macrophages which correlated with nonresolution during Lyme arthritis [3]. In this study, we focus on the role of the 5-LO metabolite, $\mathrm{LTB}_{4}$, on Lyme arthritis development and resolution through the use of $\mathrm{BLT1}^{-/}$mice. While $\mathrm{LTB}_{4}$ has two receptors, BLT1 being the high affinity and $B L T 2$ being the low affinity, the focus of this study will be on BLT1, because it is only located on leukocyte subsets while BLT2 is more constitutively expressed [131]. This approach has the 
advantage of leaving other 5-LO-mediated pathways intact, especially those important for inflammation resolution [132].

\section{Results}

\section{Assessment of $B$. burgdorferi infection of $B L T 1^{-/-}$mice}

We have previously reported that infection of mice deficient in 5-LO with $B$. burgdorferi led to exacerbated development of experimental Lyme arthritis and a deficiency of arthritis resolution and prolonged disease [3]. Since 5-LO is responsible for the production of several pro- and anti-inflammatory mediators, we decided to first focus on the role of $\mathrm{LTB}_{4}$ in mediating this response since we have previously shown its expression in the ankle joints during $B$. burgdorferi infection [3]. C3H WT and C3H BLT1 $1^{-/}$mice were infected with $1 \times 10^{5} \mathrm{~B}$. burgdorferi and development of arthritis was followed by monitoring ankle swelling on a weekly basis. During the early part of infection, BLT1/- mice developed ankle swelling similar to WT mice (Figure 12A) However, at later time points, resolution of joint swelling in the $\mathrm{BLT1} 1^{-/}$mice appeared to lag behind the WT mice, and at day 35 pi was significantly higher before returning to baseline levels on day 42 pi. Lack of BLT1 signaling had no effect on spirochete clearance from joint tissues indicating host defense remained intact (Figure 12B). However, ankle swelling is not always indicative of the underlying inflammatory response. Assessment of $\mathrm{H} \& \mathrm{E}$ stained sections from infected joints on day 14 pi revealed relatively equal levels of inflammatory infiltrates at this time point (Figure 12C). However, by day $35 \mathrm{pi}$, a time when WT were undergoing arthritis resolution, 

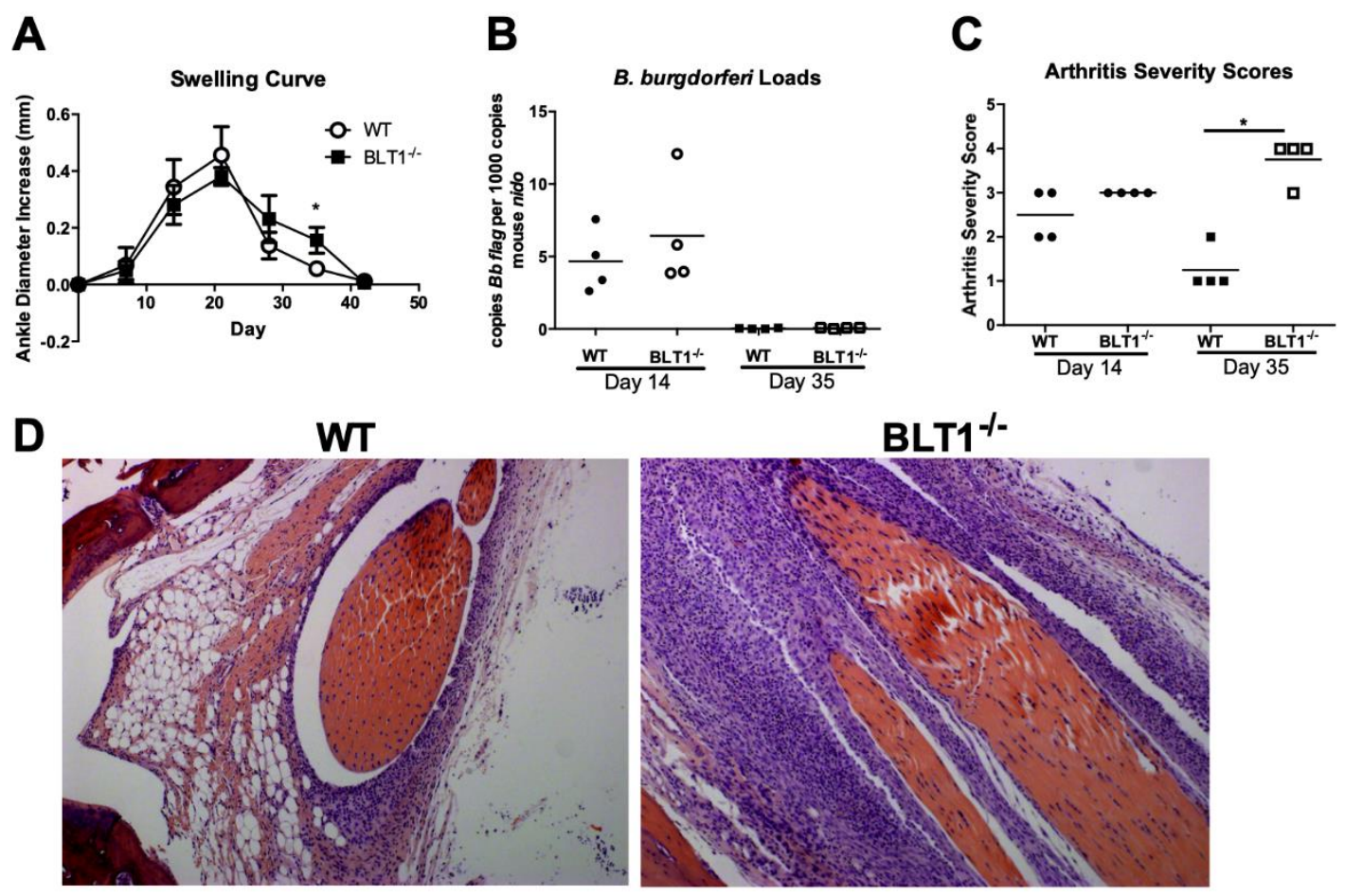

Figure 12: Ankle swelling, B. burgdorferi loads and arthritis severity in B. burgdorferi-infected C3H WT and BLT ${ }^{-/-}$mice.

$\mathrm{C} 3 \mathrm{H} \mathrm{WT}$ and $\mathrm{BLT1} 1^{-/-}$mice were infected with $1 \times 10^{5}$ B. burgdorferi. (A) Ankle swelling was monitored weekly throughout infection. (B) Quantification of $B$. burgdorferi loads of the ankle joints were assessed at indicated days postinfection. (C) Arthritis scores were determined by histological analysis of the ankle joints via $\mathrm{H} \& \mathrm{E}$ staining on days 14 and 35 post-infection. (D)

Representative H\&E stained joint sections on day 35 post-infection. Magnification $40 \mathrm{x} . \mathrm{n}=4$ per group. ${ }^{*}$ indicates significance at $P<0.05$ level. 
ankle joints from $\mathrm{BLT1}^{-/}$mice exhibited severe inflammation and high arthritis severity scores. This is exemplified by representative images of inflammation around the cranial tendon in the joint, which is mild in the WT mice and quite severe in the $\mathrm{BLT1}^{-/}$mice (Figure 12D). These results demonstrate the lack of $\mathrm{LTB}_{4}$ signaling through BLT1 has little effect on the development of experimental Lyme arthritis, but results in a lack of normal arthritis resolution and chronic inflammation.

\section{Assessment of joint cellular infiltrate in WT and $B L T 1^{-/-}$mice during $B$. burgdorferi infection}

Infection of $\mathrm{BLT1} 1^{-/}$mice with $B$. burgdorferi resulted in the normal development of Lyme arthritis with a failure to undergo normal arthritis resolution and an exacerbation of disease severity and duration. We next used flow cytometry to compare joint cellular infiltrates between WT and BLT1/- mice. Mice were infected as before, and joints were harvested on days 14, 28, and 35 pi and cellular infiltrates isolated for flow analysis. On day $14 \mathrm{pi}$, a time point when arthritis severity scores were similar between $\mathrm{WT}$ and $\mathrm{BLT} 1^{-/-}$mice, total neutrophil numbers in the joints were similar although there was a trend for higher numbers in the BLT1 ${ }^{-/}$mice (Figure $13 \mathrm{~A}$ ). However, by day $28 \mathrm{pi}$, a time point when arthritis resolution is beginning to occur in WT mice, BLT1/- have significantly higher total neutrophil numbers in their joints compared to WT joints (Figure 13B). This difference was even greater by day 35 pi (Figure 13C). Similarly, the 

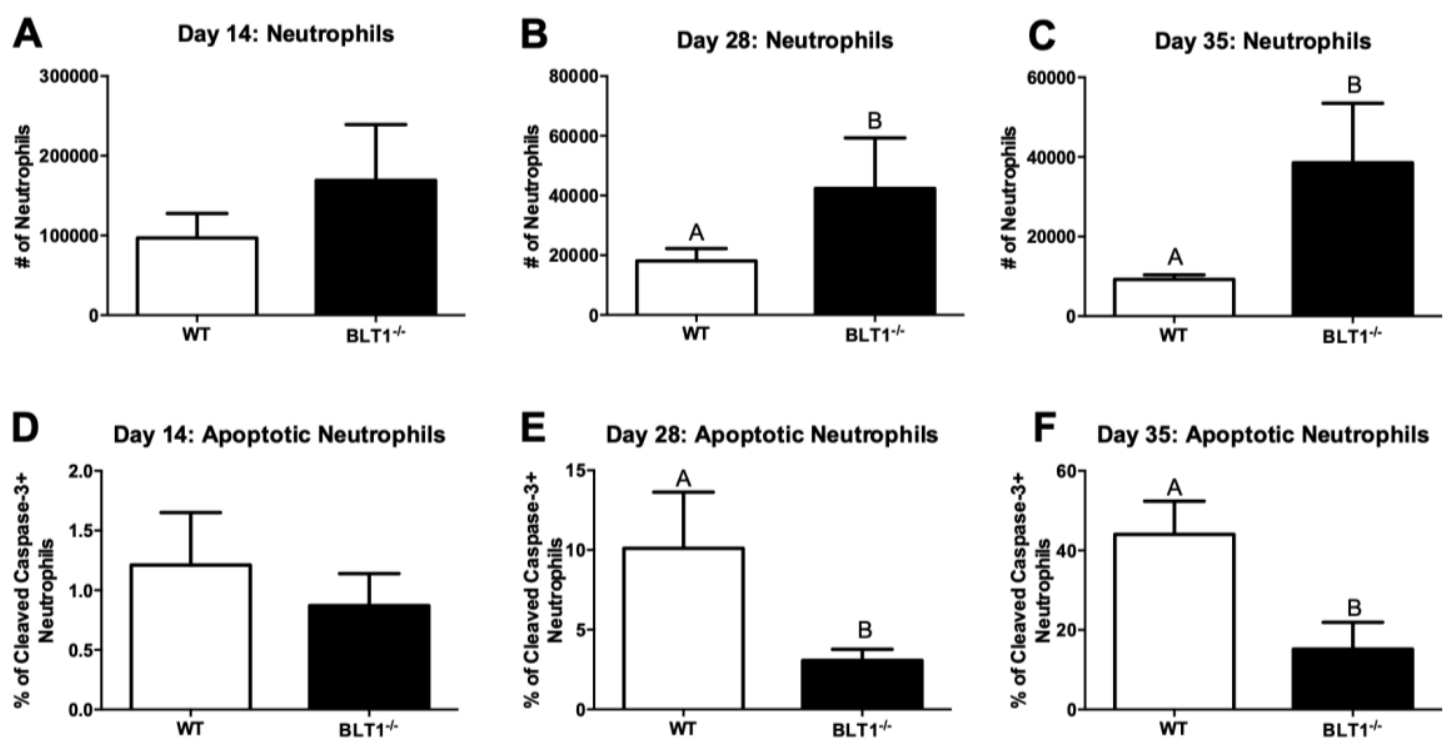

Figure 13: Quantification of neutrophils within the ankle joints of $B$. burgdorferi-infected WT and BLT1 ${ }^{-/-}$mice.

$\mathrm{C} 3 \mathrm{H} \mathrm{WT}$ and $\mathrm{BLT1} 1^{--}$mice were infected with $1 \times 10^{5} \mathrm{~B}$. burgdorferi and sacrificed at indicated days for flow cytometry analysis. Total number of neutrophils (A-C) and the percentage of cleaved-caspase $3^{+}$neutrophils (D-F) were evaluated on days 14,28 , and 35 post-infection. $n=4$ per group. Bars with different letters are significantly different from others a $P<0.05$ level. 
percentage of neutrophils undergoing apoptosis, as measured by cleavedcaspase 3 [133], was similar at day 14 pi, but significantly lower in the BLT1 ${ }^{-/}$ joints on days 28 and 35 (Figure 13D-F). This difference appears to be mediated primarily by differences in neutrophils, as macrophage numbers remain similar until much later during infection (Figure 14A-C). This late increase in macrophages may be due to the prolonged presence of neutrophils in the BLT1/mice. Overall, these data suggest decreased neutrophil apoptosis and clearance may be contributing to the defect in inflammation resolution in $\mathrm{BLT} 1^{-/}$mice.

\section{In vitro characterization of BLT1//- neutrophils}

Neutrophil populations were significantly altered at later time points in $B$. burgdorferi-infected $\mathrm{BLT1}{ }^{-/}$mice. Therefore, we sought to identify intrinsic defects in $\mathrm{BLT1} 1^{-/}$neutrophils. We first determined if $\mathrm{BLT1} 1^{-/}$neutrophils had a defect in their ability to undergo apoptosis. WT and BLT1- BMN were isolated from bone marrow and left as described for 24 hours, and apoptosis was then measured by quantifying cleaved-caspase $3^{+}$cells by flow cytometry. When cultured in media alone, BLT1/- BMN had a significantly lower percentage of apoptotic neutrophils compared with WT BMN (Figure 15A). However, when co-

cultured with B. burgdorferi, the percentage of apoptotic neutrophils was similar between the two groups, but significantly lower than control cultures. Thus, uptake of $B$. burgdorferi appears to delay apoptosis in both WT and BLT1/ BMN. We next examined the ability of $\mathrm{BLT} 1^{-/} \mathrm{BMN}$ to phagocytose $B$. burgdorferi. After 

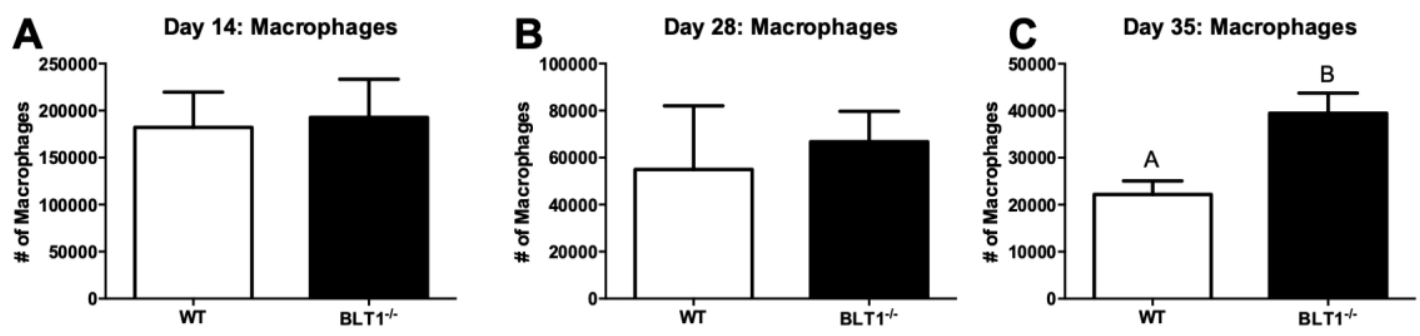

Figure 14: Quantification of macrophages within the ankle joints of $B$. burgdorferi-infected WT and BLT1/- mice.

$\mathrm{C} 3 \mathrm{H} \mathrm{WT}$ and $\mathrm{BLT1} 1^{-/}$mice were infected with $1 \times 10^{5} \mathrm{~B}$. burgdorferi and sacrificed at indicated days for flow cytometry analysis. Total number of macrophages (AC) were evaluated on days 14,28 , and 35 post-infection. $n=4$ per group. Bars with different letters are significantly different from others at $P<0.05$ level. 
A Caspase $-3^{+}$Neutrophils: 24 hours

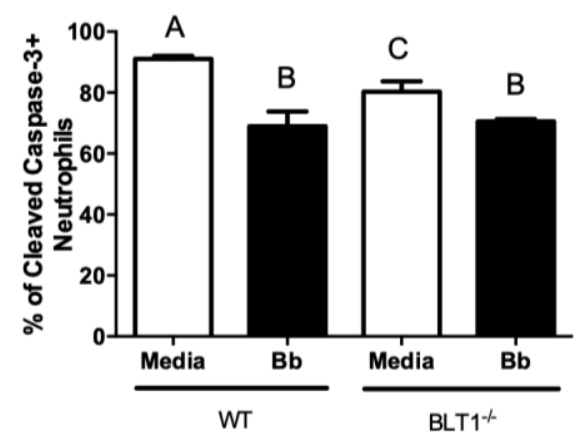

C

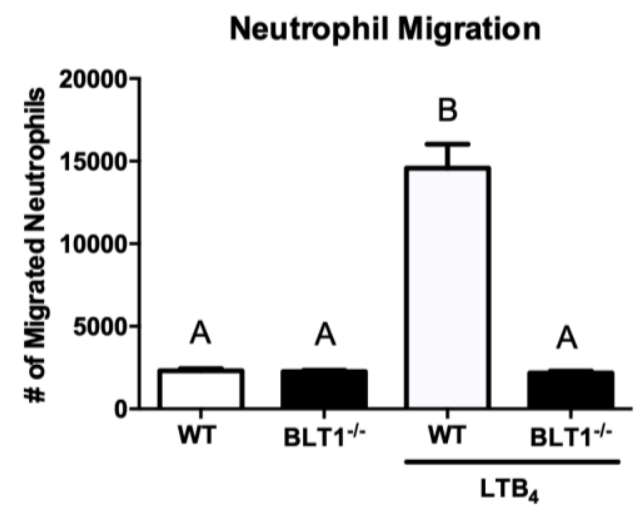

B Neutrophil Phagocytosis

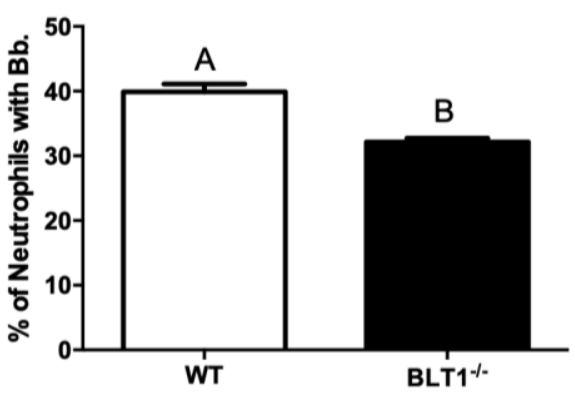

D

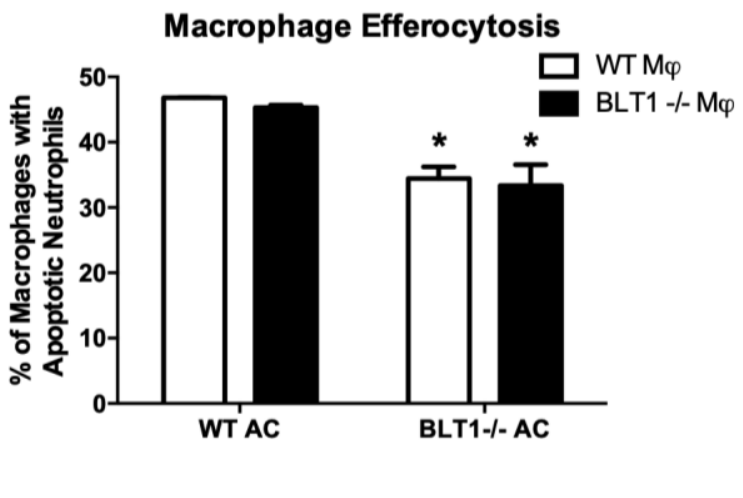

Figure 15: Effector functions of WT and BLT1/-- neutrophils and macrophages in vitro.

(A) $\mathrm{C} 3 \mathrm{H} \mathrm{WT}$ and $\mathrm{BLT1} 1^{-/} \mathrm{BMN}$ were given $B$. burgdorferi and stained for cleavedcaspase 3 after 24 hours for flow cytometry. (B) C3H WT and BLT1- BMN were given GFP-B. burgdorferi for 2 hours with flow cytometry used to determine the number of $\mathrm{GFP}^{+}$neutrophils. (C) $\mathrm{C} 3 \mathrm{H}$ WT and $\mathrm{BLT}^{-1-} \mathrm{BMN}$ were allowed to migrate to $\mathrm{LTB}_{4}$ for 2 hours. Flow cytometry was used to determine the number of neutrophils which had migrated through the trans-well. (D) C3H WT and BLT11- BMDM were given labeled WT or BLT1 $1^{--}$apoptotic BMN for 4 hours. Flow cytometry was used to determine the number of BMDM with labeled apoptotic neutrophils. $n=3$ per group. Bars with different letters are significantly different from others and $P<0.05$ level. * indicates significance at $P<0.05$ level. 
2 hours of co-culture, $\mathrm{BLT1}{ }^{-/} \mathrm{BMN}$ had a significant reduction in $B$. burgdorferi phagocytosis compared to WT BMN (Figure 15B). This is similar to defects in phagocytosis of $B$. burgdorferi previously shown in both $\mathrm{BLT1}^{-/-}[134]$ and $5-\mathrm{LO}^{-/-}$ [3] macrophages.

An important function of $\mathrm{LTB}_{4}$ is the recruitment of neutrophils, however neutrophils seem to be in abundance in $B$. burgdorferi-infected $B L T 1^{--}$mice (Figure 13) and in 5-LO-/- mice [3] which are deficient in LTB4. We confirmed that $\mathrm{BLT1}{ }^{-/} \mathrm{BMN}$ have a significant defect in migration to $\mathrm{LTB} 4$ compare to WT BMN after 2 hours of migration (Figure 15C). Subbarao et al. have previously reported $\mathrm{BLT1} 1^{-/}$neutrophils required $100 \times$ greater $\mathrm{LTB}_{4}$ to migrate at the same rate as WT macrophages [135], therefore other neutrophil chemo-attractants, such as KC, are more likely responsible for neutrophil recruitment during B. burgdorferi infection [2]. The continued presence of neutrophils in the B. burgdorferi-infected joints at late time points in the $\mathrm{BLT1} \%$ mice suggests there may be a defect in clearance of these cells. To determine if $\mathrm{BLT} 1^{-/}$macrophages were defective during efferocytosis, WT or BLT1/- BMDM were co-cultured with either WT or BLT1 ${ }^{-/}$labeled apoptotic BMN for 4 hours. As shown in Figure 15D, WT and $\mathrm{BLT1}{ }^{-1-} \mathrm{BMDM}$ were equally efficient at the clearance of WT apoptotic BMN, however, both WT and BLT1\% macrophages had a significant reduction in the clearance of BLT1/- apoptotic BMN. This demonstrates $\mathrm{BLT1}{ }^{-/} \mathrm{BMDM}$ are competent to clear WT apoptotic BMN, but both WT and BLT1 ${ }^{-/}$BMDM have a defect in the clearance of BLT1 ${ }^{-/}$apoptotic BMN. Therefore, BLT1/- apoptotic 
$\mathrm{BMN}$ are defective in mediating their own clearance by macrophages and may be responsible for the lack of arthritis resolution in the $\mathrm{BLT1} 1^{-/}$mice.

\section{Resolvin $E_{1}$ restores neutrophil apoptosis and B. burgdorferi phagocytosis} in $\mathrm{BLT1}^{-/}$neutrophils, however it does not affect macrophage efferocytosis Resolvin $E_{1}$ is an eicosapentaenoic acid (EPA) derived poly-unsaturated fatty acid (PUFA) with two receptors, BLT1 and ChemR23 [136]. Upon binding, Resolvin $E_{1}$ enhances neutrophil apoptosis [137], reduces neutrophil migration [138], and enhances bacterial phagocytosis and apoptotic cell efferocytosis [139, 140]. We wanted to determine if decreased Resolvin $E_{1}$ signaling was causing the alteration in neutrophil functions in $\mathrm{BLT} 1^{-/}$mice. Administration of exogenous Resolvin $\mathrm{E}_{1}$ slightly enhanced neutrophil apoptosis in $\mathrm{BLT1} 1^{-1-} \mathrm{BMN}$ after 18 hours (Figure 16A) and enhanced B. burgdorferi phagocytosis in both WT and BLT1/neutrophils (Figure 16B). However, Resolvin $E_{1}$ had no effect on efferocytosis in $\mathrm{BLT1}^{-/}$apoptotic neutrophils (Figure 16C). This data indicates while Resolvin $\mathrm{E}_{1}$ aids in restoration of $\mathrm{BLT1}{ }^{-/}$neutrophil apoptosis and $B$. burgdorferi phagocytosis, it does not affect efferocytosis, indicating additional mechanisms may be causing the defect in inflammation resolution in $\mathrm{BLT1} 1^{-/}$mice, most likely responsible for the decreased ability to recognize $\mathrm{BLT1} 1^{-/}$apoptotic neutrophils. 

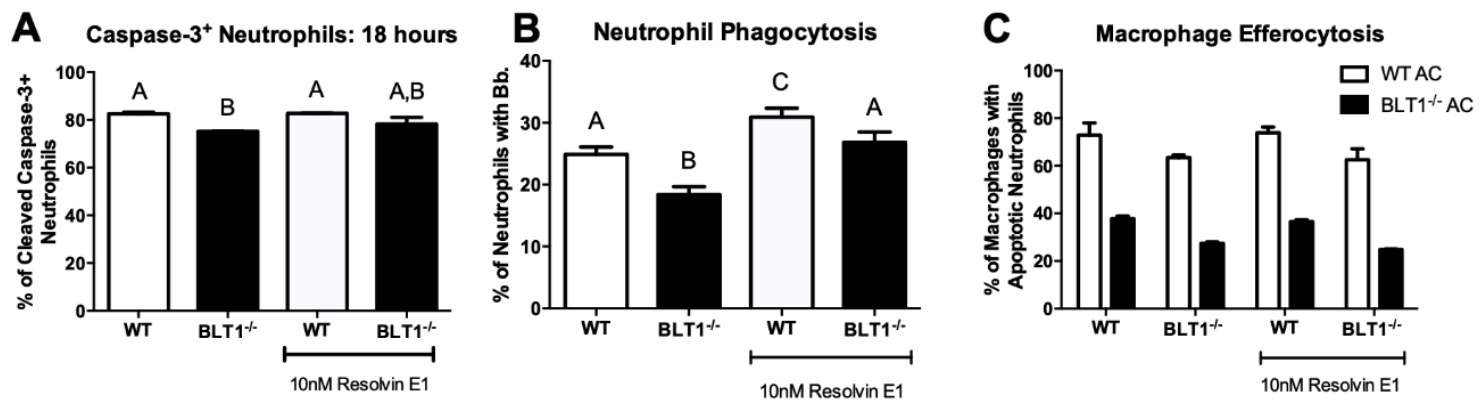

Figure 16: Effector functions of WT and BLT1 ${ }^{-/-}$neutrophils and macrophages in vitro with Resolvin $\mathrm{E}_{1}$.

(A) WT and BLT1\% BMN were given Resolvin E1 and stained for cleaved caspase- 3 after 18 hours for flow cytometry. (B) WT and BLT1/- BMN were given Resolvin $E_{1} 1$ hour prior to the addition of GFP-B. burgdorferi for 2 additional hours. Flow cytometry was used to determine the number of GFP+ neutrophils.

(C) WT and $\mathrm{BLT1} 1^{-/} \mathrm{BMDM}$ were given Resolvin $\mathrm{E}_{1} 3$ hours prior to the addition of labeled WT or $\mathrm{BLT1}^{-/}$apoptotic BMN, which had been cultured in Resolvin $\mathrm{E}_{1}$, for 4 hours. Flow cytometry was used to determine the number of BMDM with a labeled apoptotic neutrophil. $n=3$ per group. Bars with different letters are significantly different from others at a $P<0.05$ level. 


\section{BLT1 ${ }^{-/}$apoptotic BMN have decreased TNF- $\alpha$ production and DISC formation}

While we have shown $\mathrm{BLT1}{ }^{--}$apoptotic $\mathrm{BMN}$ have a defect in recognition by macrophages (Figure 15D), we next determined if $\mathrm{BLT1}{ }^{-}$apoptotic $\mathrm{BMN}$ had altered cytokine production or expression of apoptotic genes compared to WT apoptotic BMN. Freshly isolated BMN from WT or BLT1\% mice were cultured in media for 24 hours, and supernatant was harvested. Apoptotic BLT1 ${ }^{-/}$BMN had significantly decreased TNF- $\alpha$ production compared to WT apoptotic BMN (Figure 17A), however, quantification of KC (Figure 17B) and IL-10 (Figure 17C) showed no difference between $\mathrm{BLT1}{ }^{-/}$and WT apoptotic BMN.

In addition, we were interested in defining changes in the expression of genes associated with apoptosis in BLT1/ apoptotic BMN. To do this we used the apoptosis profiler PCR array which measures the expression of 84 key genes involved in apoptosis. WT and BLT1/ BMN were cultured in media for 24 hours. Cells were harvested and mRNA extracted, cDNA made and normalized to control. Of the 84 genes tested (Appendix 1) 14 were significantly downregulated in expression, indicated by a greater than 2 -fold difference in the $\mathrm{BLT} 1^{-1}$ compared to WT BMN (Table 1). Five of the genes downregulated in BLT1 ${ }^{-/}$ apoptotic BMN, including Cradd, Fadd, Fas, Ltbr, and Traf1, are associated with the death-inducing signaling complex (DISC) [141-144]. This complex is important for TNF-induced apoptosis which is mediated through Fas/FasL interaction [141]. Three additional genes downregulated in BLT1\% apoptotic 

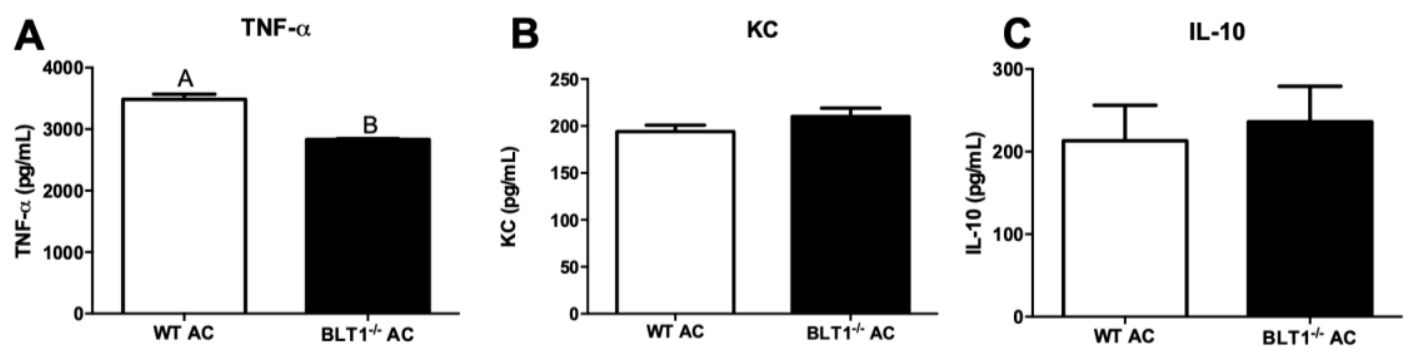

Figure 17: Cytokine production of WT and BLT1 ${ }^{-/}$apoptotic neutrophils in vitro.

WT and BLT1/- BMN were harvested and left for 24 hours. Supernatant was harvested, and the cytokines TNF- $\alpha(\mathrm{A}), \mathrm{KC}(\mathrm{B})$, and IL-10 (C) were measured by Luminex Assay. $\mathrm{n}=3$ per group. Bars with different letters are significantly different from others at $P<0.05$ level. 


\begin{tabular}{|c|c|c|}
\hline Abl1 & C-abl oncogne 1, non-receptor tyrosine kinase & -2.42 \\
\hline Bax & Bcl2-assiciated X-protein & -2.07 \\
\hline Bcl2a1a & B-cell leukemia/lymphoma 2 related protein A1a & -2.16 \\
\hline Bcl211 & Bcl2-like 1 & -2.52 \\
\hline Card10 & Caspase recruitment domain family, member 10 & -2.04 \\
\hline Casp14 & Caspase 14 & -2.16 \\
\hline Casp3 & Caspase 3 & -2.06 \\
\hline Cradd & CASP2 and RIPK1 domain containing adaptor with death & -2.03 \\
& domain & -2.39 \\
\hline Fadd & Fas (TNFRSF6)-assicated via death domain & -2.28 \\
\hline Fas & Fas (TNF receptor superfamily member 6) & -2.40 \\
\hline Igf1r & Insulin-like growth factor I receptor & -2.06 \\
\hline Ltbr & Lymphotoxin B receptor & -2.06 \\
\hline Traf1 & Tnf receptor-associated factor1 & -2.50 \\
\hline Trp53 & Transformation related protein 53 & \\
\hline
\end{tabular}

Table 1: PCR array of apoptosis associated gene expression in BLT1 ${ }^{-/ 2}$ apoptotic neutrophils compared to WT.

cDNA was harvested from WT and BLT1/- apoptotic neutrophils and ran on the Apoptosis $\mathrm{RT}^{2}$ Profiler PCR Array. The genes shown here had a greater than 2fold decrease in expression in the BLT1 ${ }^{-/}$apoptotic neutrophils compared to WT. 
neutrophils, Ab/1, Bax, and Caspase 3, are downstream of the DISC signaling $[145,146]$. We next confirmed decreased expression of the DISC components and downstream effectors in BLT1/- apoptotic BMN compared to WT both in vitro and in vivo by using flow cytometry (Figure 18). FasL expression was used to assess DISC formation, while CD36, important for apoptotic cell recognition and efferocytosis, was used to determine downstream effects of DISC formation [141, 147]. Both FasL (Figure 18C) and CD36 (Figure 18D) expression were significantly decreased in BLT1/- neutrophils compared to WT in vivo at day 14 pi. Thus, BLT1 ${ }^{-/}$apoptotic BMN have decreased TNF- $\alpha$ expression and a downregulation of the formation of components and downstream effects of the DISC, which is responsible for TNF-induced apoptosis.

\section{$\mathrm{BLT1}^{-/-}$apoptotic neutrophils have increased cAMP, preventing DISC formation}

We have shown BLT1/- neutrophils have decreased DISC formation, possibly causing a defect in the induction of apoptosis and recognition by phagocytic cells. Battacharjee et al. demonstrated cyclic-AMP (cAMP) prevents TNFinduced apoptosis by inhibiting DISC formation [141]. LTB4 downregulates CAMP via BLT1 signaling [148], and our lab has previously shown LTB4 decreases CAMP in BMDM [134], therefore we wanted to determine if BLT1 ${ }^{--}$apoptotic neutrophils were unable to downregulate CAMP, preventing DISC formation. As shown in Figure 19, BLT1 ${ }^{-/}$apoptotic neutrophils had significantly increased levels of cAMP compared to WT. This data suggests the inability to downregulate 
A

FasL

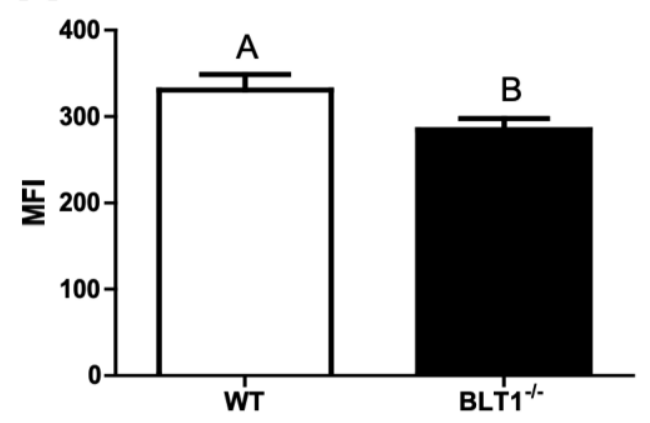

C

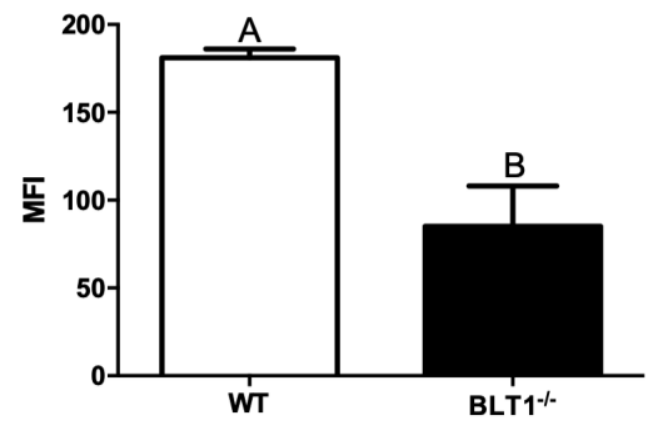

B

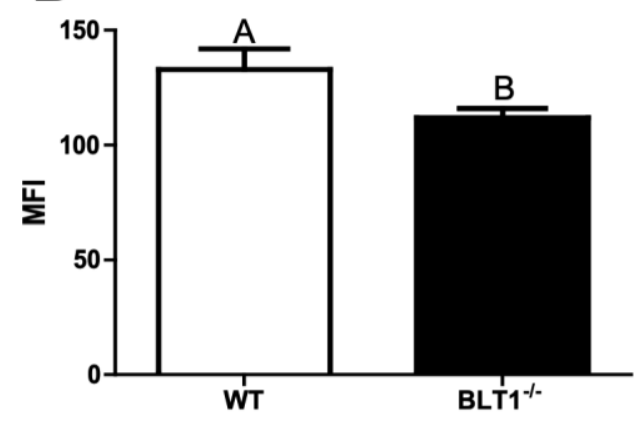

D

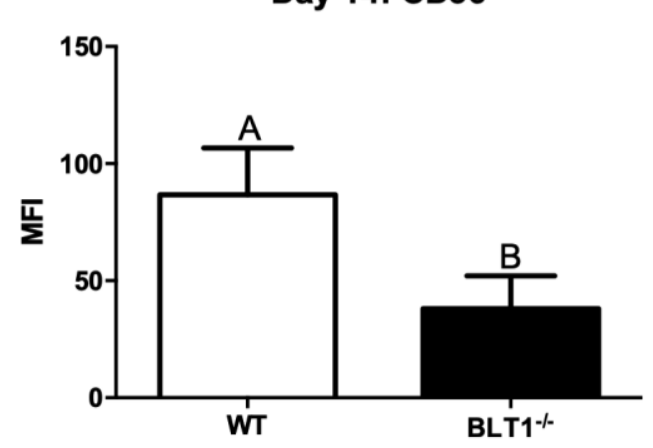

Figure 18: FasL and CD36 expression on WT and BLT1/- apoptotic neutrophils in vitro and $B$. burgdorferi-infected joint neutrophils on day 14 pi in vivo.

WT and BLT1 1 BMN were harvested and left for 24 hours. FasL (A) and CD36 (B) was measured by mean fluorescence intensity (MFI) by flow cytometry. WT and $\mathrm{BLT} 1^{-/}$mice were infected with $1 \times 10^{5} \mathrm{~B}$. burgdorferi and sacrificed at day 14 pi for flow cytometry analysis. FasL (C) and CD36 (D) MFI was measured on CD45.2+, Ly6G+ neutrophils. $n=3$ per group. Bars with different letters are significantly different from others at $P<0.05$ level. 


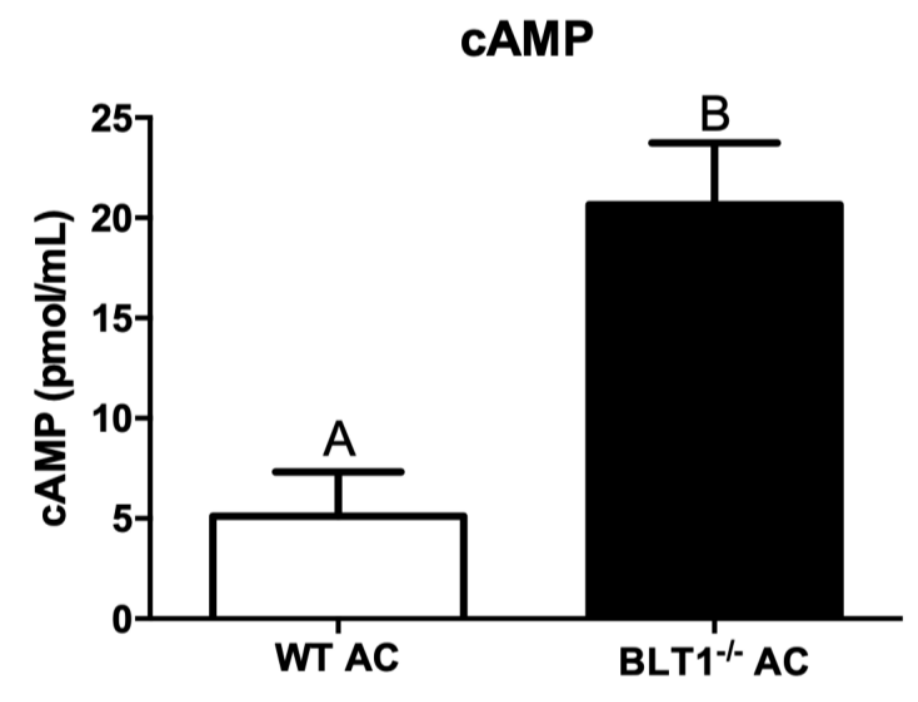

Figure 19: Levels of CAMP in WT and BLT1/-- apoptotic neutrophils.

WT and BLT1/ BMN were harvested and left for 24 hours. Intracellular CAMP was measured by ELISA. $n=3$ per group. Bars with different letters are significantly different from others at $P<0.05$ level. 
CAMP in $\mathrm{BLT1}{ }^{-/}$neutrophils prevents DISC formation, therefore causing decreased apoptotic cell recognition and a defect in efferocytosis leading to prolonged inflammation and a defect in arthritis resolution.

\section{Discussion}

During Lyme arthritis, it is known neutrophil recruitment to the ankle joints drives arthritis development, but the mechanisms involved in arthritis resolution remain unclear. The clearance of apoptotic cells is important during Lyme arthritis resolution, however, if there is a breakdown in apoptosis or efferocytosis, the cells can undergo a secondary necrosis and further contribute to the inflammatory response. Previously, infection of $5-\mathrm{LO}^{-/-}$mice with $B$. burgdorferi resulted in normal arthritis development but had failed arthritis resolution and decreased macrophage efferocytosis [3]. LTB4 is a pro-inflammatory downstream product of 5-LO and signals through BLT1 or BLT2 [131]. BLT1 is the high-affinity receptor found mainly on leukocytes [131], therefore we wanted to determine if signaling through this receptor was responsible for the failure of arthritis resolution in the $5-\mathrm{LO}^{-/-}$mice. Here we show $\mathrm{BLT1} 1^{-/-}$mice infected with $B$. burgdorferi developed arthritis similar to WT mice but failed to undergo arthritis resolution as with $5-\mathrm{LO}^{-/-}$mice. This failure was characterized by increased ankle swelling and prolonged cellular infiltrate in the joints of $\mathrm{BLT1} 1^{--}$mice compare to WT mice. Also similar to $5-\mathrm{LO}^{-/-}$, these mice had similar bacterial loads of $B$. burgdorferi throughout infection as WT mice. Although there was an increased presence of neutrophils in the joints of $\mathrm{BLT1} 1^{-/}$during resolution, there was a 
lower percentage of apoptotic neutrophils suggesting a defect in neutrophil apoptosis and clearance. In vitro, BLT1/- neutrophils had a slight delay in apoptosis and a defect $B$. burgdorferi phagocytosis, along with $\mathrm{BLT} 1^{-/}$apoptotic neutrophils causing a significant decrease in efferocytosis in both WT and BLT1 ${ }^{-}$ macrophages. This suggested the defect associated with $\mathrm{BLT} 1^{-/}$may be the result of a defect in BLT1 ${ }^{-/}$apoptotic cells. Additionally, the administration of Resolvin $\mathrm{E}_{1}$, a pro-resolving mediator known to signal through $\mathrm{BLT1}$, was able to restore apoptosis and B. burgdorferi phagocytosis in BLT1/-neutrophils, however it had no effect on efferocytosis.

As determined by PCR Array and flow cytometry, Death-induced signaling complex (DISC) formation was defective in $\mathrm{BLT1} 1^{-/}$apoptotic neutrophils, which caused decreased CD36 expression, a downstream effector of DISC.

Additionally, the cause of decreased DISC formation was from BLT1 ${ }^{-/}$apoptotic neutrophils inability to downregulate cAMP through $\mathrm{LTB}_{4}$ signaling. These results suggest that in addition to being a primary driver of neutrophil recruitment and inflammation, $\mathrm{LTB}_{4}$ signaling through $\mathrm{BLT1}$ is also an important component of neutrophils apoptosis. Inhibition of this signaling pathway can lead to prolonged inflammation by inhibiting neutrophil apoptosis and clearance and promoting the development of chronic inflammatory disease. 


\section{Chapter 5}

\section{The Effects of Apoptotic Cell Secretions and Activated Apoptotic Cells on Innate Immune Cell Function}

\section{Introduction}

Apoptosis was first thought of as a passive process and was only involved in normal cell turnover, such as recycling of red blood cells and skin cells, however, recent studies have found it has a dynamic role in the down-regulation of proinflammatory responses. Phosphatidylserine (PS) on the outside of the cell is one of the hallmark characteristics of $A C$ and is involved in its recognition and engulfment by phagocytic cells [19]. While recognition of PS is involved in the down-regulation of inflammatory responses of macrophages and dendritic cells through efferocytosis, AC themselves can also secrete factors that also play a role in modulating immune responses to neighboring cells [149]. Several cytokines have been previously shown to be secreted by apoptotic cells, including IL-10 and IL-38, to modulate immune responses [150]. Additionally, the eicosanoid $\mathrm{PGE}_{2}$ is produced by dying cells and acts as an inhibitory danger associated molecular pattern (iDAMP) to prevent cell death associated inflammation [151]. While we and others have shown the presence of AC alters macrophage and neutrophil effector functions $[6,118]$, we next wanted to determine if the secreted products of $A C$ could modulate innate cell function in a similar or partial capacity compared to the presence of the entire AC itself. 
During the immune response to $B$. burgdorferi infection in mice, neutrophils are first recruited to the joints to phagocytose the spirochetes and then undergo apoptosis [6]. Macrophages and other phagocytic cells are recruited for efferocytosis of apoptotic neutrophils containing phagocytosed B. burgdorferi. Therefore, we developed an in vitro system to better replicate in vivo timing of bacterial presence, neutrophil recruitment, and effector function modulation of immune cells after efferocytosis.

Bacterially-activated apoptotic neutrophils have been reported to have various impacts on inflammation and disease. The efferocytosis of apoptotic Escherichia coli-infected dendritic cells promoted high levels of anti-inflammatory cytokine production, while suppressing inflammatory pathways, causing the downregulation of inflammation and promoting resolution [57]. However, Klebsiella pneumoniae infection of neutrophils impaired efferocytosis clearance by modulation of cell host machinery, causing prolonged survival and inflammation [152]. Therefore, neutrophil activation by $B$. burgdorferi could potentially have either positive or negative impact on the immune response and inflammation resolution. Thus, this study will focus on how AC secretions and B. burgdorferiactivated apoptotic neutrophils modulate macrophage and neutrophil functions in vitro. 


\section{Results}

\section{Apoptotic cell supernatant alters macrophage and neutrophil responses in}

vitro

We first wanted to determine how the administration of supernatant from apoptotic Jurkat cells influenced macrophage cytokine production when cocultured with B. burgdorferi. Shown in Figure 20, the administration of these AC to $B$. burgdorferi co-cultured macrophages significantly decreased proinflammatory cytokines TNF- $\alpha$ (Figure 20A) and KC (Figure 20B) and increased anti-inflammatory IL-10 (Figure 20C), as we have previously shown (Figure 5). Additionally, the administration of the AC supernatant caused a similar TNF- $\alpha$ decrease as did the apoptotic Jurkat cells (Figure 20A). However, the AC supernatant had no effect on KC (Figure 20B) or IL-10 (Figure 20C) production, showing the AC supernatant alone could only alter some of the cytokines known to be modulated by AC.

We next wanted to determine if the AC supernatant alone could alter macrophage and neutrophil B. burgdorferi phagocytosis and neutrophil migration in a similar fashion as AC (Figure 7). Both macrophage and neutrophil phagocytosis of $B$. burgdorferi was not affected by the co-culture with live Jurkat cells or Jurkat cell supernatant (Figure 21A, 21B), however, the administration of both apoptotic Jurkat cells and apoptotic Jurkat cell supernatant significantly decreased the percentage of macrophages (Figure 21A) phagocytosing $B$. 

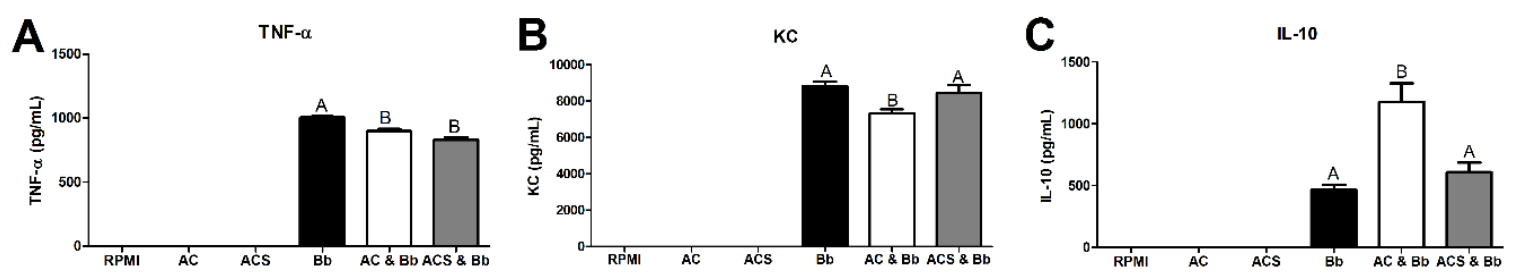

Figure 20: In vitro quantification of cytokines in C3H BMDM supernatant cocultured with $A C$ and $A C$ supernatant.

C3H BMDM were co-cultured with either apoptotic Jurkat cells (AC) or apoptotic Jurkat cell supernatant (ACS) with $B$. burgdorferi for 24 hours, and cytokines levels of TNF- $\alpha(A), K C(B)$, and IL-10 (C) were quantified by ELISA. $n=3$ per group. Bars with different letters are significantly different from each other at the $p<0.05$ level. 

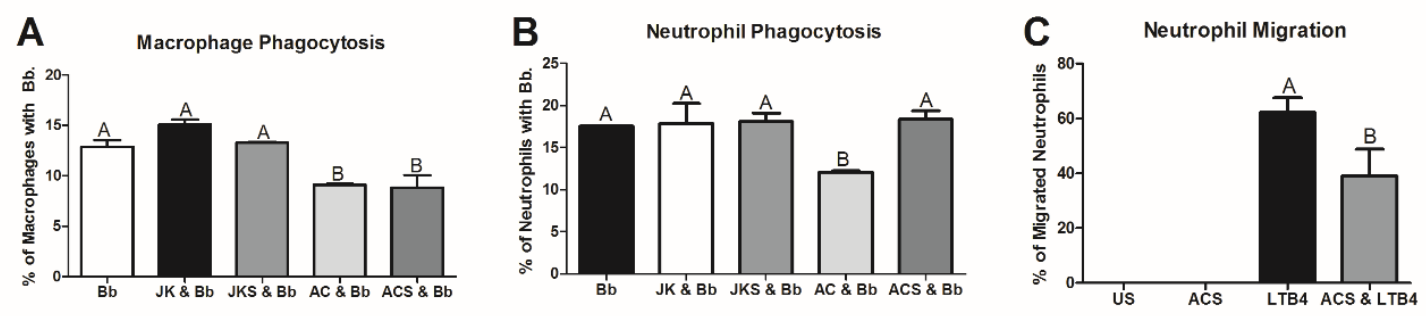

Figure 21: In vitro AC and AC supernatant modulation of bacterial clearance and neutrophil migration to LTB4.

C3H BMDM (A) or BMN (B) were co-cultured with AC or ACS for 1 hour prior to the addition of GFP B. burgdorferi. After 4 hours (A) or 2 hours (B), flow cytometry was used to determine the number of GFP+ ${ }^{+}$cells. $\mathrm{C} 3 \mathrm{H}$ BMN were cocultured with AC or ACS for 2 hours prior to the migration to $\mathrm{LTB}_{4}$ for an additional 2 hours $(C)$. Bars with different letters are significantly different from each other at the $p<0.05$ level. 
burgdorferi, however, only the apoptotic Jurkat cell supernatant altered the percentage of neutrophils phagocytosing B. burgdorferi. (Figure 21B). We also wanted to determine the effect of $A C$ supernatant on neutrophil migration to the chemoattractant $\mathrm{LTB}_{4}$. The percentage of migrated neutrophils to $\mathrm{LTB}_{4}$ was significantly decreased if first pre-incubated with apoptotic Jurkat cell supernatant (Figure 21C), a similar effect as using AC (Figure 7C). These data show AC supernatant can elicit a similar alteration of macrophage and some neutrophil effector functions as AC themselves.

We previously have shown $A C$ administration to $B$. burgdorferi-activated macrophages significantly increased their transcription of PPAR-y in vitro and AC treatment in vivo increased PPAR-y expression within the joints of B. burgdorferiinfected mice (Figure 8). Therefore, we wanted to determine if the secretion of AC would be enough to elicit a similar increase in PPAR-y as did AC administration. As shown in Figure 22, AC supernatant had little effect on PPAR$\mathrm{Y}$ transcription in unstimulated macrophages, however, the co-culture of $\mathrm{AC}$ supernatant in B. burgdorferi-stimulated macrophages caused significantly increased production of PPAR- $\gamma$, similar to AC. This shows the secreted products of the apoptotic cells alone are enough to elicit increased production of PPAR- $\nu$ in $B$. burgdorferi-activated macrophages. 


\section{PPAR- $\gamma$ Transcription}

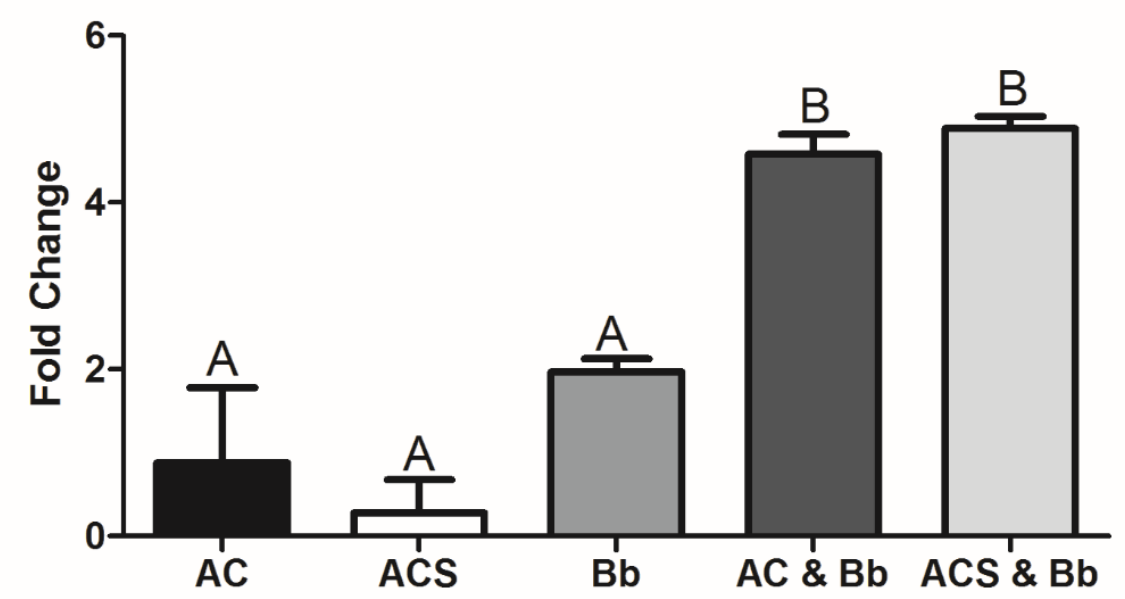

Figure 22: $A C$ and $A C$ supernatant induction of PPAR-Y in BMDM.

C3H BMDM were co-cultured with AC or ACS and/or B. burgdorferi for 24 hours, and PPAR-y transcription was determined by real-time PCR. $n=3$ per group. Bars with different letters are significantly different from each other at the $p<0.05$ level. 

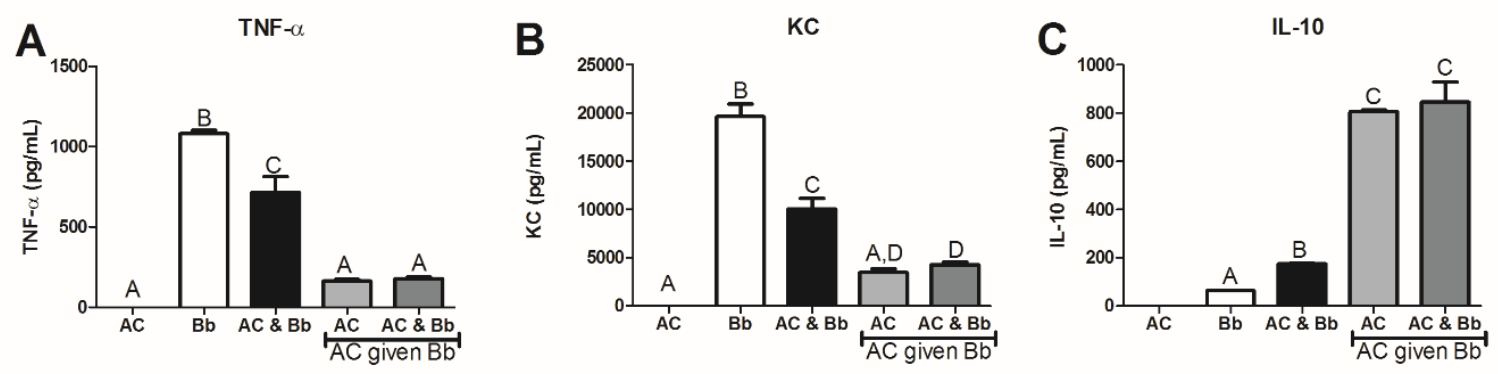

Figure 23: In vitro quantification of cytokines in C3H BMDM co-cultured with apoptotic neutrophils and $B$. burgdorferi-activated apoptotic neutrophils.

C3H BMDM were co-cultured with apoptotic neutrophils (AC) or AC given $B$. burgdorferi prior to apoptosis and $B$. burgdorferi for 24 hours. Cytokine levels of TNF- $\alpha(A), K C(B)$, and IL-10 (C) were quantified by ELISA. $n=3$ per group. Bars with different letters are significantly different from each other at the $p<0.05$ level. 


\section{B. burgdorferi and E. coli activated apoptotic neutrophils alter macrophage cytokine production in vitro}

To further our understanding of the role of $A C$ in eliciting anti-inflammatory responses during $B$. burgdorferi-infection, we developed an in vitro system that would better replicate in vivo conditions. For these experiments, BMN were harvested and co-cultured with B. burgdorferi prior to undergoing apoptosis. After undergoing apoptosis, they were co-cultured with BMDM the same as AC were in previous experiments. We first determined how the activation of neutrophils prior to becoming apoptotic affected their ability to alter macrophage cytokine production. As shown in Figure 23, activating the neutrophils with $B$. burgdorferi caused significantly decreased levels of TNF- $\alpha$ (Figure 23A) and KC (Figure 23B) and increased IL-10 (Figure 23C) compared to B. burgdorferi-activated macrophages or macrophages given non-activated apoptotic neutrophils. While the activated neutrophils caused unstimulated macrophages higher cytokine production than using unstimulated apoptotic cells, the addition of B. burgdorferi to these macrophages caused no further increase in cytokine production.

We then wanted to determine if this alteration of cytokines was a $B$. burgdorferispecific phenomenon or would the enhancement of the effects of bacterial activated neutrophils on macrophage cytokine production still happen if a different bacteria was used to activate the neutrophils. It has been previously shown that E. coli-activated apoptotic dendritic cells were able to downregulate immune responses once efferocytosed [57], so we used E. coli-activated 

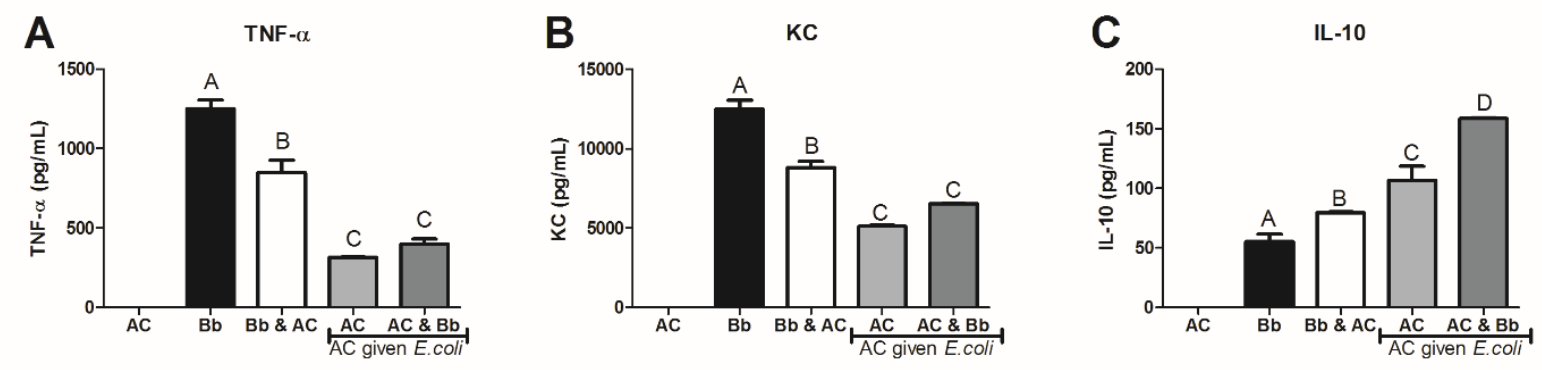

Figure 24: In vitro quantification of cytokines in C3H BMDM co-cultured with apoptotic neutrophils and E. coli-activated apoptotic neutrophils.

C3H BMDM were co-cultured with apoptotic neutrophils (AC) or AC given E. coli prior to apoptosis and $B$. burgdorferi for 24 hours. Cytokine levels of TNF- $\alpha(A)$, $\mathrm{KC}(\mathrm{B})$, and IL-10 (C) were quantified by ELISA. $n=3$ per group. Bars with different letters are significantly different from each other at the $p<0.05$ level. 
neutrophils to compare cytokine alteration in macrophages with $B$. burgdorferiactivated neutrophils. To do this, we gave the neutrophils $E$. coli prior to neutrophil apoptosis, but the macrophages were still activated with $B$. burgdorferi. The E. coli-activated apoptotic neutrophils were able to elicit similar trends in macrophage cytokine alteration as the B. burgdorferi-activated apoptotic neutrophils (Figure 24A-C). These data show if neutrophils are first activated with either B. burgdorferi or E. coli prior to becoming apoptotic, they have an enhanced ability to alter macrophage cytokine production compared to using unstimulated apoptotic neutrophils.

\section{Activated apoptotic neutrophil supernatant alters macrophage cytokine production in vitro}

Our previous data suggested apoptotic Jurkat cell supernatant could elicit changes in macrophage cytokine production, so we determined how the supernatant of $B$. burgdorferi-activated apoptotic neutrophils would affect macrophage cytokine production. The secretions from the B. burgdorferiactivated apoptotic neutrophils worked in a similar fashion as the activated apoptotic neutrophils in enhancing downregulation of TNF- $\alpha$ and $\mathrm{KC}$ and upregulation of IL-10 (Figure 25A-C) production by macrophages. Although KC production was not altered by the administration of apoptotic neutrophil supernatant, the use of $B$. burgdorferi-activated apoptotic neutrophil secreted products were able to significantly decrease the macrophage production of KC,

just as well as B. burgdorferi-activated apoptotic neutrophils (Figure 25B). 

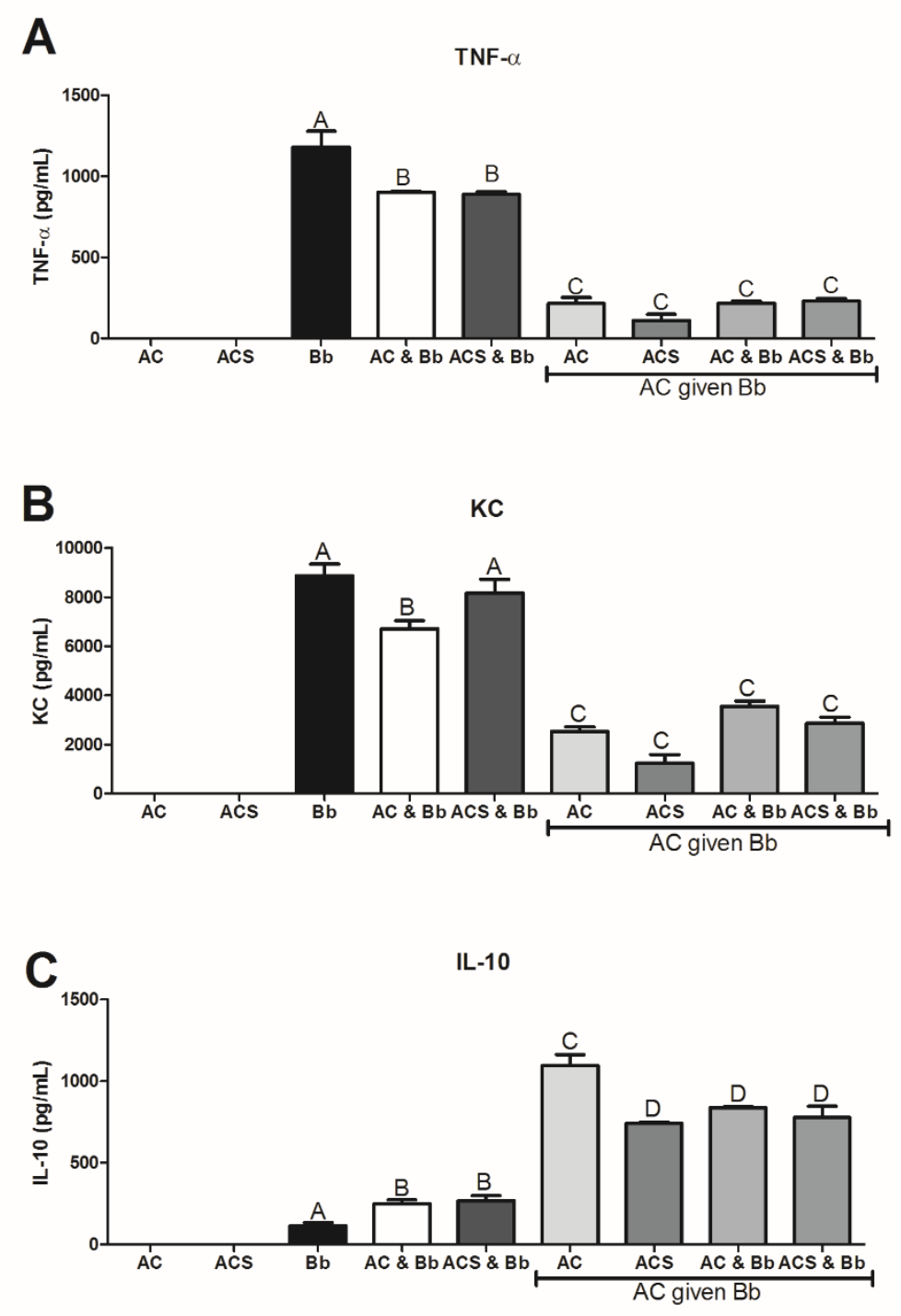

Figure 25: In vitro quantification of cytokines in C3H BMDM co-cultured with apoptotic neutrophil supernatant and B. burgdorferi-activated apoptotic neutrophil supernatant.

C3H BMDM were co-cultured with apoptotic neutrophil supernatant (ACS) or supernatant from $A C$ given $B$. burgdorferi prior to apoptosis and $B$. burgdorferi for 24 hours. Cytokine levels of TNF- $\alpha(A), K C(B)$, and IL-10 (C) were quantified by ELISA. $n=3$ per group. Bars with different letters are significantly different from each other at the $p<0.05$ level. 
Additionally, the supernatant from apoptotic neutrophils contributed to enhanced IL-10 production (Figure 25C), a different phenotype than what was seen with the apoptotic Jurkat cells (Figure 23C), showcasing there might be different products produced by apoptotic Jurkat cells, induced via the extrinsic apoptosis pathway of CD95, compared to apoptotic BMN, induced mainly by intrinsic apoptosis. This shows even the secreted products of activated apoptotic neutrophils have an enhanced ability to alter macrophage cytokine production, just as well as the activated apoptotic neutrophils themselves.

\section{B. burgdorferi-activated apoptotic neutrophils have altered cytokine}

\section{production}

Activated apoptotic neutrophils can alter macrophage cytokine production, however, we have not determined if cytokine production from the apoptotic neutrophils was altered when activated with $B$. burgdorferi prior to induction of apoptosis. As measured by Luminex, the cytokines KC, IL-10, and IL-6 were significantly higher in the $B$. burgdorferi-activated apoptotic neutrophils compared to unstimulated apoptotic neutrophils (Figure 26A, 26C, and 26E) with no change in MCP-1 levels (Figure 26D). Additionally, the only cytokine measured that was significantly decreased in the activated apoptotic neutrophils was TNF- $\alpha$ (Figure 26B). Although KC production was significantly increased in the activated apoptotic neutrophils, this did not translate to increased $\mathrm{KC}$ production in $B$. burgdorferi co-cultured macrophages (Figure 23B). This data shows even though activated apoptotic neutrophils make higher levels of anti-inflammatory cytokines 

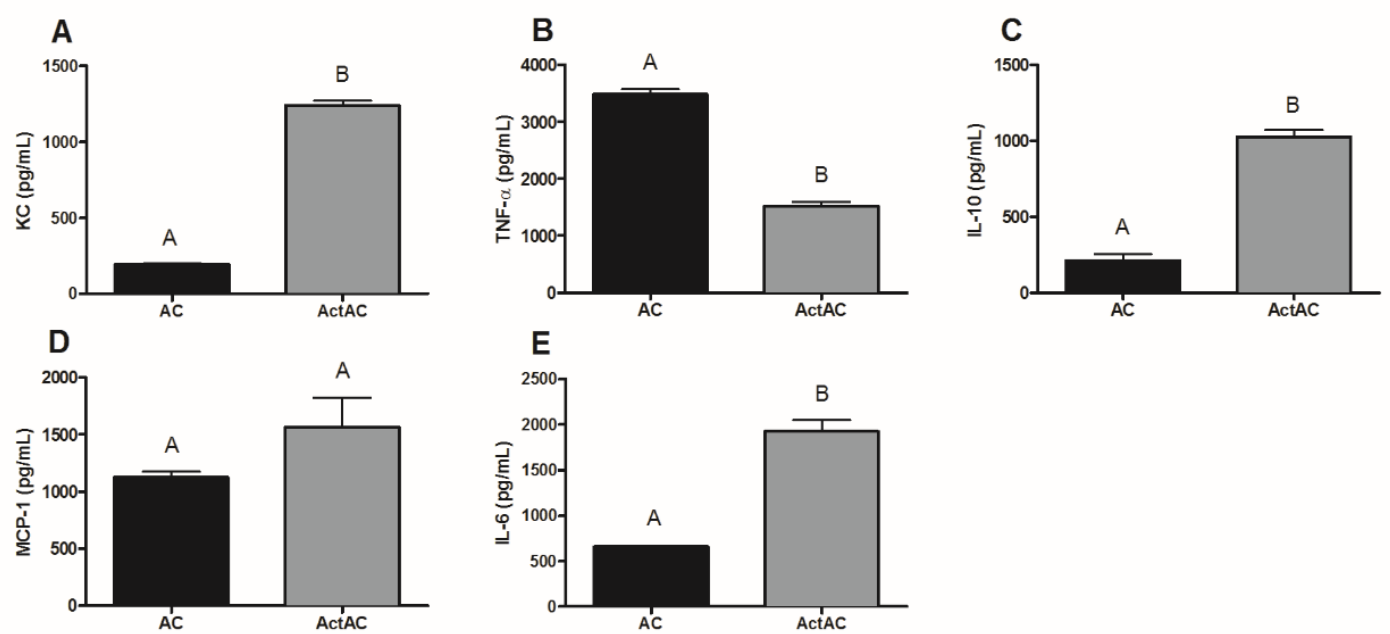

Figure 26: In vitro quantification of apoptotic neutrophil and B. burgdorferiactivated apoptotic neutrophil supernatant.

$\mathrm{C} 3 \mathrm{H}$ BMN were co-cultured with (ActAC) or without (AC) B. burgdorferi prior to apoptosis for 24 hours. Cytokine levels in the supernatant of KC (A), TNF- $\alpha(B)$, IL-10 (C), MCP-1 (D) and IL-6 (E) were quantified by Luminex Assay. $n=2$ per group. Bars with different letters are significantly different from each other at the $p<0.05$ level. 
like IL-10, they also produce significantly higher levels of pro-inflammatory cytokines, however this does not contribute to increased inflammatory cytokine production by macrophages (Figure 23). Additionally, the only cytokine measured which decreased in the activated apoptotic neutrophils was TNF- $\alpha$, suggesting TNF- $\alpha$ regulated inflammatory pathways may be causing the effect of the activated apoptotic neutrophils.

\section{B. burgdorferi apoptotic neutrophils are efferocytosed at the same rate as unstimulated neutrophils}

We have shown AC can downregulate responses in both macrophages and neutrophils (Figure 7) but have yet to determine how activated apoptotic neutrophils affect these functions. We next wanted to determine if activated apoptotic neutrophils were phagocytosed by macrophages at a different rate than unstimulated apoptotic neutrophils. As shown in Figure 27, the same percentage of macrophages had efferocytosed unstimulated apoptotic neutrophils and $B$. burgdorferi-activated apoptotic neutrophils. This shows even though activated apoptotic neutrophils are efferocytosed at the same rate as unstimulated apoptotic neutrophils, they still have a greater impact on macrophage cytokine production.

\section{Discussion}

While we know the clearance of $A C$ can downregulate immune responses, determining what components of the $A C$ are needed to elicit these responses will 


\section{Macrophage Efferocytosis}

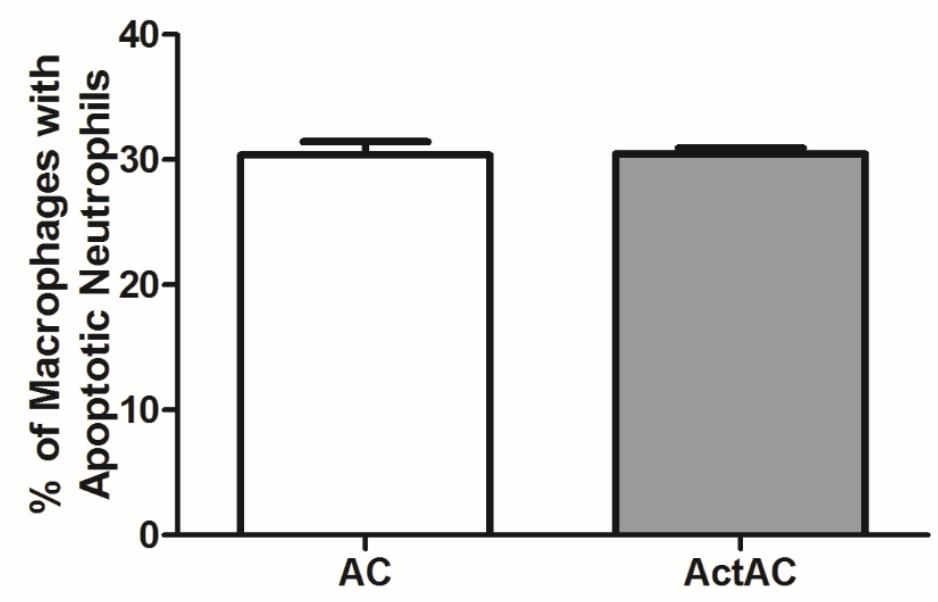

Figure 27: Effect of B. burgdorferi-activated apoptotic neutrophils on macrophage efferocytosis.

C3H BMDM were given CFSE-labeled apoptotic neutrophils cells $(A C)$ or neutrophils activated with $B$. burgdorferi prior to apoptosis (ActAC) for 4 hours. The percentage of macrophages containing $A C$ was determined by flow cytometry. $n=3$ per group. Bars with different letters are significantly different from each other at the $p<0.05$ level. 
aid in a better understanding of how AC function during inflammation resolution. Some groups have used PS coated beads or liposomes in lieu of AC [153, 154], however, others have shown $A C$ can secrete factors that play a role in the downregulation of immune responses $[150,151]$. This shows not just the surface exposure of PS should be used to replicate the presence of $A C$, because $A C$ are actively making and secreting cytokines, eicosanoids, and other factors as well.

We therefore wanted to determine how the secretion of $A C$ affected macrophage and neutrophil functions in vitro when co-cultured with $B$. burgdorferi. As shown here, the secretions of the apoptotic Jurkat cells were able to alter macrophage production of TNF- $\alpha$ as well as the apoptotic cells themselves, however the other cytokines measured, KC and IL-10, were not affected. This was different from using the supernatants of the apoptotic neutrophils, in which IL-10 was also altered. The discrepancies between the two types of apoptotic cell supernatant suggests apoptotic Jurkat cells and apoptotic BMN may secrete different factors. Additionally, macrophage and neutrophil phagocytosis of $B$. burgdorferi and neutrophil migration was significantly reduced when given the $A C$ secretions. Also, the pro-resolution transcription factor PPAR- $\gamma$ was also significantly increased in macrophages treated with the AC supernatant alone. This data suggests cells may not require the actual uptake of $A C$ for their immune responses to be down-regulated by $A C$ but may be influenced by $A C$ in the local environment. 
We also wanted to determine what effects activation of neutrophils prior to their apoptosis would have on their ability to alter macrophage functions. The administration of both B. burgdorferi- and E. coli-activated apoptotic neutrophils significantly decreased pro-inflammatory and increased anti-inflammatory cytokines much greater than using unstimulated apoptotic neutrophils. The supernatant of the $B$. burgdorferi-activated apoptotic neutrophils also was able to elicit a similar response. However, this increased ability to alter macrophage function was not due to decreased pro-inflammatory cytokine production by the activated apoptotic neutrophils, as they produced significantly higher levels of both pro- and anti-inflammatory cytokines compared to unstimulated apoptotic neutrophils of the cytokines we measured. The only cytokine measured that was significantly lower in the activated apoptotic neutrophils was TNF- $\alpha$, suggesting TNF- $\alpha$ down-regulating pathways may explain why the activated apoptotic neutrophils elicit a much stronger alteration in cytokines by the macrophages. Additionally, other cytokines we did not measure may be playing a role as well. We have yet to measure any lipid mediators to determine if the lipid production profile differs in the activated apoptotic neutrophils, but considering apoptotic cells are known to produce anti-inflammatory $\mathrm{PGE}_{2}$, it would be interesting to see if activated apoptotic neutrophils have a much different lipid production profile compared to apoptotic neutrophils. Additionally, activated apoptotic neutrophils were efferocytosed at the same rate as unstimulated neutrophils, showing the rate of efferocytosis was not contributing to this increased function of the activated apoptotic neutrophils. This set of data showcases the importance of 
how a further understanding of the mechanisms apoptotic cells are needed to be able to elicit a down-regulation of pro-inflammatory mediators and promote inflammation resolution. A further understanding of this process will aid in our ability to utilize these anti-inflammatory pathways as therapeutic targets. 


\section{Chapter 6}

\section{The Role of Prostaglandin E2 during Lyme Arthritis Resolution}

\section{Introduction}

Prostaglandins are cyclooxygenase (COX) derived eicosanoids that play various signaling roles throughout the inflammatory response. The prostaglandin $\mathrm{PGE}_{2}$ is metabolized from arachidonic acid by COX-1 or COX-2 to $\mathrm{PGH}_{2}$, which is then processed by PGE synthase-1 (mPGES-1) to $\mathrm{PGE}_{2}$ [155]. While $\mathrm{PGE}_{2}$ is traditionally thought of as pro-inflammatory, as it is thought to be a primary driver of rheumatoid arthritis pathology, recent literature suggests it can also be antiinflammatory and promote resolution [156]. Dying cells release $P \mathrm{PE}_{2}$ to function as an inhibitory DAMP and aid in downregulating the pro-inflammatory response [151]. The pro-inflammatory or pro-resolution function of $\mathrm{PGE}_{2}$ depends on which of the four E-prostinoid (EP) receptors it signals through. Activation through the EP1 or EP3 receptor increases intracellular calcium and decreases intracellular cAMP, respectively, which contributes to inflammation [157]. Signaling through EP2 or EP4 results in increased intracellular cAMP to inhibit the formation of the pro-inflammatory Leukotrienes and also to promote the production of the 12/15 lipoxygenase (12/15-LO) product lipoxin $A_{4}$ [158]. Non-steroidal anti-inflammatory drugs, such as ibuprofen, are COX inhibitors, and these prevent the production of $\mathrm{PGE}_{2}$. While the use of these therapies might alleviate the symptoms of arthritis associated with pro-inflammatory $\mathrm{PGE}_{2}$ signaling, including pain and swelling, they may prolong the inflammatory response. 
Our lab has previously used a lipidomics approach to arthritis-susceptible $\mathrm{C} 3 \mathrm{H}$ mice exhibit an increase of $\mathrm{PGE}_{2}$ production within the joints during times of arthritis induction, 7 to 14 days pi, and also during arthritis resolution, 28 days pi [108]. This dual spike in $\mathrm{PGE}_{2}$ production points to a role for $\mathrm{PGE}_{2}$ in both arthritis induction and resolution. Additional studies from our lab using COX-2-1mice have shown that in the absence of prostaglandin production, mice fail to resolve their arthritis, characterized by increased cellular infiltrate, as determined by histologic analysis, during and after resolution [104]. This may be due to the role of $\mathrm{PGE}_{2}$ in switching eicosanoid production from pro-inflammatory 5-LO, to pro-resolving 12/15-LO products. However, the use of Cox-2-- mice not only prevented $\mathrm{PGE}_{2}$ production, but the production of other prostaglandins as well. For these studies, we wanted to determine the specific role of $\mathrm{PGE}_{2}$ during Lyme arthritis resolution and determine its role during efferocytosis.

\section{Results}

\section{Macrophages produce $\mathrm{PGE}_{2}$ in response to $A C$ and $A C$ produce $P \mathrm{PE}_{2}$ in} vitro

Previously, we have shown the administration of $A C$ to $B$. burgdorferi-activated macrophages altered macrophage cytokine production to a more antiinflammatory state (Figure 5), therefore, we first investigated how the 

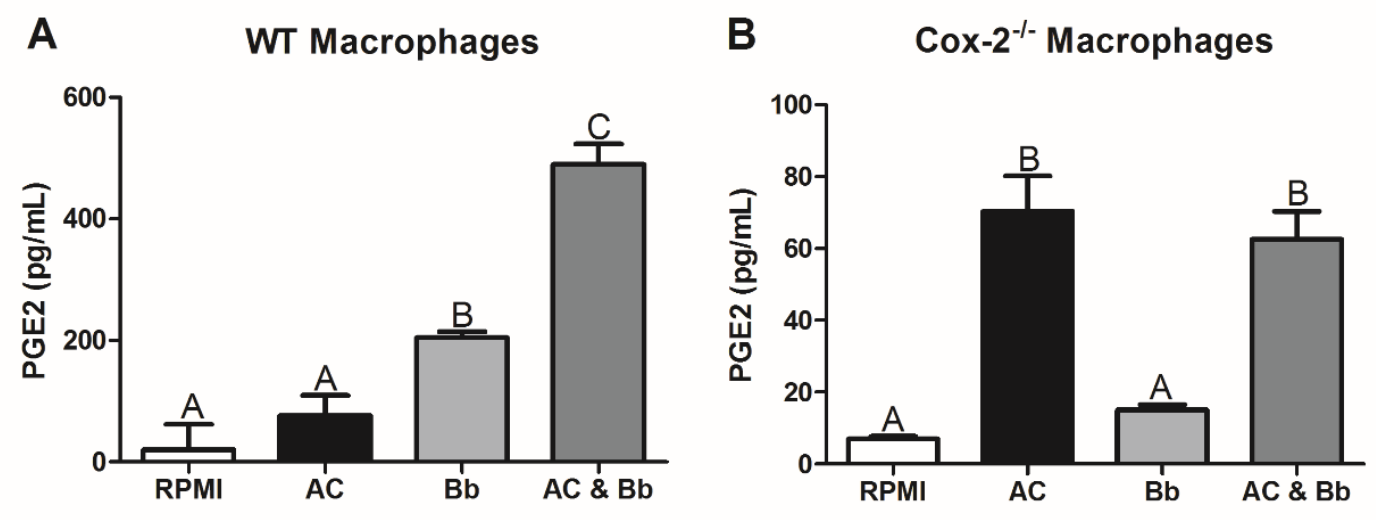

Figure 28: In vitro quantification of $\mathrm{PGE}_{2}$ in WT and Cox-2/- BMDM supernatant co-cultured with apoptotic Jurkat cells.

C3H WT (A) or C3H Cox-2-- (B) were co-cultured with apoptotic Jurkat cells and B. burgdorferi for 24 hours, and $\mathrm{PGE}_{2}$ was quantified by EIA. $n=3$ per group. Bars with different letters are significantly different from each other at the $P<0.05$ level. 
administration of $\mathrm{AC}$ altered macrophage production of $\mathrm{PGE}_{2}$. As shown in Figure $28 \mathrm{~A}$, the macrophages receiving both $B$. burgdorferi and $A C$ had significantly increased levels of $\mathrm{PGE}_{2}$ production compared to macrophages coculture with B. burgdorferi alone. Additionally, Cox- $2^{-/-}$macrophages, which are deficient in their ability to produce $\mathrm{PGE}_{2}$, still have levels of $\mathrm{PGE}_{2}$ when given $\mathrm{AC}$ (Figure 28B), confirming the results of others that $A C$ release $P \mathrm{FE}_{2}$ [151]. These data show macrophages make increased amounts of $P G_{2}$ in the presence of $\mathrm{AC}$, and that $\mathrm{AC}$ themselves can also produce $\mathrm{PGE}_{2}$.

\section{mPGES-1/- mice have decreased swelling and increased cellular infiltrate during inflammation resolution}

The apoptosis of both neutrophils and macrophages, and the efferocytosis of these cells is an important mechanism mediating Lyme arthritis resolution. Since $\mathrm{PGE}_{2}$ appears to have an anti-inflammatory role in this model, we next determined how inflammation resolution is impacted within the ankle joints during B. burgdorferi infection in the absence of $\mathrm{PGE}_{2}$. By using mPGES $-1 /$ mice, that are unable to produce $\mathrm{PGE}_{2}$, we found $\mathrm{mPGES}-1 \%$ mice had significantly decreased ankle swelling throughout infection (Figure 29). This shows edema reduction in these mice, however, ankle swelling does not always correlate with the underlying inflammation.

To further our understanding of the role of $\mathrm{PGE}_{2}$ during inflammation resolution, we determined how neutrophil and macrophage recruitment to the joints, and 


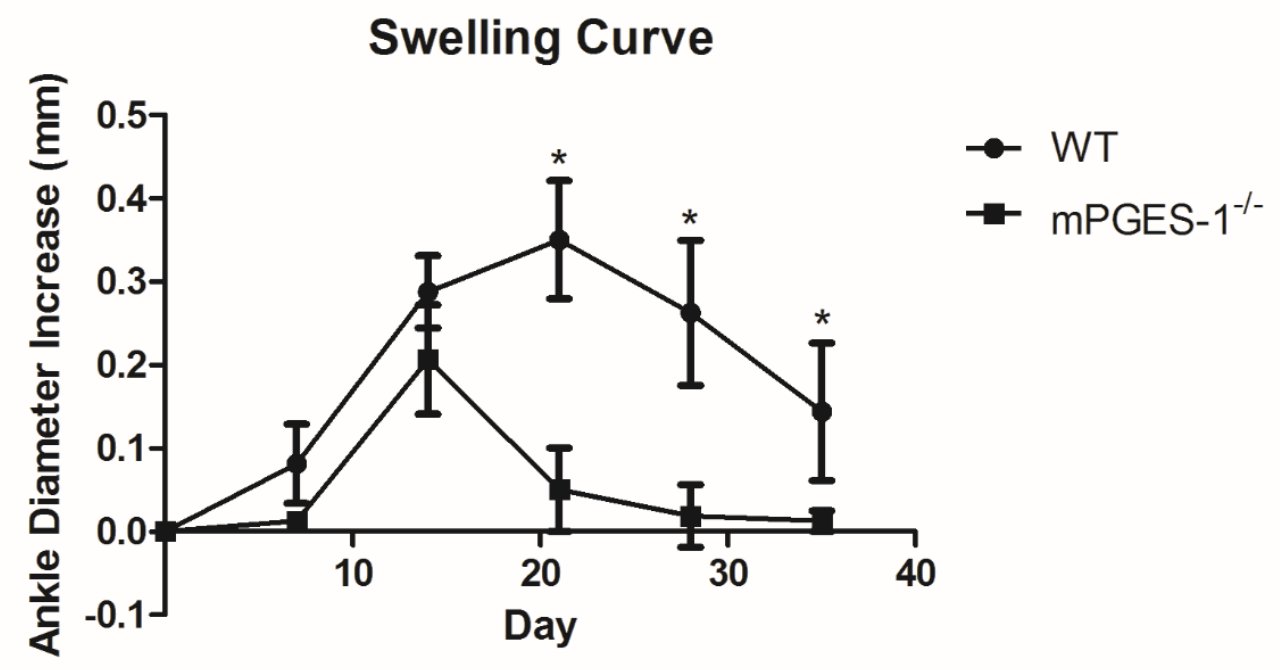

Figure 29: Ankle swelling in B. burgdorferi-infected WT and mPGES-1 ${ }^{-/-}$ mice.

C3H WT and mPGES-1/- mice were infected with $1 \times 10^{5}$ B. burgdorferi, and ankle swelling was monitored weekly 
how the apoptosis of these cells was impacted in the absence of PGE2.

Using flow cytometry, neutrophil and macrophage numbers and the percentage of apoptotic neutrophils and macrophages were evaluated in B. burgdorferiinfected mice during inflammation resolution. As shown in Figure 30, the number of neutrophils in the ankle joints was reduced at day 21 pi (Figure 30A) in the mPGES $-1^{-/-}$mice compared to WT, however neutrophil numbers increased by day 28 pi (Figure 30B), and the number of neutrophils returned to WT levels on day 35 pi (Figure 30C). Additionally, there was no different in the percentage of apoptotic neutrophils between mPGES-1/- and WT mice (Figure 30D-F) at any timepoints, suggesting the number of live neutrophils was always in a similar ratio to apoptotic neutrophils in both strains.

While the number of macrophages was significantly lower in the mPGES-1\% mice at day $21 \mathrm{pi}($ Figure 31A) with the same percentage of apoptotic macrophages (Figure 31D), there was a significant influx in the number of macrophages in the mPGES-1\% mice compared to WT at both 28 (Figure 31B) and 35 (Figure 31C) days pi. This influx in macrophage number was also accompanied by a significant reduction in the percentage of apoptotic macrophages at days 28 (Figure $31 \mathrm{E})$ and 35 (Figure 31F) pi. The data suggests while ankle swelling is reduced in the mPGES $-1 \%$ mice, the mice have increased cellular infiltrate, primarily non-apoptotic macrophages, during the resolution of inflammation, resulting in a prolonged inflammatory response. This increase in macrophages with fewer apoptotic macrophages could be contributing to the inflammatory 
A
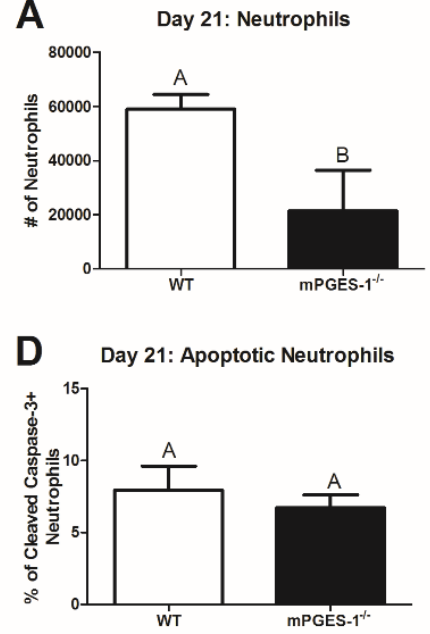

B

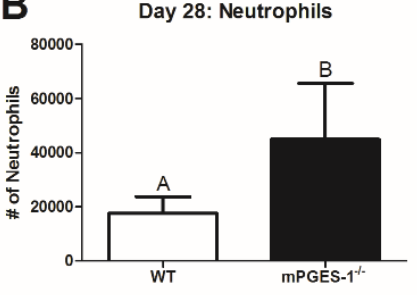

E

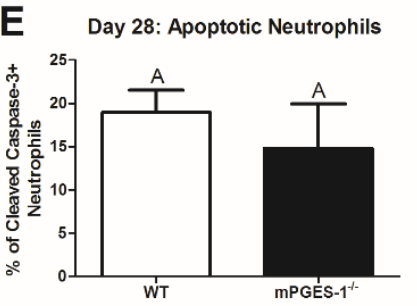

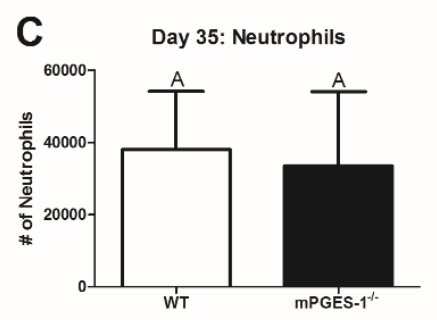

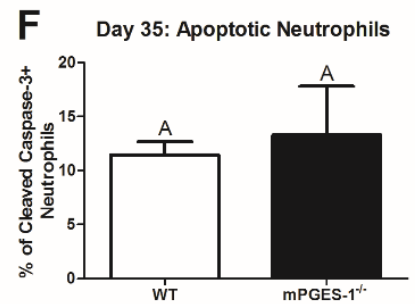

Figure 30: Quantification of neutrophils and apoptotic neutrophils within B. burgdorferi-infected ankle joints in C3H WT and mPGES-1/-- mice.

Mice were infected with $1 \times 10^{5}$ B. burgdorferi and sacrificed at indicated days for flowy cytometry analysis of joint inflammatory cells. Total number of neutrophils $(A-C)$ and the percentages of cleaved-caspase $3^{+}$neutrophils (D-F) of total CD45.2+ inflammatory cells were evaluated on 21,28 , and 35 days pi. $n=4$ per group. Bars with different letters are significantly different from others at the $p<0.05$ level. 

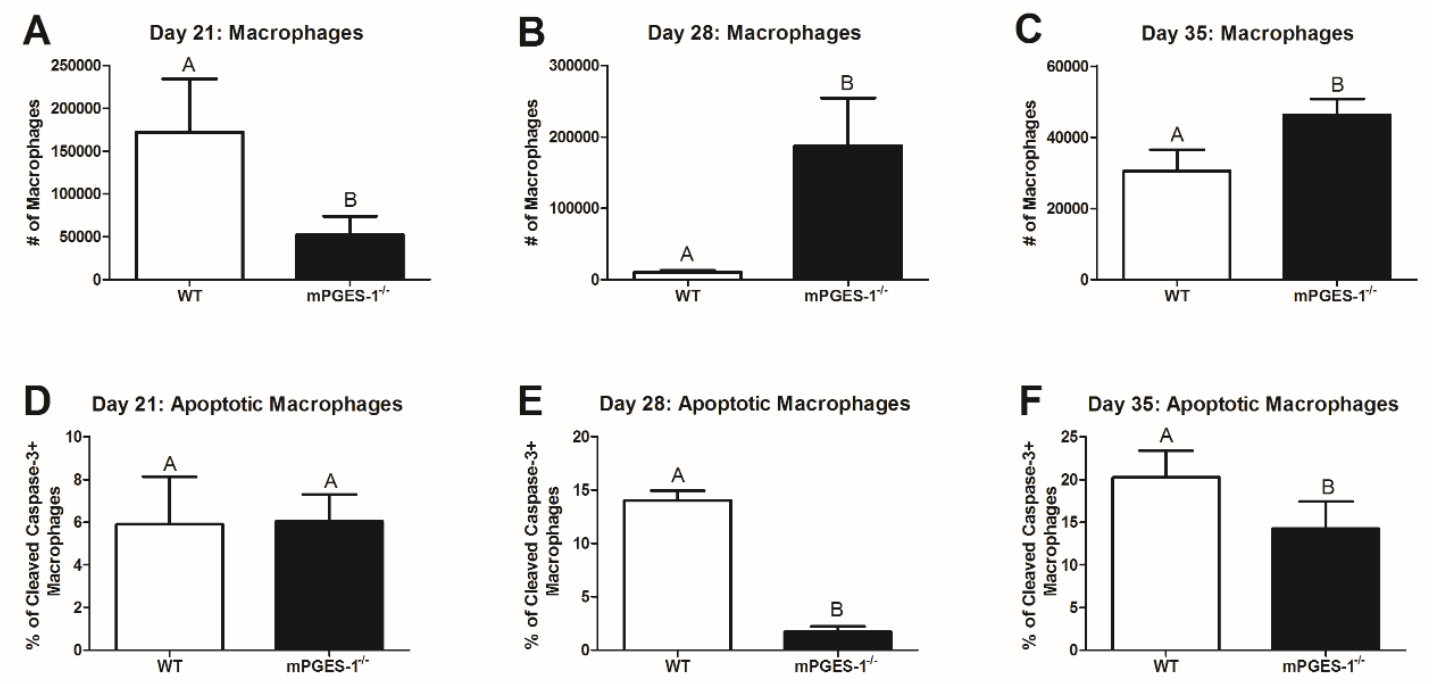

Figure 31: Quantification of macrophages and apoptotic macrophages within B. burgdorferi-infected ankle joints in C3H WT and mPGES-1/- mice.

Mice were infected with $1 \times 10^{5} \mathrm{~B}$. burgdorferi and sacrificed at indicated days for flowy cytometry analysis of joint inflammatory cells. Total number of macrophages (A-C) and the percentages of cleaved-caspase $3^{+}$macrophages (D-F) of total CD45.2+ inflammatory cells were evaluated on 21,28 , and 35 days pi. $n=4$ per group. Bars with different letters are significantly different from others at the $p<0.05$ level. 
response and delaying inflammation resolution in the absence of $\mathrm{PGE}_{2}$. This same increase of cellular infiltrate was shown in the COX-2-/- mice [104], suggesting the reason for the inability for those mice to undergo inflammation resolution may be due to lack of $\mathrm{PGE}_{2}$ signaling.

\section{mPGES-1//- macrophages have altered responses to apoptotic neutrophils in vitro}

To investigate the impact of the increased number of macrophages in the mPGES $-1{ }^{-/}$mice during $B$. burgdorferi infection, we determined if $\mathrm{PGE}_{2}$ plays a role in macrophage cytokine production in the presence of apoptotic neutrophils. We have previously shown apoptotic neutrophils can alter macrophage cytokine production when in the presence of $B$. burgdorferi (Figure 6), and this change in macrophage phenotype can aid in the down-regulation of inflammatory responses. As shown in Figure 32A-C, both WT and mPGES-1/- apoptotic neutrophils can cause a similar alteration of macrophage cytokine production in WT macrophages. However, both WT and MPGES $-1^{-/-}$apoptotic neutrophils were unable to alter cytokine production for TNF- $\alpha$ (Figure 32D) KC (Figure 32E), or IL-10 (Figure 32F). This suggests that apoptotic cells are only able to induce an alteration in cytokine production if the macrophage can make $\mathrm{PGE}_{2}$. While apoptotic cells can make $\mathrm{PGE}_{2}$ (Figure 28B), macrophage production of $\mathrm{PGE}_{2}$ is needed to decrease pro-inflammatory and increase anti-inflammatory cytokine production in response to apoptotic neutrophils. 

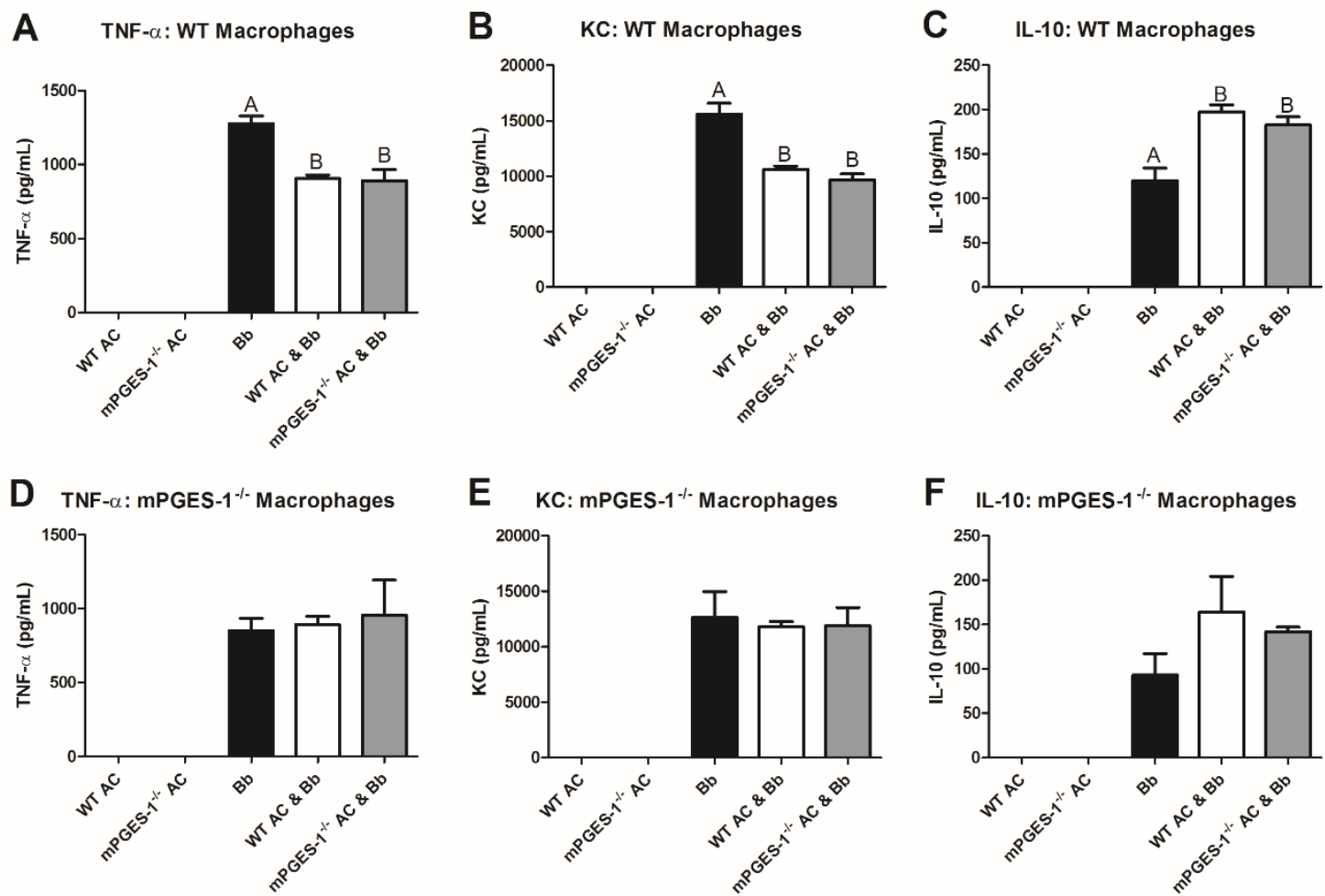

Figure 32: In vitro quantification of cytokines in C3H WT and mPGES-1/BMDM supernatant co-cultured with WT or MPGES-1//- apoptotic neutrophils.

C3H WT (A-C) and mPGES-1- (D-F) BMDM were co-cultured with either WT or mPGES-1 ${ }^{-1}$ apoptotic neutrophils and $B$. burgdorferi for 24 hours, and cytokine levels of TNF- $\alpha(A, D), K C(B, E)$, and IL-10 (C,F) were quantified by ELISA. $n=3$ per group. Bars with different letters are significantly different from each other at the $p<0.05$ level. 
The efferocytosis of apoptotic neutrophils by phagocytic cells aids in the downregulation of inflammation and increases PPAR- $p$ transcription during $B$. burgdorferi infection. Therefore, we wanted to determine if efferocytosis of apoptotic neutrophils or PPAR- $\gamma$ increase by apoptotic neutrophils would change without PGE2. There was no difference in efferocytosis in WT macrophages engulfing either WT or mPGES-1\% apoptotic neutrophils (Figure 33), however mPGES-1/- macrophages had significantly decreased efferocytosis of both WT and mPGES-1/- apoptotic neutrophils. This suggests that macrophages unable to make $\mathrm{PGE}_{2}$ have a defect in the efferocytosis of apoptotic neutrophils.

PPAR- $\gamma$ transcription is upregulated in the presence of apoptotic neutrophils and plays an important role during inflammation resolution. As shown in Figure 34, transcription of PPAR- $\uparrow$ was significantly increased in B. burgdorferi co-cultured macrophages also given either WT or mPGES-1/- apoptotic neutrophils. mPGES-1\% macrophages had similar levels of PPAR- $\gamma$ transcripts in both $B$. burgdorferi co-cultured macrophages and also those given either WT or MPGES$1^{-/}$apoptotic neutrophils. The mPGES-1/- macrophages did not have enhanced PPAR- $\gamma$ transcription with the administration of apoptotic neutrophils, unlike WT macrophages. These data provide further evidence that macrophages need autocrine $\mathrm{PGE}_{2}$ signaling for apoptotic neutrophils to stimulate inflammation resolution. 


\section{Macrophage Efferocytosis}

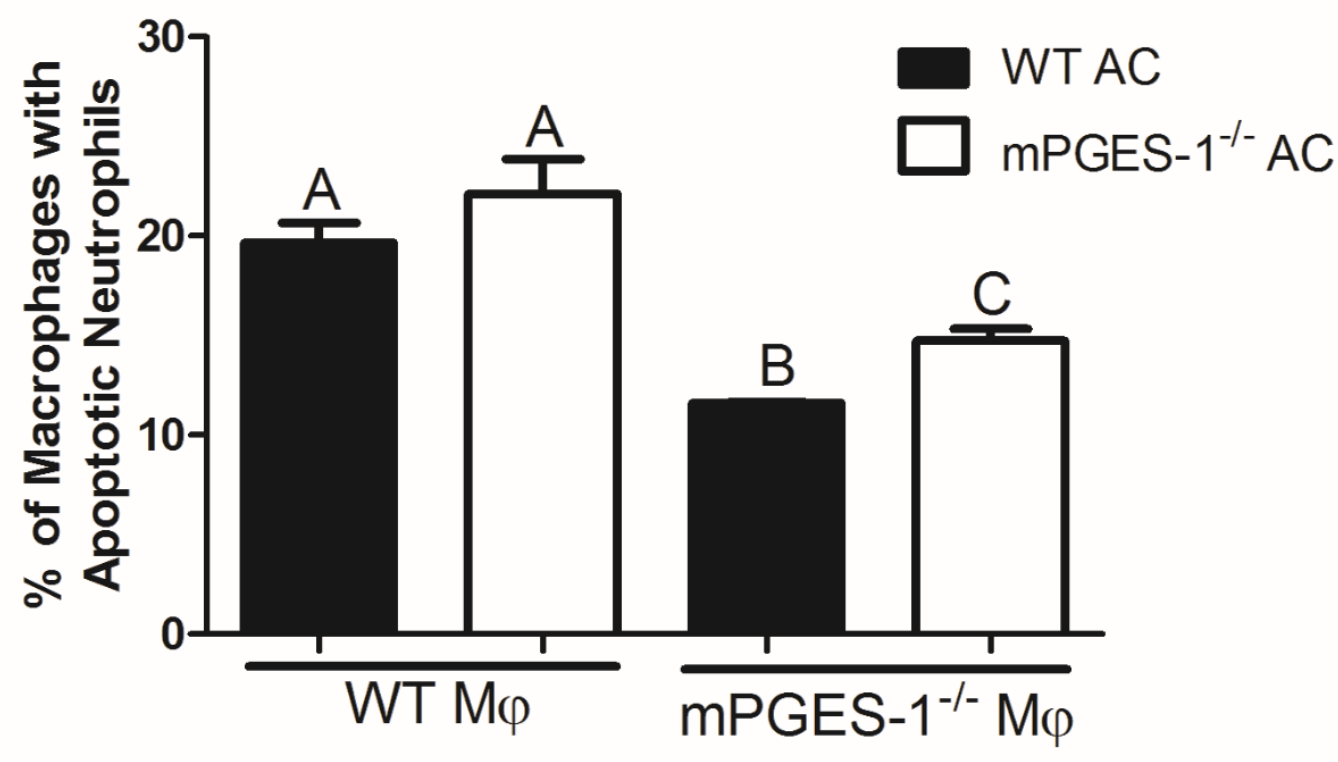

Figure 33: C3H WT and mPGES-1/- BMDM efferocytosis of WT and mPGES$1^{-/-}$apoptotic neutrophils.

C3H WT or mPGES-1/-BMDM were given either CFSE-labeled WT or mPGES$1^{-/}$apoptotic neutrophils at a 2:1 concentration for 4 hours. The percentage of macrophages containing apoptotic neutrophils was determined by flow cytometry. $n=3$ per group. Bars with different letters are significantly different from each other at the $p<0.05$ level. 


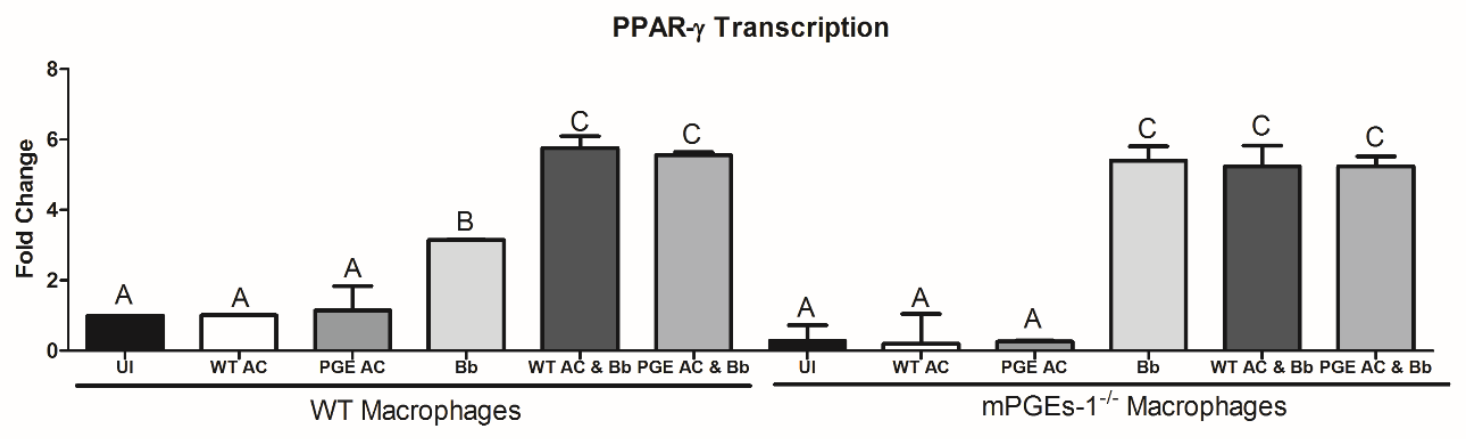

Figure 34: C3H WT and mPGES-1/- apoptotic neutrophil induction of PPAR$Y$ in WT and $\mathrm{mPGES}-1^{-/-}$BMDM.

C3H WT and mPGES-1/- were co-cultured with either WT or mPGES-1/apoptotic neutrophils and/or B. burgdorferi for 24 hours. PPAR-y transcription was determined by real-time PCR. $n=3$ per group. Bars with different letters are significantly different from each other at the $p<0.05$ level. 


\section{Discussion}

While we have shown apoptotic cell clearance is important during inflammation resolution in Lyme arthritis, we are still trying to determine the mechanisms involved and the mediators involved. Eicosanoid pathways are prevalent in the regulation of inflammatory responses, therefore we wanted to determine the role of $\mathrm{PGE}_{2}$, an important lipid mediator in other inflammatory models, during Lyme arthritis. We show here that while apoptotic cells are able to produce PGE2 as previously described, $\mathrm{PGE}_{2}$ production by macrophages is essential in their response to apoptotic neutrophils. mPGES-1/- mice had decreased swelling throughout infection compared to WT mice, however, mPGES-1/- mice had increased inflammatory infiltrate during inflammation resolution. Because of the dual signaling role of $\mathrm{PGE}_{2}$, this may explain why decreased swelling was noted in the mPGES-1\% mice, due to the fact the pro-inflammatory form of $\mathrm{PGE}_{2}$ signaling through EP1 or EP3 did not occur, but the consequences of the inability to signal anti-inflammatory $\mathrm{PGE}_{2}$ through EP2 or EP4 also affected these mice. Additionally, pro-inflammatory $\mathrm{PGE}_{2}$ production may also be important for resolution, as inhibition of COX-2 in $B$. burgdorferi-infected mice early in infection also resulted in exacerbated cellular infiltrate during resolution [104].

The increased cellular infiltrate within the joints of mPGES-1/- mice was from macrophages, rather than neutrophils as we have shown in $\mathrm{BLT1} 1^{-/}$mice. Additionally, a significantly lower percentage of these macrophages were 
apoptotic, emphasizing an imbalance in the ratio of live cells to apoptotic cells. This suggests the macrophages may have increased recruitment and are living longer in B. burgdorferi-infected mPGES-1/- mice compared to WT during the resolution stages. Because macrophages seem to be the cell out of balance during resolution in the absence of $\mathrm{PGE}_{2}$, we wanted to then characterize macrophage function in respects to their interaction with apoptotic neutrophils. We show here that while WT and mPGES-1/- apoptotic neutrophils are able to elicit similar changes in cytokine production in WT macrophages, as previously shown, mPGES-1/- macrophages are not affected by either WT or mPGES-1/apoptotic neutrophils. Additionally, mPGES $-1 /$ mice have a defect in apoptotic neutrophil efferocytosis, and apoptotic neutrophils do not increase PPAR- $\nu$ production in macrophages. This shows apoptotic neutrophils are unable to elicit the downregulation of the immune response through pro-inflammatory cytokine repression and anti-inflammatory cytokine production in mPGES-1 ${ }^{-1}$ macrophages. Further, efferocytosis, a major mechanism during inflammation resolution is defective in mPGES-1/- macrophages, suggesting $\mathrm{PGE}_{2}$ production by macrophages is important in the downregulation of immune responses during an inflammation. Overall, these data show the importance of $\mathrm{PGE}_{2}$ during inflammation resolution and suggest macrophage, rather than neutrophil production of $\mathrm{PGE}_{2}$ is more important in the anti-inflammatory response. 


\section{Chapter 7}

\section{Discussion}

Experimental Lyme arthritis in mice is caused by infection with the spirochete $B$. burgdorferi and causes chronic inflammation of the weight bearing joints. While much is known how inflammation is initiated through the over-recruitment of innate immune cells, there is little evidence for how resolution of inflammation is initiated in this disease. Because mice are able to resolve their arthritis during $B$. burgdorferi infection, unlike many other murine models of arthritis, such as the CIA model of rheumatoid arthritis, this provides an excellent model to determine the mechanisms of inflammation resolution.

\section{Apoptotic cell detection and administration}

While the clearance of $\mathrm{AC}$, especially apoptotic neutrophils, was hypothesized to initiate inflammation resolution during Lyme arthritis, there was little evidence for the occurrence of this phenomenon in vivo [76]. We show the increase in both number and percentage of apoptotic neutrophils and macrophages correlates with decreased ankle swelling and inflammation resolution during Lyme arthritis (Figure 3). Additionally, we used cleaved-caspase $3^{+}$as a marker for apoptosis. Previously, it was common to use Annexin V, which stains phosphatidylserine, in conjunction with a viability dye, such as $7-A A D$, to determine whether cells were live, in early apoptosis, in late apoptosis, or dead [159]. This issue with this method of $A C$ detection is there is no way to determine the difference between 
cells in late apoptosis (Annexin $\left.\mathrm{V}^{+}, 7-\mathrm{AAD}^{+}\right)$and pyroptotic or necrotic cells, as the Annexin $\mathrm{V}$ cannot distinguish between phosphatidylserine on the cell surface as with $A C$ vs inside the cell, as with all other cell types [159]. This causes difficulty when trying to characterize the form of death cells are undergoing during an inflammatory response. In addition, when using Annexin $\mathrm{V}$, it can be difficult or impossible to stain for additional cellular markers, such as F4/80 for macrophages or Ly6G for neutrophils, to determine which cell type is apoptotic due to the binding requirements of Annexin $\mathrm{V}$ antibody. The use of Annexin $\mathrm{V}$ is more applicable in vitro in homogeneous cell populations, but using cleavedcaspase 3 to determine apoptosis in vivo is the only way specific AC populations can be identified. Additionally, due to the fact cleaved-caspase 3 is an executioner caspase, it does not play a role in any other forms of cell death besides apoptosis, meaning it can better identify AC without also potentially staining pyroptotic or necrotic cells [133]. By defining the roles of apoptosis of neutrophils and macrophages and the efferocytosis of these cells during inflammation resolution, it will give better insight to how inflammation can be downregulated in chronic inflammatory diseases.

Mechanisms of inflammation resolution with $A C$ shown previously have been in autoimmune models, including lung fibrosis and rheumatoid arthritis [5, 77]. We show here AC play a role in inflammation resolution during Lyme arthritis, and similar to other chronic inflammatory models, are able to promote earlier arthritis resolution following their administration (Figure 4). Previously, others have shown 
the treatment of mice with large quantities of $A C\left(10^{7}-10^{8}\right)$ administered intraperitoneally (i.p.) or intravenously (i.v.) elicited a strong anti-inflammatory response in autoimmune arthritis models $[77,160,161]$. While this treatment strategy was able to downregulate inflammation, a large number of apoptotic cells was required for successful treatment. In our studies, we administered the AC locally in an attempt to reduce the number of $A C$ needed to elicit a response. By using a more localized treatment, we were able to reduce the number of $A C$ needed to elicit a response. Additionally, we were administering AC after arthritis induction as a therapeutic, rather than a preventative, which is more applicable to human Lyme arthritis treatment.

Various other inflammatory models have given AC both therapeutically and prophylactically $[5,77]$. However, during infectious diseases such as Lyme arthritis, some inflammation is needed for pathogen recognition and clearance by the immune system and differs from autoimmune models in which inflammation is not needed. We had to alter the time of $A C$ administration compared to previously established models to avoid downregulating immune responses before bacterial clearance occurred. This is the first infectious disease model in which the administration of $A C$ was able to initiate earlier inflammation resolution through the decreased recruitment of inflammatory cells. Additionally, the decreased recruitment of neutrophils by AC administration did not interfere with B. burgdorferi clearance, as both control and AC treated mice had similar bacterial loads throughout infection (Figure 4). Earlier administration of AC may 
have reduced the host response to $B$. burgdorferi, due to $A C$ downregulation of neutrophil and macrophage effector functions, including B. burgdorferi

phagocytosis (Figure 7). Additionally, the similarity in B. burgdorferi loads while having differences in ankle swelling in the control mice compared to $A C$ treated also demonstrates the point that the presence of $B$. burgdorferi does not drive the arthritis, but rather the over-recruitment of the innate immune cells in response to B. burgdorferi drives arthritis severity. This was supported previously when mice with exacerbated arthritis and ankle swelling had normal B. burgdorferi antibody production and pathogen clearance $[3,104]$.

\section{Localized administration of apoptotic cell-associated therapeutics}

While we were able to show AC treatment of macrophages resulted in significant alterations in cytokine production in vitro (Figure $5 \& 6$ ), we could not demonstrate this in vivo (Figure 4). This may be partially due to the limited number of $A C$ that could be injected as a localized treatment, as only around 10 $\mu \mathrm{l}$ can be injected into the tibio-tarsal synovial cavity. While increasing the number of $A C$ per dose or increasing the number of doses may have had a much stronger effect in downregulating inflammation, we are trying to develop a cellfree mechanism to promote arthritis resolution, rather than increasing $A C$ treatment frequency. By promoting the similar effects as $\mathrm{AC}$ on inflammation resolution without the $\mathrm{AC}$ themselves would be a more appropriate treatment in humans for therapy of non-resolving inflammatory diseases. 
The transcription factor PPAR- $\gamma$ has been previously shown in other inflammatory models to be upregulated during $A C$ administration [5], however, we show here administration of the PPAR- $\gamma$ agonist rosiglitazone was able to elicit similar effects as the apoptotic cell treatment (Figure 11). Additionally, we show that like $\mathrm{AC}$, rosiglitazone is able to decrease ankle swelling and decrease neutrophil recruitment to the joints. This is the first localized therapeutic that mimics the administration of $\mathrm{AC}$ in an inflammatory infectious disease, showcasing the potential for utilizing the apoptotic pathway mechanisms for human therapeutics. Because PPAR- $\gamma$ performs several functions within the cell besides regulation of inflammation, including fatty acid storage and glucose metabolism, systemic administration is difficult without disrupting other cellular processes and functions [162]. Rosiglitazone was originally developed as a type 2 diabetes medication for

its ability to regulate glucose metabolism, however, it also caused an increase in coronary artery disease prevalence during long term oral administration [163]. Instead of a systemic treatment with PPAR- - , we tried to utilize a localized treatment, taking advantage of the fact the arthritis is localized to the ankle and knee joints and also to avoid long term complications. This local administration method of rosiglitazone is also more similar to our AC treatment regimen than in other models $[5,77]$.

\section{Ankle swelling and cellular infiltrate are not always correlated}

Ankle swelling is used to assess arthritis severity during Lyme arthritis, as well as other forms of arthritis, along with cellular infiltrates and levels of inflammation 
within the joint as determined by histology and flow cytometry. However, ankle swelling does not always correlate to the underlying inflammation. As shown with the BLT1 ${ }^{-/}$mice, ankle swelling was only increased on day 35 pi (Figure 12), but these mice had exacerbated neutrophil infiltrate throughout inflammation resolution (Figure 13). Additionally, the mPGES-1/- mice had decreased ankle swelling throughout $B$. burgdorferi infection compared to WT mice (Figure 29) however had increased macrophage infiltration during resolution compared to WT (Figure 31). These two examples demonstrate that while ankle swelling might indicate the size of the edema, it does not always correlate to the cellular infiltration and underlying inflammation within the joint, especially since $P G_{2}$ is a known regulator of edema [156].

\section{The role of $\mathrm{LTB}_{4}$ signaling and $\mathrm{PGE}_{2}$ production during inflammation resolution}

The mediators responsible for the induction of inflammation can vary among models and arthritis types. For example, the pro-inflammatory lipid $\mathrm{LTB}_{4}$ and its downstream signaling has been shown to play a role in the induction of rheumatoid arthritis. Mice deficient in 5-LO-activating protein (FLAP) had decreased disease onset in the collagen-induced arthritis model [124], and blocking BLT1 signaling with BLT1\% mice resulted in complete protection from CIA development [164]. A similar phenomenon occurred in the $\mathrm{K} / \mathrm{BxN}$ serum transfer model of rheumatoid arthritis, in which neutrophil recruitment through $\mathrm{LTB}_{4}$ signaling was required for disease [165]. 
However in contrast to the above studies, we show here blocking of BLT1 signaling through the use of $\mathrm{BLT1} \%$ mice resulted in exacerbated neutrophil infiltrate during resolution and caused prolonged inflammation (Figure $12 \& 13$ ). Although rheumatoid arthritis and Lyme arthritis are both chronic inflammatory diseases, they have different roles for the signaling of LTB 4 through BLT1. Even though many of the mechanisms of disease pathogenesis may be similar, Lyme arthritis is an infectious arthritis and rheumatoid arthritis models try to recapitulate the onset of an autoimmune disease. Therefore, therapies need to be verified in various inflammatory models due to the potential for different roles of various mediators, because blocking of $\mathrm{LTB}_{4}$ signaling may aid in preventing rheumatoid arthritis development but causes exacerbated inflammation during Lyme arthritis and possibly other inflammatory diseases.

The blocking of inflammation and inflammatory lipid signaling is a commonly used strategy for developing arthritis therapeutics. Many individuals frequently use ibuprofen, a COX inhibitor, to alleviate the unpleasant symptoms of arthritis, such as pain and swelling. However, the administration of these drugs may delay resolution and in turn prolong inflammation, as previously shown in $\mathrm{COX}-2^{--}$mice infected with B. burgdorferi [104]. Additionally, some of the pro-inflammatory lipids also have anti-inflammatory signaling ability as well. PGE 2 is the main driver of inflammation during rheumatoid arthritis [166], however, it possibly has a both pro- and anti-inflammatory role during Lyme arthritis. $\mathrm{PGE}_{2}$ may be 
responsible for arthritis induction, but it also plays a key role during resolution. Lipidomics in $B$. burgdorferi-infected mice showed increased $\mathrm{PGE}_{2}$ both during arthritis onset and resolution [108]. Additionally, mPGES-1/- mice have decreased ankle swelling and cellular infiltrate early in $B$. burgdorferi infection, however, while their ankle swelling remains decreased (Figure 29), there are nearly four times as many infiltrating macrophages during the resolution stages (Figure 31). This increased infiltrate causes exacerbated arthritis within the joints and prolongs inflammation, even with decreased ankle swelling.

$\mathrm{LTB}_{4}$ is a pro-inflammatory eicosanoid responsible for the recruitment of neutrophils. Blocking of $\mathrm{LTB}_{4}$ has been shown to prevent disease onset and severity in rheumatoid arthritis [164], but it also plays a role during resolution in Lyme arthritis. Without $\mathrm{LTB}_{4}$ signaling, $\mathrm{BLT1}^{-/-}$neutrophils are unable to downregulate CAMP, which prevents DISC complex formation (Figure 19). This decrease in DISC formation is responsible for decreased apoptosis and efferocytosis of apoptotic neutrophils and prolongs inflammation. Additionally, neutrophils can be recruited by other mechanisms besides LTB4. Even though $\mathrm{BLT1}^{-/-}$neutrophils do not migrate to $\mathrm{LTB}_{4}$ as well as WT neutrophils, they can still migrate to other chemo-attractants, such as KC during Lyme arthritis [100]. This shows there can be multiple ways cellular recruitment during an inflammatory response can occur and blocking one path of neutrophil recruitment does not always mean the ability to recruit neutrophils is completely lost. Also, 
blocking the signaling of these pro-inflammatory mediators can have downstream consequences during the resolution phase as well.

Eicosanoids play an important role during both arthritis induction and resolution, however, certain eicosanoids are required by specific cell types to perform these roles. It is important for neutrophils to be able to signal $\mathrm{LTB}_{4}$ through the BLT1 receptor to be able to undergo efficient apoptosis and be efferocytosed by macrophages (Figure 15). Additionally, BLT1 ${ }^{-/}$macrophages were able to efferocytose WT apoptotic neutrophils at a similar rate as WT macrophages, showing BLT1 signaling on the macrophage is not critical for efferocytosis. However, both WT and BLT1/- macrophages were unable to efficiently efferocytose BLT1 ${ }^{-/}$apoptotic neutrophils (Figure 15D), showing BLT1 signaling in neutrophils is important for efferocytosis. This suggests a defect in neutrophil signaling is likely responsible for the defect in inflammation resolution found in $\mathrm{BLT1}{ }^{-/}$mice infected with $B$. burgdorferi.

One major finding of this work is that macrophage production of $\mathrm{PGE}_{2}$ is critical for their modulation by apoptotic neutrophils. Even though apoptotic neutrophils were able to make $\mathrm{PGE}_{2}$ [151] (Figure 28B), macrophage production of $\mathrm{PGE}_{2}$ was required to signal the downregulation of cytokines in response to apoptotic neutrophils. WT and mPGES-1/- apoptotic neutrophils were able to alter cytokine production of $B$. burgdorferi-activated WT macrophages, showing production of PGE2 by apoptotic neutrophils was not required, however, neither WT and 
mPGES- $1^{-/-}$apoptotic neutrophils were able to alter cytokine production in mPGES-1/- macrophages (Figure 32). Additionally, WT macrophages could efferocytose WT and mPGES-1/- apoptotic neutrophils at the same rate, but mPGES-1\% macrophages were defective in efferocytosing either WT or mPGES$1^{-/}$apoptotic neutrophils (Figure 33). This demonstrates $\mathrm{LTB}_{4}$ and $\mathrm{PGE}_{2}$ act at different points in the apoptotic pathway to coordinate clearance of apoptotic cells, where BLT1 signaling is important for neutrophil apoptosis and macrophages require endogenous $\mathrm{PGE}_{2}$ to perform effector functions associated with apoptotic neutrophils. This defect in PGE2 production may also explain the exacerbated number of macrophages shown during inflammation resolution in mPGES-1/- mice (Figure 31).

Many eicosanoid pathway knockout mice have only been available for a limited time, so previously, others have utilized various inhibitors during in vitro cultures to determine the role of the eicosanoids [167]. While this gives a general idea of the role of the mediators being inhibited, it does not separate the role of the mediators in different cell types. As shown here, BLT1 signaling is needed for neutrophils, while $\mathrm{PGE}_{2}$ production is important for macrophages during apoptosis and efferocytosis. If inhibitors were used to determine these functions in vitro, it would have been impossible to determine which cell type was responsible for the defect, and it would be difficult to come to a mechanistic conclusion to explain the exacerbated cellular infiltrate and arthritis in both cases. The ability to tease apart differences in eicosanoid signaling requirements for the 
different immune cell types will allow for a better understanding of the roles of the eicosanoids during inflammation resolution.

The use of flow cytometry to identify and quantify cellular infiltrates within inflamed mouse joints is a relatively recent development $[98,112]$. Prior to this, studies were done using only histological analysis of the H\&E stained slides with severity scores provided by a blinded observer $[2,3]$. When using flow cytometry to determine cellular populations of inflammatory cells within tissues, it is important to look at data in several different ways to fully understand what is occurring in vivo. We were able to determine the number of neutrophils and macrophages throughout $B$. burgdorferi infection within the ankle joints of mice. Using additional cellular markers, determining phenotypic differences between these cells was also possible. By using cleaved-caspase 3 antibody, we were able to determine the number of apoptotic neutrophils and macrophages within the inflamed joint. In addition, the percentage of these apoptotic cells was also determined, which gave a different perspective on the data. While $\mathrm{BLT1} 1^{--}$joints contained a similar number of apoptotic neutrophils as WT, they had a significantly decreased percentage of apoptotic cells due to the increase in the number of live neutrophils (Figure 13). If only the number of apoptotic cells had been assessed, we would have detected no difference between BLT1 ${ }^{-/}$and WT mice, however, when considering the total number of neutrophils provides additional information. Often the inflammatory response is based on the signals present and the quantity of these signals. Therefore the ratio of live to apoptotic 
neutrophils and macrophages should be considered when assessing the mechanisms of inflammation resolution and the defects that can arise in knockout mice.

$\mathrm{LTB}_{4}$ signals through two receptors, BLT1 and BLT2. BLT1 is the high affinity receptor, while $B L T 2$ is the low affinity receptor, and $L_{T} B_{4}$ preferentially signals through BLT1 if both receptors are present [135]. For certain mechanisms, BLT2 can substitute for the absence of BLT1, such as in macrophage phagocytosis of B. burgdorferi [134]. However, high concentrations of LTB4 were required for BLT2 to function similar to BLT1. We noted a similar phenomenon in which BLT1I- neutrophils were unable to migrate to $\mathrm{LTB}_{4}$, even though they have a functional $B L T 2$ receptor (Figure $15 C$ ). This shows while $\mathrm{LTB}_{4}$ can signal through $\mathrm{BLT2}$, it requires a higher concentration of $\mathrm{LTB}_{4}$. This shows while there might be multiple receptors for a signaling mediator, it does not always mean they signal and function in the same capacity under the same conditions as other receptors.

Eicosanoids are produced through enzymatic reactions, and often the removal of an enzyme affects the synthesis of several mediators, rather than just one. For example, rofecoxib, marketed as Vioxx as an anti-inflammatory NSAID for osteoarthritis and acute pain conditions, inhibited COX-2. The drug was taken off the market five years later due to increased risks of heart attack and stroke associated with long-term usage [168]. The main mechanism proposed to explain these side effects was the suppression of prostacyclin, an anti-clotting agent in 
the blood [169]. Because Vioxx specifically inhibited the COX-2 enzyme, prostacyclin production decreased in endothelial cells and led to problems with declumping and vasodilation [169]. In addition to these concerns, others have hypothesized cardiotoxicity may be associated with anhydride metabolites formed when Vioxx became ionized during physiological conditions [168]. This shows by blocking an enzyme like COX-2, that is responsible for the production of several lipid mediators, unintended side effects can result from trying to block inflammation. A similar phenomenon was previously shown during Lyme arthritis in the inhibition of both COX-2 and 5-LO enzymes [3, 104]. While inhibition of these enzyme was intended to prevent inflammation, it ultimately resulted in exacerbated and prolonged arthritis.

Another important point to consider when working within enzymatic systems is substrate bioavailability. If an enzyme is removed from the system, its substrate may now be available for use by other enzymes and result in increased production of other mediators due. For example in mPGES-1/ mice, other prostaglandins, including thromboxane $\mathrm{B}_{2}, \mathrm{PGD}_{2}, \mathrm{PGF}_{2 \alpha}$ and 6-keto-PGF $\mathrm{P}_{1 \alpha}$, were reported to be significantly elevated in stomach tissue during LPS-stimulation [170]. This demonstrates that even if a phenotype is seen in a knockout mouse, it does not necessarily mean the lack of that specific enzymatic product or signaling causes the phenotype. The increased production of other metabolites could also cause the change in phenotype. This could explain the alteration in phenotype seen in mPGES-1/- macrophages in response to the apoptotic 
neutrophils. These macrophages could be making increased amounts of other eicosanoids resulting in the inability of apoptotic neutrophils to alter their production of cytokines (Figure 32) and the defect in macrophages to detect apoptotic neutrophils for efferocytosis as well (Figure 33).

Arachidonic acid is released from membrane stores by the action of phospholipase $A_{2}$, which is readily available in the cytosol of cells. While most cells have the potential to produce the various derivatives of arachidonic acid, it does not mean their signaling receptors are expressed on every cell type in the same abundance as well. Both BLT1 and BLT2 signal in response to LTB4, however, their expression on cell types and ligand specificity is vastly different. BLT1 is mainly found only on leukocyte subsets, while BLT2 is more constitutively expressed on cells throughout the body [131]. Some cells may produce greater amounts of certain eicosanoids than others. Both neutrophils [151] and macrophages [171] are able to produce PGE2, however, macrophage production of $\mathrm{PGE}_{2}$ is much greater than that of neutrophils. These differences in expression of receptors and production of eicosanoids can explain why certain mediators are required by specific immune cells during the inflammatory response and also during resolution.

\section{Secreted factors of apoptotic cells}

The process of acquiring $\mathrm{AC}$ for both in vitro and in vivo is labor intensive. Inducing apoptosis in Jurkat cells or harvesting neutrophils and inducing 
apoptosis requires planning to time everything correctly. Because of this, other groups have resorted to using either phosphatidylserine or phosphatidylserine coated liposomes instead of $A C$ to study the effects of $A C$ on inflammation. phosphatidylserine is used to replicate the main characteristic of $A C$, having phosphatidylserine located on the cell surface. While this may be a more convenient method, it does not take into account the other mediators AC produce when undergoing apoptosis. Here we show both apoptotic Jurkat cells (Figure 20) and apoptotic neutrophils (Figure 25) secrete factors that can alter macrophage cytokine production. Additionally, secreted factors from apoptotic Jurkat cells were able to decrease macrophage phagocytosis of $B$. burgdorferi (Figure 21A) and suppress neutrophil migration to $\mathrm{LTB}_{4}$ (Figure 21C). This demonstrates that factors besides phosphatidylserine on the cell surface can alter cytokine production macrophages and effector functions of neutrophils and macrophages.

While the use of phosphatidylserine may elicit responses, it does not take into account all of the secreted factors apoptotic cells produce. While it is known phosphatidylserine is an important characteristic of $A C$, we are still determining the role of other mediators, including actively secreted factors, that may contribute to the role these cells play during an inflammatory response. Additionally, some of these secreted factors may be responsible for or aid in the downregulation of inflammation, therefore, using $A C$ is more biologically relevant than using phosphatidylserine alone. Apoptosis is a biologically active process, 
meaning cells are undergoing physical changes and secreting various factors as apoptosis occurs, including cytokines and lipid mediators. Without these factors present, the full picture of how these cells are able to perform their antiinflammatory functions is incomplete.

All AC have similar characteristics, but different sources of AC may secrete different factors. Because the cells themselves are removed from the equation by only utilizing $\mathrm{AC}$ supernatant, what is causing the alteration in immune cell function must be something they produce and secrete. Apoptotic Jurkat cell supernatant was only able to alter macrophage cytokine production of TNF- $\alpha$ and did not affect KC or IL-10 production (Figure 20). However, when apoptotic neutrophil secretions were used, they were able to alter both TNF- $\alpha$ and IL-10 levels (Figure 25). This suggests differences between the mediators being secreted by apoptotic Jurkat cells and apoptotic neutrophils. The differences in these factors could potentially be cytokines or lipids, as we have shown apoptotic neutrophils can produce various cytokines (Figure 26) and PGE (Figure 28B). Cytokines can alter macrophage cytokine production and effector functions of both neutrophils and macrophages. Additionally, various inflammatory lipids are known to alter these functions in neutrophils and macrophages as well, so it seems plausible apoptotic Jurkat cells and apoptotic neutrophils may have different amounts of various secretory products. 
We have shown the injection of apoptotic Jurkat cells into the ankle joints of $B$. burgdorferi-infected mice decreased ankle swelling and promoted resolution

(Figure 4), but we have still not determined if the injection of apoptotic Jurkat cell or apoptotic neutrophil supernatant might elicit a similar effect. Since the ultimate goal for treatment associated with $\mathrm{AC}$ mechanisms is to develop a cell free therapeutic, determining the components in the $\mathrm{AC}$ supernatant responsible for the downregulation of inflammation could lead to another potential therapeutic approach. While the administration of cytokines would be fairly easy as they are administered during other diseases, treatment using lipid mediators might pose a problem. These often have a very short half-life in vivo, for example, $\mathrm{PGE}_{2}$ which has the half-life of only a few minutes [172], but others have modified these mediators to elicit their effects without such fast degradation [173].

\section{B. burgdorferi activated apoptotic neutrophils on macrophage functions in}

\section{vitro}

Even though apoptotic Jurkat cells and apoptotic neutrophils were able to alter macrophage function in vitro, we wanted to better replicate in vivo conditions, as far as the timing of bacterial administration. During an infection, neutrophils are first recruited to the site of infection to phagocytose $B$. burgdorferi and then undergo apoptosis. This means apoptotic neutrophils in vivo are more likely to have phagocytosed B. burgdorferi prior to undergoing apoptosis and subsequent efferocytosis. By activating neutrophils with $B$. burgdorferi prior to apoptosis induction, this will better replicate the order which B. burgdorferi phagocytosis, 
apoptosis, and efferocytosis normally occurs. As shown in this dissertation, this sequence of events results in an even more extreme alteration in cytokine production by macrophages (Figure 23) and was also true for E. coli-activated apoptotic neutrophils (Figure 24). This was the first example of activating neutrophils prior to apoptosis and then administering them to $B$. burgdorferi-cocultured macrophages.

While we have shown the effects of using activated apoptotic neutrophils in vitro, we have not yet determined why these cells have much more enhanced effects over unstimulated apoptotic neutrophils. Since activated apoptotic neutrophil supernatant also causes this alteration (Figure 25), it is likely a secreted factor is responsible. One possibility could be an alteration in apoptotic neutrophil cytokine production. Neutrophils make cytokines in response to a bacterial encounter, just as macrophages do, however, they generally make much lower levels of cytokines. In addition, of the cytokines we measured from activated apoptotic neutrophils, both pro- and anti-inflammatory mediators were higher, except for TNF- $\alpha$ (Figure 26). While other cytokines could be responsible for their effects on macrophages, it seems activated apoptotic neutrophils make higher levels of most cytokines overall, making cytokines an unlikely contributor to macrophage cytokine production.

Another probable contributor to this phenomenon is lipid mediators. Dendritic cells have enhanced production of $\mathrm{PGE}_{2}$ in response to efferocytosis of $E$. coli- 
infected macrophages, aiding in the downregulation of inflammation [57], and apoptotic Jurkat cells produce and enhance $B$. burgdorferi-co-cultured macrophage production of $\mathrm{PGE}_{2}$ as well (Figure 28). This means activated apoptotic neutrophils could produce enhanced $\mathrm{PGE}_{2}$ production in addition to other lipid mediators. Lipidomics analysis will be required on the apoptotic neutrophils and activated apoptotic neutrophils to determine what lipids are normally produced by apoptotic neutrophils and to determine if activated apoptotic neutrophils have an altered lipid production profile. There is still much to be done to fully understand the role of lipid mediators during apoptosis and efferocytosis.

\section{Apoptotic cells for therapeutic strategies}

Current therapeutic strategies for chronic inflammatory diseases mostly involves trying to suppress or prevent pro-inflammatory mediators. While this may temporarily alleviate the symptoms, it does not aid in inflammation resolution, meaning long-term therapeutics are often the only solution to these problems. As shown here, AC play a role during inflammation resolution, and their administration in B. burgdorferi-infected mice promotes earlier resolution. While the injection of $A C$ promotes resolution, we sought to determine the mechanism as to why this phenomenon occurs to be able to develop a cell free therapeutic strategy. To do this, we needed to determine the mechanism by which this phenomenon associated with $\mathrm{AC}$ and the downregulation of inflammation occurred. Previously, others have shown AC promote PPAR- $\psi$ expression, which 
aids in resolution during an inflammatory response [5, 77], however, we demonstrate a cell free mechanism in which we administered the PPAR- $\gamma$ agonist rosiglitazone, in lieu of AC, which was shown to have a similar effect (Figure 11). We also showed supernatant from apoptotic Jurkat cells (Figure 20) and activated apoptotic neutrophils (Figure 25) were able to alter macrophage cytokine production similar to AC, showing another potential cell free therapeutic. Furthering our understanding of this mechanism of how AC promote inflammation resolution will lead to better therapeutics strategies to resolve inflammation, rather than trying to block the pro-inflammatory mediators involved, which may prolong inflammation.

\section{Conclusions}

The work shown in this thesis represents substantial progress in understanding the role of apoptosis and apoptotic cells on inflammation resolution during experimental Lyme arthritis. We show the localized administration of apoptotic Jurkat cells into the tibio-tarsal joints of $B$. burgdorferi-infected mice aids in inflammation resolution by increasing PPAR- $\gamma$ activation. While others had shown the correlation between AC administration and PPAR-y [5], this is the first example of this during a bacterial infection and the use of localized rosiglitazone administration. We also show the importance of inflammatory lipid mediator signaling of the eicosanoids $\mathrm{LTB}_{4}$ and $\mathrm{PGE}_{2}$. While $\mathrm{LTB}_{4}$ signaling through $\mathrm{BLT1}$ is required for neutrophil apoptosis and clearance, our data suggests macrophages need exogenous $\mathrm{PGE}_{2}$ production for efferocytosis of apoptotic 
neutrophils. We also show the importance of using Jurkat cells and neutrophils as apoptotic cells instead of just phosphatidylserine, because these apoptotic cells are actively secreting cytokines and lipid mediators during their apoptosis, contributing to their role in the downregulation of inflammation. Utilizing activated apoptotic neutrophils, we were able to better replicate in vivo conditions, which enhanced the alteration of macrophage cytokine production, showing another aspect of this phenomenon that requires further understanding. Future studies will focus on why macrophages require production of $P \mathrm{PE}_{2}$ for cytokine alteration by apoptotic neutrophils and efferocytosis. In addition, we will determine the secretory components of both apoptotic neutrophils and activated apoptotic neutrophils to determine what component in their supernatant is resulting in their anti-inflammatory effects on macrophages and to determine why activated apoptotic neutrophils have an enhanced ability over unstimulated apoptotic neutrophils. 


\section{References}

1. Fullerton, J.N. and D.W. Gilroy, Resolution of inflammation: a new therapeutic frontier. Nat Rev Drug Discov, 2016. 15(8): p. 551-567.

2. Ritzman, A.M., et al., The chemokine receptor CXCR2 ligand KC (CXCL1) mediates neutrophil recruitment and is critical for development of experimental Lyme arthritis and carditis. Infect Immun, 2010. 78(11): p. 4593-4600.

3. Blaho, V.A., et al., 5-Lipoxygenase-deficient mice infected with Borrelia burgdorferi develop persistent arthritis. J Immunol, 2011. 186(5): p. 30763084.

4. Buckley, C.D., D.W. Gilroy, and C.N. Serhan, Proresolving lipid mediators and mechanisms in the resolution of acute inflammation. Immunity, 2014. 40(3): p. 315-327.

5. Yoon, Y.S., et al., PPARgamma activation following apoptotic cell instillation promotes resolution of lung inflammation and fibrosis via regulation of efferocytosis and proresolving cytokines. Mucosal Immunol, 2015. 8(5): p. 1031-1046.

6. Hilliard, K.A. and C.R. Brown, Treatment of Borrelia burgdorferi-infected mice with apoptotic cells attenuates Lyme arthritis via PPAR-gamma. J Immunol, 2019.

7. Kovalenko, A., et al., The tumour suppressor CYLD negatively regulates NF-kappaB signalling by deubiquitination. Nature, 2003. 424(6950): p. 801-805. 
8. Degraaf, A.J., et al., Prostaglandin E2 reduces Toll-like receptor 4 expression in alveolar macrophages by inhibition of translation. Am J Respir Cell Mol Biol, 2014. 51(2): p. 242-250.

9. O'Brien, A.J., et al., Immunosuppression in acutely decompensated cirrhosis is mediated by prostaglandin E2. Nat Med, 2014. 20(5): p. 518523.

10. Abdulkhaleq, L.A., et al., The crucial roles of inflammatory mediators in inflammation: A review. Vet World, 2018. 11(5): p. 627-635.

11. Alessandri, A.L., et al., Resolution of inflammation: mechanisms and opportunity for drug development. Pharmacol Ther, 2013. 139(2): p. 189212.

12. Murakami, M. and T. Hirano, The molecular mechanisms of chronic inflammation development. Front Immunol, 2012. 3: p. 323-336.

13. Nathan, C. and A. Ding, Nonresolving inflammation. Cell, 2010. 140(6): p. 871-882.

14. Tait, S.W., G. Ichim, and D.R. Green, Die another way--non-apoptotic mechanisms of cell death. J Cell Sci, 2014. 127(Pt 10): p. 2135-2144.

15. Lockshin, R.A. and C.M. Williams, Programmed cell death--I. Cytology of degeneration in the intersegmental muscles of the pernyi silkmoth. $\mathrm{J}$ Insect Physiol, 1965. 11: p. 123-133.

16. Nagata, S., Apoptosis and clearance of apoptotic cells. Annu Rev Immunol, 2018. 36: p. 489-517. 
17. Thorp, E., M. Subramanian, and I. Tabas, The role of macrophages and dendritic cells in the clearance of apoptotic cells in advanced atherosclerosis. Eur J Immunol, 2011. 41(9): p. 2515-2518.

18. Crowley, L.C. and N.J. Waterhouse, Detecting cleaved caspase-3 in apoptotic cells by flow cytometry. Cold Spring Harb Protoc, 2016. 2016(11).

19. Fadok, V.A., et al., Exposure of phosphatidylserine on the surface of apoptotic lymphocytes triggers specific recognition and removal by macrophages. J Immunol, 1992. 148(7): p. 2207-2216.

20. Segawa, K., et al., Caspase-mediated cleavage of phospholipid flippase for apoptotic phosphatidylserine exposure. Science, 2014. 344(6188): $p$. 1164-1168.

21. Walker, N.I., et al., Patterns of cell death. Methods Achiev Exp Pathol, 1988. 13: p. $18-54$.

22. Galluzzi, L. and G. Kroemer, Necroptosis turns TNF lethal. Immunity, 2011. 35(6): p. 849-851.

23. Cho, Y.S., et al., Phosphorylation-driven assembly of the RIP1-RIP3 complex regulates programmed necrosis and virus-induced inflammation. Cell, 2009. 137(6): p. 1112-1123.

24. Geserick, P., et al., Cellular IAPs inhibit a cryptic CD95-induced cell death by limiting RIP1 kinase recruitment. J Cell Biol, 2009. 187(7): p. 10371054. 
25. Zhang, D.W., et al., RIP3, an energy metabolism regulator that switches TNF-induced cell death from apoptosis to necrosis. Science, 2009. 325(5938): p. 332-336.

26. Kayagaki, N., et al., Non-canonical inflammasome activation targets caspase-11. Nature, 2011. 479(7371): p. 117-121.

27. Miao, E.A., et al., Caspase-1-induced pyroptosis is an innate immune effector mechanism against intracellular bacteria. Nat Immunol, 2010. 11(12): p. 1136-1142.

28. Bergsbaken, T., S.L. Fink, and B.T. Cookson, Pyroptosis: host cell death and inflammation. Nat Rev Microbiol, 2009. 7(2): p. 99-109.

29. Fink, S.L. and B.T. Cookson, Caspase-1-dependent pore formation during pyroptosis leads to osmotic lysis of infected host macrophages. Cell Microbiol, 2006. 8(11): p. 1812-1825.

30. Brinkmann, V., et al., Neutrophil extracellular traps kill bacteria. Science, 2004. 303(5663): p. 1532-1535.

31. Brinkmann, V. and A. Zychlinsky, Beneficial suicide: why neutrophils die to make NETs. Nat Rev Microbiol, 2007. 5(8): p. 577-582.

32. Fuchs, T.A., et al., Novel cell death program leads to neutrophil extracellular traps. J Cell Biol, 2007. 176(2): p. 231-241.

33. Remijsen, Q., et al., Neutrophil extracellular trap cell death requires both autophagy and superoxide generation. Cell Res, 2011. 21(2): p. 290-304.

34. Roghanian, A. and J.M. Sallenave, Neutrophil elastase (NE) and NE inhibitors: canonical and noncanonical functions in lung chronic 
inflammatory diseases (cystic fibrosis and chronic obstructive pulmonary disease). J Aerosol Med Pulm Drug Deliv, 2008. 21(1): p. 125-144.

35. Loreto, C., et al., The role of intrinsic pathway in apoptosis activation and progression in Peyronie's disease. Biomed Res Int, 2014. 2014.

36. Rowinsky, E.K., Targeted induction of apoptosis in cancer management: the emerging role of tumor necrosis factor-related apoptosis-inducing ligand receptor activating agents. J Clin Oncol, 2005. 23(36): p. 93949407.

37. Strasser, A., P.J. Jost, and S. Nagata, The many roles of FAS receptor signaling in the immune system. Immunity, 2009. 30(2): p. 180-192.

38. Walczak, H., Death receptor-ligand systems in cancer, cell death, and inflammation. Cold Spring Harb Perspect Biol, 2013. 5(5).

39. Lavrik, I.N. and P.H. Krammer, Regulation of CD95/Fas signaling at the DISC. Cell Death Differ, 2012. 19(1): p. 36-41.

40. Lauber, K., et al., Clearance of apoptotic cells: getting rid of the corpses. Mol Cell, 2004. 14: p. 277-287.

41. Gregory, C., Cell biology: Sent by the scent of death. Nature, 2009. 461(7261): p. 181-182.

42. Grimsley, C. and K.S. Ravichandran, Cues for apoptotic cell engulfment: eat-me, don't eat-me and come-get-me signals. Trends Cell Biol, 2003. 13(12): p. 648-656.

43. Savill, J., Apoptosis in resolution of inflammation. J Leukoc Biol, 1997. 61(4): p. 375-380. 
44. Ravichandran, K.S., Find-me and eat-me signals in apoptotic cell clearance: progress and conundrums. J Exp Med, 2010. 207(9): p. 18071817.

45. Hawkins, L.A. and A. Devitt, Current understanding of the mechanisms for clearance of apoptotic cells-a fine balance. J Cell Death, 2013. 6: p. 5768.

46. Elliott, M.R., et al., Nucleotides released by apoptotic cells act as a findme signal to promote phagocytic clearance. Nature, 2009. 461(7261): $p$. 282-286.

47. Zitvogel, L., O. Kepp, and G. Kroemer, Decoding cell death signals in inflammation and immunity. Cell, 2010. 140(6): p. 798-804.

48. Greenberg, M.E., et al., Oxidized phosphatidylserine-CD36 interactions play an essential role in macrophage-dependent phagocytosis of apoptotic cells. J Exp Med, 2006. 203(12): p. 2613-2625.

49. Ramprasad, M.P., et al., The 94- to 97-kDa mouse macrophage membrane protein that recognizes oxidized low density lipoprotein and phosphatidylserine-rich liposomes is identical to macrosialin, the mouse homologue of human CD68. Proc Natl Acad Sci U S A, 1995. 92(21): $p$. 9580-9584.

50. Murphy, J.E., et al., LOX-1 scavenger receptor mediates calciumdependent recognition of phosphatidylserine and apoptotic cells. Biochem J, 2006. 393(Pt 1): p. 107-115. 
51. Gardai, S.J., et al., Cell-surface calreticulin initiates clearance of viable or apoptotic cells through trans-activation of LRP on the phagocyte. Cell, 2005. 123(2): p. 321-334.

52. Simhadri, V.R., et al., Human CD300a binds to phosphatidylethanolamine and phosphatidylserine, and modulates the phagocytosis of dead cells. Blood, 2012. 119(12): p. 2799-2809.

53. Brown, S., et al., Apoptosis disables CD31-mediated cell detachment from phagocytes promoting binding and engulfment. Nature, 2002. 418(6894): p. 200-203.

54. Devitt, A., et al., Human CD14 mediates recognition and phagocytosis of apoptotic cells. Nature, 1998. 392(6675): p. 505-509.

55. McNeil, P.L., et al., Acidification of phagosomes is initiated before lysosomal enzyme activity is detected. J Cell Biol, 1983. 97(3): p. 692-702.

56. Voll, R.E., et al., Immunosuppressive effects of apoptotic cells. Nature, 1997. 390(6658): p. 350-351.

57. Dejani, N.N., et al., Intestinal host defense outcome is dictated by PGE2 production during efferocytosis of infected cells. Proc Natl Acad Sci U S A, 2018. 115(36): p. E8469-E8478.

58. Laguna, R.K., et al., A Legionella pneumophila-translocated substrate that is required for growth within macrophages and protection from host cell death. Proc Natl Acad Sci U S A, 2006. 103(49): p. 18745-18750. 
59. Banga, S., et al., Legionella pneumophila inhibits macrophage apoptosis by targeting pro-death members of the Bcl2 protein family. Proc Natl Acad Sci U S A, 2007. 104(12): p. 5121-5126.

60. Rikihisa, Y., Anaplasma phagocytophilum and Ehrlichia chaffeensis: subversive manipulators of host cells. Nat Rev Microbiol, 2010. 8(5): p. 328-339.

61. Mimuro, H., et al., Helicobacter pylori dampens gut epithelial self-renewal by inhibiting apoptosis, a bacterial strategy to enhance colonization of the stomach. Cell Host Microbe, 2007. 2(4): p. 250-263.

62. Loeuillet, C., et al., Mycobacterium tuberculosis subverts innate immunity to evade specific effectors. J Immunol, 2006. 177(9): p. 6245-6255.

63. Lindemans, C.A., et al., Respiratory syncytial virus inhibits granulocyte apoptosis through a phosphatidylinositol 3-kinase and NF-kappaBdependent mechanism. J Immunol, 2006. 176(9): p. 5529-5537.

64. Skaletskaya, A., et al., A cytomegalovirus-encoded inhibitor of apoptosis that suppresses caspase-8 activation. Proc Natl Acad Sci U S A, 2001. 98(14): p. 7829-7834.

65. Pitrak, D.L., et al., Accelerated neutrophil apoptosis in the acquired immunodeficiency syndrome. J Clin Invest, 1996. 98(12): p. 2714-2719.

66. Lichtner, M., et al., HIV protease inhibitor therapy reverses neutrophil apoptosis in AIDS patients by direct calpain inhibition. Apoptosis, 2006. 11(5): p. 781-787. 
67. Colamussi, M.L., et al., Influenza A virus accelerates neutrophil apoptosis and markedly potentiates apoptotic effects of bacteria. Blood, 1999. 93(7): p. 2395-2403.

68. Fadeel, B. and S. Orrenius, Apoptosis: a basic biological phenomenon with wide-ranging implications in human disease. J Intern Med, 2005. 258(6): p. 479-517.

69. Singh, N., Apoptosis in health and disease and modulation of apoptosis for therapy: An overview. Indian J Clin Biochem, 2007. 22(2): p. 6-16.

70. Ren, Y., et al., Increased apoptotic neutrophils and macrophages and impaired macrophage phagocytic clearance of apoptotic neutrophils in systemic lupus erythematosus. Arthritis Rheum, 2003. 48(10): p. 28882897.

71. Yang, Y., et al., Programmed cell death and its role in inflammation. Mil Med Res, 2015. 2: p. 12.

72. Henriksen, P.A., et al., Gene delivery of the elastase inhibitor elafin protects macrophages from neutrophil elastase-mediated impairment of apoptotic cell recognition. FEBS Lett, 2004. 574: p. 80-84.

73. Sanchez, I., et al., Caspase-8 is required for cell death induced by expanded polyglutamine repeats. Neuron, 1999. 22(3): p. 623-633.

74. Lambrechts, D., et al., VEGF is a modifier of amyotrophic lateral sclerosis in mice and humans and protects motoneurons against ischemic death. Nat Genet, 2003. 34(4): p. 383-394. 
75. Fulda, S. and K.M. Debatin, Targeting apoptosis pathways in cancer therapy. Curr Cancer Drug Targets, 2004. 4(7): p. 569-576.

76. Henson, P.M. and D.L. Bratton, Antiinflammatory effects of apoptotic cells. J Clin Invest, 2013. 123(7): p. 2773-2774.

77. Perruche, S., P. Saas, and W. Chen, Apoptotic cell-mediated suppression of streptococcal cell wall-induced arthritis is associated with alteration of macrophage function and local regulatory T-cell increase: a potential cellbased therapy? Arthritis Res Ther, 2009. 11(4): p. R104.

78. Petelin, Z., et al., CD95/Fas expression on peripheral blood T lymphocytes in patients with multiple sclerosis: effect of high-dose methylprednisolone therapy. Clin Neurol Neurosurg, 2004. 106(3): p. 259-262.

79. Reed, J.C. and M. Pellecchia, Apoptosis-based therapies for hematologic malignancies. Blood, 2005. 106(2): p. 408-418.

80. CDC. Lyme Disease, on Centers for Disease Control and Prevention. 2016; Available from: http://www.cdc.gov/lyme/.

81. Eisen, R.J., L. Eisen, and C.B. Beard, County-scale distribution of Ixodes scapularis and Ixodes pacificus (Acari: Ixodidae) in the continental United States. J Med Entomol, 2016. 53(2): p. 349-386.

82. Kugeler, K.J., et al., Geographic distribution and expansion of human Lyme disease, United States. Emerg Infect Dis, 2015. 21(8): p. 14551457. 
83. Feng, J., et al., Identification of novel activity against Borrelia burgdorferi persisters using an FDA approved drug library. Emerg Microbes Infect, 2014. 3(7): p. e49.

84. Radolf, J.D., et al., Of ticks, mice and men: understanding the dual-host lifestyle of Lyme disease spirochaetes. Nat Rev Microbiol, 2012. 10(2): $\mathrm{p}$. 87-99.

85. Kurtenbach, K., et al., Fundamental processes in the evolutionary ecology of Lyme borreliosis. Nat Rev Microbiol, 2006. 4(9): p. 660-669.

86. Tilly, K., P.A. Rosa, and P.E. Stewart, Biology of infection with Borrelia burgdorferi. Infect Dis Clin North Am, 2008. 22(2): p. 217-234.

87. Shih, C.M., et al., Delayed dissemination of Lyme disease spirochetes from the site of deposition in the skin of mice. Journal of Infectious Disease, 1992. 166(4): p. 827-831.

88. Hutton, T.A., et al., Search for Borrelia burgdorferi in kidneys of dogs with suspected "Lyme nephritis". J Vet Intern Med, 2008. 22(4): p. 860-865.

89. Dandache, P. and R.B. Nadelman, Erythema migrans. Infect Dis Clin North Am, 2008. 22(2): p. 235-260.

90. Wormser, G.P., et al., The clinical assessment, treatment, and prevention of Lyme disease, human granulocytic anaplasmosis, and babesiosis: clinical practice guidelines by the infectious diseases society of america. Clin Infect Dis, 2006. 43(9): p. 1089-1134.

91. de Koning, J., et al., Demonstration of spirochetes in cardiac biopsies of patients with Lyme disease. J Infect Dis, 1989. 160(1): p. 150-153. 
92. Sharma, B., et al., Borrelia burgdorferi, the causative agent of Lyme disease, forms drug-tolerant persister cells. Antimicrob Agents Chemother, 2015. 59(8): p. 4616-4624.

93. Steere, A.C., Lyme disease. New England Journal of Medicine, 2001. 345(2): p. 115-125.

94. Barthold, S.W., M. deSouza, and S. Feng, Serum-mediated resolution of Lyme arthritis in mice. Lab Invest, 1996. 74(1): p. 57-67.

95. Brown, C.R. and S.L. Reiner, Activation of natural killer cells in arthritissusceptible but not arthritis-resistant mouse strains following Borrelia burgdorferi infection. Infect Immun, 1998. 66(11): p. 5208-5214.

96. Brown, C.R. and S.L. Reiner, Clearance of Borrelia burgdorferi may not be required for resistance to experimental Lyme arthritis. Infect Immun, 1998. 66(5): p. 2065-2071.

97. Lasky, C.E., et al., Infection of interleukin 17 Receptor A-deficient C3H mice with Borrelia burgdorferi does not affect their development of Lyme arthritis and carditis. Infect Immun, 2015. 83(7): p. 2882-2888.

98. Lasky, C.E., et al., T cells exacerbate Lyme Borreliosis in TLR2-deficient mice. Front Immunol, 2016. 7: p. 468-474.

99. Brown, C.R. and S.L. Reiner, Genetic control of experimental Lyme arthritis in the absence of specific immunity. Infect Immun, 1999. 67(4): p. 1967-1973.

100. Brown, C.R., V.A. Blaho, and C.M. Loiacono, Susceptibility to experimental Lyme arthritis correlates with KC and monocyte 
chemoattractant protein-1 production in joints and requires neutrophil recruitment via CXCR2. J Immunol, 2003. 171(2): p. 893-901.

101. Anguita, J., et al., Selective anti-inflammatory action of interleukin-11 in murine Lyme disease: arthritis decreases while carditis persists. J Infect Dis, 1999. 179(3): p. 734-737.

102. Anguita, J., et al., Effect of anti-interleukin 12 treatment on murine Lyme borreliosis. J Clin Invest, 1996. 97(4): p. 1028-1034.

103. Anguita, J., et al., Borrelia burgdorferi-infected, interleukin-6-deficient mice have decreased Th2 responses and increased Lyme arthritis. J Infect Dis, 1998. 178(5): p. 1512-1515.

104. Blaho, V.A., W.J. Mitchell, and C.R. Brown, Arthritis develops but fails to resolve during inhibition of cyclooxygenase 2 in a murine model of Lyme disease. Arthritis Rheum, 2008. 58(5): p. 1485-1495.

105. Stables, M.J. and D.W. Gilroy, Old and new generation lipid mediators in acute inflammation and resolution. Prog Lipid Res, 2011. 50(1): p. 35-51.

106. Dennis, E.A. and P.C. Norris, Eicosanoid storm in infection and inflammation. Nat Rev Immunol, 2015. 15(8): p. 511-523.

107. Shinomiya, S., et al., Regulation of TNF-alpha and interleukin-10 production by prostaglandins I(2) and E(2): studies with prostaglandin receptor-deficient mice and prostaglandin E-receptor subtype-selective synthetic agonists. Biochem Pharmacol, 2001. 61(9): p. 1153-1160. 
108. Blaho, V.A., et al., Lipidomic analysis of dynamic eicosanoid responses during the induction and resolution of Lyme arthritis. J Biol Chem, 2009. 284(32): p. 21599-21612.

109. Barthold, S.W., et al., Experimental Lyme arthritis in rats infected with Borrelia burgdorferi. J Infect Dis, 1988. 157(4): p. 842-846.

110. Carroll, J.A., et al., An enhanced GFP reporter system to monitor gene expression in Borrelia burgdorferi. Microbiology, 2003. 149(7): p. 18191828.

111. Lokuta, M.A., P.A. Nuzzi, and A. Huttenlocher, Analysis of neutrophil polarization and chemotaxis. Methods Mol Biol, 2007. 412: p. 211-229.

112. Lasky, C.E., R.M. Olson, and C.R. Brown, Macrophage polarization during murine Lyme borreliosis. Infect Immun, 2015. 83(7): p. 2627-2635.

113. Montgomery, R.R., et al., Human phagocytic cells in the early innate immune response to Borrelia burgdorferi. J Infect Dis, 2002. 185(12): p. 1773-1779.

114. Xu, Q., et al., Increasing the recruitment of neutrophils to the site of infection dramatically attenuates Borrelia burgdorferi infectivity. J Immunol, 2007. 178(8): p. 5109-5115.

115. Jiang, C., A.T. Ting, and B. Seed, PPAR-gamma agonists inhibit production of monocyte inflammatory cytokines. Nature, 1998. 391(6662): p. 82-86. 
116. Pascual, G., et al., A SUMOylation-dependent pathway mediates transrepression of inflammatory response genes by PPAR-gamma. Nature, 2005. 437(7059): p. 759-763.

117. Reddy, R.C., et al., Sepsis-induced inhibition of neutrophil chemotaxis is mediated by activation of peroxisome proliferator-activated receptor\{gamma\}. Blood, 2008. 112(10): p. 4250-4258.

118. Fadok, V.A., et al., Macrophages that have ingested apoptotic cells in vitro inhibit proinflammatory cytokine production through autocrine/paracrine mechanisms involving TGF-beta, PGE2, and PAF. J Clin Invest, 1998. $101(4):$ p. 890-898.

119. Brown, C.R., V.A. Blaho, and C.M. Loiacono, Treatment of mice with the neutrophil-depleting antibody RB6-8C5 results in early development of experimental Lyme arthritis via the recruitment of Gr-1polymorphonuclear leukocyte-like cells. Infect Immun, 2004. 72(9): p. 4956-4965.

120. Huynh, M.L., V.A. Fadok, and P.M. Henson, Phosphatidylserinedependent ingestion of apoptotic cells promotes TGF-beta1 secretion and the resolution of inflammation. J Clin Invest, 2002. 109(1): p. 41-50.

121. Araujo, C.V., et al., A PPARgamma agonist enhances bacterial clearance through neutrophil extracellular trap formation and improves survival in sepsis. Shock, 2016. 45(4): p. 393-403. 
122. Fox, S., et al., Neutrophil apoptosis: relevance to the innate immune response and inflammatory disease. J Innate Immun, 2010. 2(3): p. 216227.

123. Silva, M.T., Bacteria-induced phagocyte secondary necrosis as a pathogenicity mechanism. J Leukoc Biol, 2010. 88(5): p. 885-896.

124. Griffiths, R.J., et al., Collagen-induced arthritis is reduced in 5lipoxygenase-activating protein-deficient mice. J Exp Med, 1997. 185(6): p. $1123-1129$.

125. Peters-Golden, M. and T.G. Brock, 5-lipoxygenase and FLAP. Prostaglandins Leukot Essent Fatty Acids, 2003. 69(2-3): p. 99-109.

126. Serhan, C.N. and B.D. Levy, Resolvins in inflammation: emergence of the pro-resolving superfamily of mediators. J Clin Invest, 2018. 128(7): p. 2657-2669.

127. Chan, M.M. and A.R. Moore, Resolution of inflammation in murine autoimmune arthritis is disrupted by cyclooxygenase-2 inhibition and restored by prostaglandin E2-mediated lipoxin A4 production. J Immunol, 2010. 184(11): p. 6418-6426.

128. Zhang, L., et al., BML-111, a lipoxin receptor agonist, modulates the immune response and reduces the severity of collagen-induced arthritis. Inflamm Res, 2008. 57(4): p. 157-162.

129. Norling, L.V., et al., Proresolving and cartilage-protective actions of resolvin D1 in inflammatory arthritis. JCI Insight, 2016. 1(5): p. e85922. 
130. Arnardottir, H.H., et al., Resolvin D3 Is Dysregulated in Arthritis and Reduces Arthritic Inflammation. J Immunol, 2016. 197(6): p. 2362-2368.

131. Saeki, K. and T. Yokomizo, Identification, signaling, and functions of LTB4 receptors. Semin Immunol, 2017. 33: p. 30-36.

132. Serhan, C.N. and N.A. Petasis, Resolvins and protectins in inflammation resolution. Chem Rev, 2011. 111(10): p. 5922-5943.

133. Janicke, R.U., et al., Caspase-3 is required for alpha-fodrin cleavage but dispensable for cleavage of other death substrates in apoptosis. J Biol Chem, 1998. 273(25): p. 15540-15545.

134. Zhang, Y., R.M. Olson, and C.R. Brown, Macrophage LTB4 drives efficient phagocytosis of Borrelia burgdorferi via BLT1 or BLT2. J Lipid Res, 2017. 58(3): p. 494-503.

135. Subbarao, K., et al., Role of leukotriene B4 receptors in the development of atherosclerosis: potential mechanisms. Arterioscler Thromb Vasc Biol, 2004. 24(2): p. 369-375.

136. Arita, M., et al., Resolvin E1 selectively interacts with leukotriene B4 receptor BLT1 and ChemR23 to regulate inflammation. J Immunol, 2007. 178(6): p. 3912-3917.

137. El Kebir, D., P. Gjorstrup, and J.G. Filep, Resolvin E1 promotes phagocytosis-induced neutrophil apoptosis and accelerates resolution of pulmonary inflammation. Proc Natl Acad Sci U S A, 2012. 109(37): p. 14983-14988. 
138. Haworth, O., et al., Resolvin E1 regulates interleukin 23, interferongamma and lipoxin A4 to promote the resolution of allergic airway inflammation. Nat Immunol, 2008. 9(8): p. 873-879.

139. Arita, M., et al., Stereochemical assignment, antiinflammatory properties, and receptor for the omega-3 lipid mediator resolvin E1. J Exp Med, 2005. 201(5): p. 713-722.

140. Fredman, G., et al., Impaired phagocytosis in localized aggressive periodontitis: rescue by Resolvin E1. PLoS One, 2011. 6(9): p. e24422.

141. Bhattacharjee, R., et al., cAMP prevents TNF-induced apoptosis through inhibiting DISC complex formation in rat hepatocytes. Biochem Biophys Res Commun, 2012. 423(1): p. 85-90.

142. Park, Y.H., M.S. Jeong, and S.B. Jang, Structural insights of homotypic interaction domains in the ligand-receptor signal transduction of tumor necrosis factor (TNF). BMB Rep, 2016. 49(3): p. 159-166.

143. Wei, L., D. Ding, and R. Salvi, Salicylate-induced degeneration of cochlea spiral ganglion neurons-apoptosis signaling. Neuroscience, 2010. 168(1): p. 288-299.

144. Leo, E., et al., TRAF1 is a substrate of caspases activated during tumor necrosis factor receptor-alpha-induced apoptosis. J Biol Chem, 2001. 276(11): p. 8087-8093.

145. Dasgupta, Y., et al., Normal ABL1 is a tumor suppressor and therapeutic target in human and mouse leukemias expressing oncogenic $A B L 1$ kinases. Blood, 2016. 127(17): p. 2131-2143. 
146. Zhang, M., et al., Lack of TNF-alpha promotes caspase-3-independent apoptosis during murine cytomegalovirus retinitis. Invest Ophthalmol Vis Sci, 2011. 52(3): p. 1800-1808.

147. DeLeon-Pennell, K.Y., et al., CD36 Is a Matrix Metalloproteinase-9 Substrate That Stimulates Neutrophil Apoptosis and Removal During Cardiac Remodeling. Circ Cardiovasc Genet, 2016. 9(1): p. 14-25.

148. Soares, E.M., et al., Leukotriene B4 enhances innate immune defense against the puerperal sepsis agent Streptococcus pyogenes. J Immunol, 2013. 190(4): p. 1614-1622.

149. Perez-Garijo, A., Y. Fuchs, and H. Steller, Apoptotic cells can induce nonautonomous apoptosis through the TNF pathway. Elife, 2013. 2: p. e01004.

150. Mora, J., et al., Interleukin-38 is released from apoptotic cells to limit inflammatory macrophage responses. J Mol Cell Biol, 2016. 8(5): p. 426438.

151. Hangai, S., et al., PGE2 induced in and released by dying cells functions as an inhibitory DAMP. Proc Natl Acad Sci U S A, 2016. 113(14): p. 38443849.

152. Jondle, C.N., et al., Klebsiella pneumoniae infection of murine neutrophils impairs their efferocytic clearance by modulating cell death machinery. PLoS Pathog, 2018. 14(10): p. e1007338.

153. Ramos, G.C., et al., Apoptotic mimicry: phosphatidylserine liposomes reduce inflammation through activation of peroxisome proliferator- 
activated receptors (PPARs) in vivo. $\mathrm{Br} \mathrm{J}$ Pharmacol, 2007. 151(6): $\mathrm{p}$. 844-850.

154. Murakami, Y., et al., CD300b regulates the phagocytosis of apoptotic cells via phosphatidylserine recognition. Cell Death Differ, 2014. 21(11): p. 1746-1757.

155. Brock, T.G., R.W. McNish, and M. Peters-Golden, Arachidonic acid is preferentially metabolized by cyclooxygenase-2 to prostacyclin and prostaglandin E2. J Biol Chem, 1999. 274(17): p. 11660-11666.

156. Akaogi, J., et al., Role of PGE2 and EP receptors in the pathogenesis of rheumatoid arthritis and as a novel therapeutic strategy. Endocr Metab Immune Disord Drug Targets, 2006. 6(4): p. 383-394.

157. Wahli, W. and L. Michalik, PPARs at the crossroads of lipid signaling and inflammation. Trends Endocrinol Metab, 2012. 23(7): p. 351-363.

158. Dey, I., M. Lejeune, and K. Chadee, Prostaglandin E2 receptor distribution and function in the gastrointestinal tract. Br J Pharmacol, 2006. 149(6): $\mathrm{p}$. 611-623.

159. Brauchle, E., et al., Cell death stages in single apoptotic and necrotic cells monitored by Raman microspectroscopy. Sci Rep, 2014. 4(4698).

160. Gray, M., et al., Apoptotic cells protect mice from autoimmune inflammation by the induction of regulatory B cells. Proc Natl Acad Sci U S A, 2007. 104(35): p. 14080-14085. 
161. Bonnefoy, F., et al., Apoptotic cell infusion treats ongoing collageninduced arthritis, even in the presence of methotrexate, and is synergic with anti-TNF therapy. Arthritis Res Ther, 2016. 18(1): p. 184.

162. Tyagi, S., et al., The peroxisome proliferator-activated receptor: A family of nuclear receptors role in various diseases. J Adv Pharm Technol Res, 2011. 2(4): p. 236-240.

163. Hiatt, W.R., S. Kaul, and R.J. Smith, The cardiovascular safety of diabetes drugs--insights from the rosiglitazone experience. N Engl J Med, 2013. 369(14): p. 1285-1287.

164. Shao, W.H., et al., Targeted disruption of leukotriene B4 receptors BLT1 and BLT2: a critical role for BLT1 in collagen-induced arthritis in mice. J Immunol, 2006. 176(10): p. 6254-6261.

165. Chen, M., et al., Neutrophil-derived leukotriene B4 is required for inflammatory arthritis. J Exp Med, 2006. 203(4): p. 837-842.

166. Fattahi, M.J. and A. Mirshafiey, Prostaglandins and rheumatoid arthritis. Arthritis, 2012. 2012: p. 239310.

167. Kristo, F., et al., Pharmacological inhibition of BLT1 diminishes early abdominal aneurysm formation. Atherosclerosis, 2010. 210(1): p. 107-113.

168. Solomon, S.D., et al., Cardiovascular risk associated with celecoxib in a clinical trial for colorectal adenoma prevention. N Engl J Med, 2005. 352(11): p. 1071-1080.

169. Fitzgerald, G.A., Coxibs and cardiovascular disease. N Engl J Med, 2004. 351(17): p. 1709-1711. 
170. Boulet, L., et al., Deletion of microsomal prostaglandin E2 (PGE2) synthase-1 reduces inducible and basal PGE2 production and alters the gastric prostanoid profile. J Biol Chem, 2004. 279(22): p. 23229-23237.

171. Loynes, C.A., et al., PGE2 production at sites of tissue injury promotes an anti-inflammatory neutrophil phenotype and determines the outcome of inflammation resolution in vivo. Sci Adv, 2018. 4(9): p. eaar8320.

172. Kozak, K.R., et al., Metabolism of prostaglandin glycerol esters and prostaglandin ethanolamides in vitro and in vivo. J Biol Chem, 2001. 276(40): p. 36993-36998.

173. Serhan, C.N., Pro-resolving lipid mediators are leads for resolution physiology. Nature, 2014. 510(7503): p. 92-101. 


\section{Appendix I}

\begin{tabular}{|c|c|c|c|c|c|}
\hline \multirow[b]{2}{*}{ Symbol } & \multicolumn{2}{|c|}{$\begin{array}{c}\text { AVG } \Delta \mathrm{C}_{\mathrm{t}} \\
\text { (Ct(GOI) - Ave Ct (HKG)) }\end{array}$} & \multicolumn{2}{|c|}{$2^{\wedge}-\Delta C_{t}$} & \multirow{2}{*}{$\begin{array}{c}\begin{array}{c}\text { Fold Up- or Down- } \\
\text { Regulation }\end{array} \\
\begin{array}{c}\text { Test Group } \\
\text { /Control Group }\end{array}\end{array}$} \\
\hline & $\begin{array}{l}\text { Test } \\
\text { Group }\end{array}$ & $\begin{array}{l}\text { Control } \\
\text { Group }\end{array}$ & $\begin{array}{l}\text { Test } \\
\text { Group }\end{array}$ & $\begin{array}{l}\text { Control } \\
\text { Group }\end{array}$ & \\
\hline Abl1 & 7.10 & 5.83 & 7.3E-03 & $1.8 \mathrm{E}-02$ & -2.42 \\
\hline Aifm1 & 6.55 & 6.05 & 1.1E-02 & 1.5E-02 & -1.41 \\
\hline Akt1 & 5.82 & 5.54 & 1.8E-02 & 2.1E-02 & -1.21 \\
\hline Anxa5 & 5.59 & 5.01 & 2.1E-02 & 3.1E-02 & -1.50 \\
\hline Apaf1 & 6.74 & 6.04 & 9.3E-03 & 1.5E-02 & -1.63 \\
\hline Api5 & 5.13 & 5.00 & 2.9E-02 & 3.1E-02 & -1.09 \\
\hline Atf5 & 6.41 & 5.63 & 1.2E-02 & 2.0E-02 & -1.71 \\
\hline Bad & 6.18 & 6.00 & $1.4 \mathrm{E}-02$ & 1.6E-02 & -1.14 \\
\hline Bag1 & 6.39 & 6.31 & 1.2E-02 & 1.3E-02 & -1.06 \\
\hline Bag3 & 5.84 & 5.29 & 1.7E-02 & 2.6E-02 & -1.46 \\
\hline Bak1 & 6.51 & 6.95 & 1.1E-02 & 8.1E-03 & 1.36 \\
\hline Bax & 5.14 & 4.09 & 2.8E-02 & 5.9E-02 & -2.07 \\
\hline Bcl10 & 4.89 & 4.65 & 3.4E-02 & 4.0E-02 & -1.18 \\
\hline Bcl2 & 6.30 & 5.73 & 1.3E-02 & 1.9E-02 & -1.49 \\
\hline Bcl2a1a & 6.14 & 5.03 & $1.4 \mathrm{E}-02$ & 3.1E-02 & -2.16 \\
\hline Bcl2I1 & 3.71 & 2.38 & 7.6E-02 & 1.9E-01 & -2.52 \\
\hline Bcl2l10 & 7.15 & 6.86 & 7.0E-03 & 8.6E-03 & -1.22 \\
\hline $\mathrm{Bcl} 2 \mathrm{l} 11$ & 6.35 & 6.49 & 1.2E-02 & 1.1E-02 & 1.10 \\
\hline Bcl2I2 & 7.22 & 6.80 & 6.7E-03 & 9.0E-03 & -1.34 \\
\hline Bid & 6.78 & 6.53 & 9.1E-03 & 1.1E-02 & -1.19 \\
\hline Birc2 & 5.83 & 5.05 & 1.8E-02 & 3.0E-02 & -1.72 \\
\hline Birc3 & 5.97 & 5.61 & 1.6E-02 & 2.0E-02 & -1.28 \\
\hline Birc5 & 4.69 & 4.41 & 3.9E-02 & 4.7E-02 & -1.21 \\
\hline Bnip2 & 5.62 & 4.91 & 2.0E-02 & 3.3E-02 & -1.64 \\
\hline Bnip3 & 4.28 & 4.28 & 5.1E-02 & 5.1E-02 & 1.00 \\
\hline Bnip3I & 4.32 & 4.22 & 5.0E-02 & $5.4 \mathrm{E}-02$ & -1.08 \\
\hline Bok & 3.95 & 3.14 & $6.5 \mathrm{E}-02$ & $1.1 \mathrm{E}-01$ & -1.75 \\
\hline Card10 & 4.75 & 3.72 & 3.7E-02 & 7.6E-02 & -2.04 \\
\hline Casp1 & 6.88 & 6.15 & 8.5E-03 & 1.4E-02 & -1.65 \\
\hline Casp12 & 5.39 & 4.98 & 2.4E-02 & 3.2E-02 & -1.33 \\
\hline Casp14 & 3.88 & 2.77 & 6.8E-02 & 1.5E-01 & -2.16 \\
\hline Casp2 & 5.44 & 4.84 & 2.3E-02 & 3.5E-02 & -1.52 \\
\hline Casp3 & 4.37 & 3.32 & 4.8E-02 & $1.0 \mathrm{E}-01$ & -2.06 \\
\hline Casp4 & 7.07 & 7.17 & $7.4 \mathrm{E}-03$ & 6.9E-03 & 1.07 \\
\hline Casp6 & 6.61 & 6.12 & 1.0E-02 & 1.4E-02 & -1.41 \\
\hline Casp7 & 5.46 & 4.70 & 2.3E-02 & 3.8E-02 & -1.69 \\
\hline
\end{tabular}




\begin{tabular}{|c|c|c|c|c|c|}
\hline Casp8 & 6.43 & 6.08 & $1.2 \mathrm{E}-02$ & $1.5 \mathrm{E}-02$ & -1.27 \\
\hline Casp9 & 6.08 & 5.58 & 1.5E-02 & 2.1E-02 & -1.41 \\
\hline $\mathrm{Cd} 40$ & 5.96 & 6.16 & 1.6E-02 & 1.4E-02 & 1.14 \\
\hline Cd40lg & 7.03 & 6.33 & 7.6E-03 & $1.2 \mathrm{E}-02$ & -1.63 \\
\hline Cd70 & 6.44 & 6.54 & $1.2 \mathrm{E}-02$ & 1.1E-02 & 1.07 \\
\hline Cflar & 5.16 & 5.01 & 2.8E-02 & 3.1E-02 & -1.11 \\
\hline Cidea & 4.49 & 4.49 & 4.4E-02 & 4.4E-02 & -1.00 \\
\hline Cideb & 5.29 & 4.59 & $2.5 \mathrm{E}-02$ & $4.2 \mathrm{E}-02$ & -1.63 \\
\hline Cradd & 5.55 & 4.53 & 2.1E-02 & 4.3E-02 & -2.03 \\
\hline Dad1 & 5.14 & 4.17 & 2.8E-02 & 5.6E-02 & -1.97 \\
\hline Dapk1 & 6.64 & 6.21 & 1.0E-02 & 1.4E-02 & -1.35 \\
\hline Dffa & 7.25 & 7.41 & 6.6E-03 & 5.9E-03 & 1.11 \\
\hline Dffb & 6.58 & 6.06 & 1.0E-02 & 1.5E-02 & -1.44 \\
\hline Diablo & 6.04 & 5.83 & $1.5 \mathrm{E}-02$ & $1.8 \mathrm{E}-02$ & -1.16 \\
\hline Fadd & 4.50 & 3.24 & 4.4E-02 & 1.1E-01 & -2.39 \\
\hline Fas & 5.40 & 4.21 & $2.4 \mathrm{E}-02$ & $5.4 \mathrm{E}-02$ & -2.28 \\
\hline Fasl & 5.35 & 5.24 & $2.4 \mathrm{E}-02$ & 2.6E-02 & -1.08 \\
\hline Gadd45a & 7.40 & 7.12 & 5.9E-03 & 7.2E-03 & -1.22 \\
\hline $\lg 11 r$ & 3.87 & 2.61 & $6.8 \mathrm{E}-02$ & $1.6 \mathrm{E}-01$ & -2.40 \\
\hline II10 & 6.72 & 6.43 & $9.5 \mathrm{E}-03$ & $1.2 \mathrm{E}-02$ & -1.22 \\
\hline Lhx 4 & 5.86 & 5.77 & 1.7E-02 & $1.8 \mathrm{E}-02$ & -1.07 \\
\hline Ltbr & 4.72 & 3.68 & 3.8E-02 & $7.8 \mathrm{E}-02$ & -2.06 \\
\hline Mapk1 & 5.99 & 5.95 & 1.6E-02 & 1.6E-02 & -1.02 \\
\hline Mcl1 & 6.80 & 6.65 & 9.0E-03 & 1.0E-02 & -1.11 \\
\hline Naip1 & 6.44 & 6.59 & $1.2 \mathrm{E}-02$ & 1.0E-02 & 1.11 \\
\hline Naip2 & 6.38 & 5.83 & $1.2 \mathrm{E}-02$ & $1.8 \mathrm{E}-02$ & -1.47 \\
\hline Nfkb1 & 6.24 & 5.87 & 1.3E-02 & 1.7E-02 & -1.29 \\
\hline Nme5 & 5.52 & 5.23 & 2.2E-02 & 2.7E-02 & -1.23 \\
\hline Nod1 & 4.99 & 4.02 & 3.1E-02 & $6.2 \mathrm{E}-02$ & -1.97 \\
\hline Nol3 & 5.77 & 4.83 & $1.8 \mathrm{E}-02$ & 3.5E-02 & -1.92 \\
\hline Polb & 6.59 & 6.11 & 1.0E-02 & 1.5E-02 & -1.40 \\
\hline Prdx2 & 4.98 & 4.39 & 3.2E-02 & 4.8E-02 & -1.50 \\
\hline Pycard & 7.31 & 7.89 & 6.3E-03 & 4.2E-03 & 1.49 \\
\hline Ripk1 & 7.03 & 6.70 & 7.6E-03 & $9.6 \mathrm{E}-03$ & -1.27 \\
\hline Tnf & 6.46 & 6.86 & 1.1E-02 & 8.6E-03 & 1.32 \\
\hline Tnfrsf10b & 4.95 & 5.28 & 3.2E-02 & 2.6E-02 & 1.25 \\
\hline Tnfrsf11b & 6.29 & 5.67 & 1.3E-02 & 2.0E-02 & -1.54 \\
\hline Tnfrsf1a & 6.16 & 5.77 & 1.4E-02 & $1.8 \mathrm{E}-02$ & -1.31 \\
\hline Tnfsf10 & 5.43 & 4.57 & 2.3E-02 & 4.2E-02 & -1.82 \\
\hline Tnfsf12 & 6.51 & 6.23 & 1.1E-02 & 1.3E-02 & -1.21 \\
\hline Traf1 & 4.87 & 3.83 & $3.4 \mathrm{E}-02$ & 7.0E-02 & -2.06 \\
\hline
\end{tabular}




\begin{tabular}{|c|c|c|c|c|c|}
\hline Traf2 & 6.42 & 6.81 & 1.2E-02 & 8.9E-03 & 1.31 \\
\hline Traf3 & 6.73 & 6.41 & 9.4E-03 & 1.2E-02 & -1.25 \\
\hline Trp53 & 4.62 & 3.30 & 4.1E-02 & $1.0 \mathrm{E}-01$ & -2.50 \\
\hline Trp53bp2 & 6.66 & 6.55 & 9.9E-03 & 1.1E-02 & -1.08 \\
\hline Trp63 & 6.22 & 5.36 & 1.3E-02 & 2.4E-02 & -1.82 \\
\hline Trp73 & 6.78 & 6.63 & 9.1E-03 & 1.0E-02 & -1.11 \\
\hline Xiap & 7.01 & 6.67 & 7.8E-03 & $9.8 \mathrm{E}-03$ & -1.27 \\
\hline Actb & 4.62 & 4.17 & 4.1E-02 & 5.6E-02 & -1.37 \\
\hline $\mathrm{B} 2 \mathrm{~m}$ & 2.97 & 1.60 & 1.3E-01 & 3.3E-01 & -2.58 \\
\hline Gapdh & 0.00 & 0.00 & $1.0 \mathrm{E}+00$ & $1.0 \mathrm{E}+00$ & -1.00 \\
\hline Gusb & 5.31 & 4.47 & $2.5 \mathrm{E}-02$ & 4.5E-02 & -1.79 \\
\hline Hsp90ab1 & 2.84 & 1.73 & $1.4 \mathrm{E}-01$ & 3.0E-01 & -2.16 \\
\hline
\end{tabular}




\section{Vita}

Kinsey A. Hilliard was born to Mark and Denise Hilliard on December 1, 1991 in Olney, Illinois. She was raised in Fairfield, Illinois and is the younger sister of Christopher Hilliard. Kinsey graduated from Fairfield Community High School in 2010 and did her undergraduate work at Blackburn College in Carlinville, Illinois, where she received her Bachelor of Arts degree in Biology in 2014. She was accepted into the Molecular Pathogenesis and Therapeutics graduate program at the University of Missouri in 2014, training under the supervision of Dr. Charles R. Brown. Kinsey received her Ph.D. in Microbiology in May 2019. 